\title{
DEVELOPMENT OF A SINGLE-STAGE IMPLOSION-DRIVEN HYPERVELOCITY LAUNCHER
}

\author{
Daniel Szirti \\ Masters of Engineering \\ Department of Mechanical Engineering
}

\author{
McGill University \\ Montreal,Quebec
}

May 6, 2008

\begin{abstract}
A thesis submitted to the
Faculty of Graduate Studies and Research

In partial fulfillment of the requirements of the degree

Master of Engineering

(C)Daniel Szirti

June 2008
\end{abstract}


Library and

Archives Canada

Published Heritage

Branch

395 Wellington Street

Ottawa ON K1A 0N4

Canada
Bibliothèque et

Archives Canada

Direction du

Patrimoine de l'édition

395 , rue Wellington

Ottawa ON K1A ON4

Canada

Your file Votre référence

ISBN: 978-0-494-51476-4

Our file Notre référence

ISBN: 978-0-494-51476-4

NOTICE:

The author has granted a nonexclusive license allowing Library and Archives Canada to reproduce, publish, archive, preserve, conserve, communicate to the public by telecommunication or on the Internet, loan, distribute and sell theses worldwide, for commercial or noncommercial purposes, in microform, paper, electronic and/or any other formats.

The author retains copyright ownership and moral rights in this thesis. Neither the thesis nor substantial extracts from it may be printed or otherwise reproduced without the author's permission.
AVIS:

L'auteur a accordé une licence non exclusive permettant à la Bibliothèque et Archives Canada de reproduire, publier, archiver, sauvegarder, conserver, transmettre au public par télécommunication ou par l'Internet, prêter, distribuer et vendre des thèses partout dans le monde, à des fins commerciales ou autres, sur support microforme, papier, électronique et/ou autres formats.

L'auteur conserve la propriété du droit d'auteur et des droits moraux qui protège cette thèse. $\mathrm{Ni}$ la thèse ni des extraits substantiels de celle-ci ne doivent être imprimés ou autrement reproduits sans son autorisation.
In compliance with the Canadian

Privacy Act some supporting forms may have been removed from this thesis.

While these forms may be included in the document page count, their removal does not represent any loss of content from the thesis.
Conformément à la loi canadienne sur la protection de la vie privée, quelques formulaires secondaires ont été enlevés de cette thèse.

Bien que ces formulaires aient inclus dans la pagination, il n'y aura aucun contenu manquant.

\section{Canada}




\begin{abstract}
The present study deals with the development of a single-stage implosion-driven hypervelocity launcher. A thin-walled tube filled with helium surrounded by explosives acts as a driver for the launcher. Implosion of the tube drives a strong shock that reflects back and forth between the projectile and the implosion pinch, generating very high temperatures and pressures. Simple analytic models were used to approximate the performance of the pump tube and its use as a driver for a launcher. Experiments to evaluate the implosion dynamics and performance of the pump tube were carried out, and implosion-driven launcher experiments demonstrated muzzle velocities above $4 \mathrm{~km} / \mathrm{s}$ with 5 -mm-diameter aluminum projectiles. Projectile integrity was verified by high-speed photography. Disagreement of experimental data with the analytical models of performance is mostly due to failure to seal the chamber of the launcher, resulting in loss of driver gas, and pump tube expansion, which weakens the precursor shock.
\end{abstract}




\begin{abstract}
ABRÉGÉ
Cette étude concerne le développement d'un lanceur à hyper-vitesse à implosion à un étage. Un tube aux parois minces rempli d'hélium et entouré d'explosifs actionne le lanceur. L'implosion du tube génère une onde de choc qui se réfléchi entre le projectile et le pincement dû à l'implosion, ce qui produit de très hautes pressions et températures. Des modèles analytiques simples furent utilisés pour estimer la performance du tube de compression et son utilité en tant qu'actionneur pour le lanceur. La performance du tube de compression fût vérifiée expérimentalement, et des essais expérimentaux du lanceur ont démontré des vitesses de bouche de plus de $4 \mathrm{~km} / \mathrm{s}$ pour des projectiles de $5 \mathrm{~mm}$ de diamètre. L'intégrité du projectile fût vérifiée par photographie à haute fréquence. L'incapacité de sceller la chambre du lanceur, ce qui cause la perte de gaz, et l'expansion du tube de compression, ce qui atténue l'onde de choc, sont les principales causes de la différence entre les résultats expérimentaux et ceux des modèles analytiques.
\end{abstract}




\section{ACKNOWLEDGEMENTS}

Many are those who contributed to this research project, and I am indebted to all of them for helping me reach the objectives I had set for myself and for my research. First and foremost I would like to thank my supervisor Andrew J. Higgins for his guidance, his support, and his profound enthusiasm towards academic research and this project in particular. My thanks go to all the members of the SWPG at McGill University for their opinions, ideas, and criticism throughout this project. Special thanks go to Patrick Batchelor and Jason Loiseau for the inspiration they have given me and for all the work they put into the design and construction of the launcher. I thank Dr. Nicolas Ponchaut for the use of his one-dimensional Eulerian solver, the time he spent adapting it to the purposes of this project, and for instructing me in its use. I also thank Vincent Tanguay of DRDC Valcartier for his continued interest and work on the launcher. Many of the McGill technical staff contributed to the machining and construction of the launcher, namely Gary Savard, John Boisvert, Ray Lemay, Tony Micozzi, Roy Westgate, and Steve Kakani; my thanks go to them. I also extend my thanks to Aleks Labuda who also helped with the machining of launcher parts. I would like to thank Darius Nikanpour and Dean Sangiorgi of the Canadian Space Agency for their encouragement and their faith my research group's capabilities. I am grateful for the funding provided by the Canadian Space Agency and the Department of National Defense toward the development of the launcher. Finally, I thank my family and friends for their love, support and understanding. 


\section{TABLE OF CONTENTS}

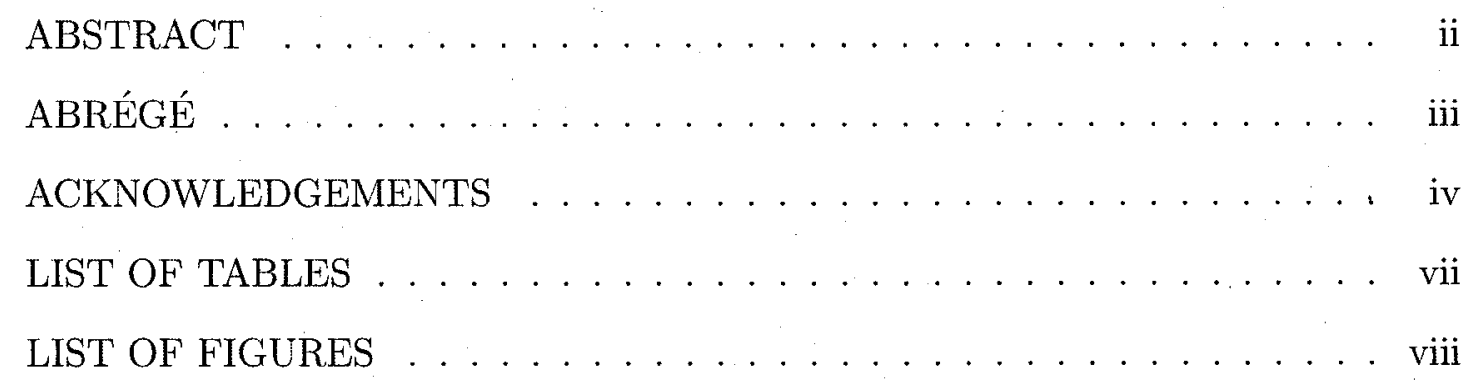

1 Introduction . . . . . . . . . . . . . . . . . 1

1.1 The Linear Explosive Driver . . . . . . . . . . . . . . . . . . . 2

1.1.1 Ideal Operation . . . . . . . . . . . . . . . . . 2

1.1 .2 Non-Ideal Behaviour . . . . . . . . . . . . . . . . 4

1.2 The Linear Explosive Launcher . . . . . . . . . . . . . . . . . . . 13

1.2.1 Ideal Light Gas Gun Theory . . . . . . . . . . . . . . . 13

1.2.2 The Linear Explosive Driver Used as a Hypervelocity

Launcher . . . . . . . . . . . . . . 16

1.3 Present Study . . . . . . . . . . . . . . . . . . . 21

2 Expanding Tube Model . . . . . . . . . . . . . . . 22

2.1 Derivation of the Model . . . . . . . . . . . . . . . 23

2.1.1 Pump Tube Motion . . . . . . . . . . . . . . . . . 23

2.1 .2 Outer Tube Motion . . . . . . . . . . . . . . . . . . 28

2.1 .3 Driver Gas Pressure . . . . . . . . . . . . . . . . . . . . . . 29

2.1 .4 Explosive Layer Pressure . . . . . . . . . . . . . 30

2.1 .5 Summary . . . . . . . . . . . . . . . . 37

2.2 Model Results . . . . . . . . . . . . . . . . . . . 38

2.2.1 Calculated Expansion Histories . . . . . . . . . . . . . . . . . . . . . . . 38

2.2 .2 Standoff Calculation . . . . . . . . . . . . . 43

$3 \quad$ Driver Experiments . . . . . . . . . . . . . . . . . . . . . . 48

$3.1 \quad 0.64 \mathrm{~cm}$ Outer Diameter Pump Tube Series . . . . . . . . . . . . 48

3.1 .1 Experimental Setup . . . . . . . . . . . . . . . . . 48

3.1 .2 Results ..................... 50 
3.1.3 Comparison with Expanding Tube Model . . . . . . . . . . 56

$3.2 \quad 0.95 \mathrm{~cm}$ Outer Diameter Pump Tube Series . . . . . . . . . . . . . . 57

$3.3 \quad 1.27 \mathrm{~cm}$ Outer Diameter Pump Tube Series . . . . . . . . . . . 58

3.3 .1 Shot D014 . . . . . . . . . . . . . . . . . . 59

3.3 .2 Shot D015 . . . . . . . . . . . . . . 61

3.3 .3 Shot D016 . . . . . . . . . . . . . . . 63

3.3 .4 Shot D017 . . . . . . . . . . . . . 64

3.3 .5 Shot D018 . . . . . . . . . . . . . . . . . 65 65

3.3.6 Shots Using Detasheet Explosives . . . . . . . . . . 67

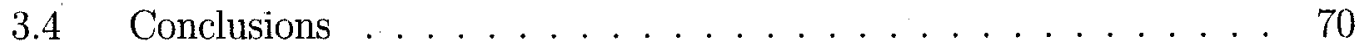

4 Theoretical Internal Ballistics . . . . . . . . . . . . . . 71

4.1 Method of Characteristics . . . . . . . . . . . . 72

4.2 1-D Euler Code . . . . . . . . . . . . . . . . . . . . 76

4.3 Validation of the Numerical Codes . . . . . . . . . . . . 77

4.3.1 Comparison of Both Codes With Each Other . . . . . . . 77

4.3.2 Comparison of Both Codes With Theory . . . . . . . . 79

4.4 Numerical Modeling of the Launcher . . . . . . . . . . . . . . 80

$5 \quad$ Launcher Experiments . . . . . . . . . . . . . . . . . . 93

$5.1 \quad$ Launcher Design . . . . . . . . . . . . . . . . . . 93

5.1 .1 Launcher Chamber . . . . . . . . . . . . . 93

5.1 .2 Launch Tube . . . . . . . . . . . . . . . . . . . . 97

5.1 .3 Diagnostics . . . . . . . . . . . . . . . . . . . . . . . . . . . . . . . . .

5.2 Experimental Launcher Performance . . . . . . . . . . . . . 101

5.2 .1 Compression Ratio Variation . . . . . . . . . . . . . 102

5.2 .2 Initial Fill Pressure Variation . . . . . . . . . . . . 105

6 Conclusions . . . . . . . . . . . . . . . . . 110

Appendix A - Expanding Tube Model Algorithm . . . . . . . . . . . . . . . . 112

Appendix B - Final Launcher Part Drawings . . . . . . . . . . . . 116

References . . . . . . . . . . . . . . . . . . 118 
3-1 Summary of driver experiments using $0.64 \mathrm{~cm}$ outer diameter pump tubes ............................ 51

3-2 Summary of driver experiments using $0.95 \mathrm{~cm}$ outer diameter pump tubes ........................... 58

3-3 Summary of driver experiments using detasheet explosives . . . . . . 68

5-1 Summary of launcher experiments using $0.95 \mathrm{~cm}$ outer diameter, $1.65 \mathrm{~mm}$ wall thickness pump tubes . . . . . . . . . . . . 94

5-2 Summary of initial launcher experiments using tamped $1.27 \mathrm{~cm}$ outer diameter, $0.9 \mathrm{~mm}$ wall thickness pump tubes . . . . . . . . . 95

5-3 Summary of launcher experiments testing compression ratio variation . 106 5-4 Summary of launcher experiments testing initial fill pressure variation 108 
1-1 Operation of the linear explosive driver . . . . . . . . . . . 3

1-2 Metal jet formation with a cylindrical liner . . . . . . . . . 9

1-3 Typical launcher setup . . . . . . . . . . . . . . . . . 13

1-4 Operation of the implosion-driven launcher . . . . . . . . . . 17

1-5 Auxiliary pump schematic . . . . . . . . . . . . . 21

$2-1$ Typical driver cross sections . . . . . . . . . . . . . . 23

$2-2$ Force balance on pump tube segment . . . . . . . . . . . 24

2-3 Position-time graph showing expanding pump tube and generated acoustics for an infinite explosive layer . . . . . . . . . . . 32

2-4 Position-time graph showing expanding pump tube and acoustic reverberations . . . . . . . . . . . . . . 33

2-5 Zoom of the position-time graph of the expanding tube showing one time-step ........................ 34

2-6 Expansion histories for various pressures . . . . . . . . . . 40

2-7 Parameter maps . . . . . . . . . . . . . . . . . . 42

2-8 Volume of driver gas contained in the pump tube . . . . . . . . . 44

2-9 Converting time to distance . . . . . . . . . . . . . 45

2-10 Calculated standoff distances at $52 \mathrm{~cm}$ from the initial collapse point for a $3.175 \mathrm{~mm}$ radius pump tube as a function of fill pressure . . . 46

3-1 Schematic of the experimental apparatus used in the pressure variation series . . . . . . . . . . . . . . . . . . . . 49

3-2 Position-time graph for 27.2 atm initial fill pressure . . . . . . . 50 
3-3 Precursor shock velocity and standoff $v s$ initial fill pressure for a $0.64 \mathrm{~cm}$ outer diameter pump tube . . . . . . . . . . . 52

3-4 Photograph of cross-sectioned recovered pump tubes showing incom-

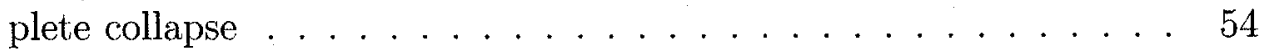

3-5 Photograph of recovered pump tubes showing rupturing along the length of the tubes for the higher pressure shots . . . . . . . 55

3-6 Photograph of recovered pump tubes showing that total recovered length of the tube tends to decrease as we increase initial fill pressure 55

3-7 Calculated standoff distances for a $3.175 \mathrm{~mm}$ radius pump tube as a function of fill pressure, compared to experimental results . . . . 56

3-8 Typical setup for $1.27 \mathrm{~cm}$ outer diameter driver shot . . . . . . . . . 59

3-9 Photograph of the recovered pump tube for shot D014 showing a complete collapse initially and rupturing along most of its length . 61

3-10 Experimental setup for a $1.8 \mathrm{~m}$ long driver shot . . . . . . . . . 62

3-11 $x-t$ graph for a $1.8 \mathrm{~m}$ long driver with 20.4 atm initial fill pressure . 64

3-12x-t graph for a $1.8 \mathrm{~m}$ long driver with 68 atm initial fill pressure . . 66

3-13 $x-t$ graph for an untamped driver with 20.4 atm initial fill pressure $\quad 67$

3-14 Experimental setup for driver shots using detasheet explosive . . . . . 69

3-15 Photograph of the recovered pump tube fragments for shots D019

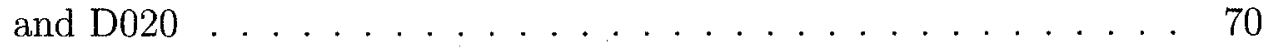

4-1 Diagram of the test case . . . . . . . . . . . . . . 74

4-2 Sample $x-t$ graph for the method of characteristics code . . . . . 75

4-3 Sample computational $x-t$ schlieren from the Euler code . . . . : 77

4-4 Comparison of results obtained from both codes for a sample test case 78

4-5 The unchambered, finite chamber length, PPIG launcher . . . . . . 79

4-6 Performance of an unchambered, finite chamber length, PPIG launcher 81

4-7 Performance of a finite chamber length, chambered, PPIG launcher $\left(D_{0} / D_{1}=1.778, \gamma=1.4, G / M=1\right) \ldots \ldots . \ldots 81$

$4-8$ Definitions of launcher dimensions . . . . . . . . . . . . . . 83 
4-9 Computational $x-t$ schlieren from the Euler code for different compression ratios . . . . . . . . . . . . . . 84

4-10 Projectile velocity profiles as a function of distance traveled down the launch tube for various compression ratios . . . . . . . . 86

4-11 Projectile velocity as a function of compression ratio for various initial fill pressures . . . . . . . . . . . . . . . 87

4-12 Computational $x-t$ and $x-v$ graphs from the Euler code for different retreating piston velocities . . . . . . . . . . . . . . 89

4-13 Computational $x-t$ and $x-v$ graphs from the Euler code for different compression ratios . . . . . . . . . . . . . . 91

4-14 Projectile velocity as a function of compression ratio for various initial fill pressures. The piston reverses direction once it reaches the chamber to simulate its destruction. . . . . . . . . . . . . 92

5-1 Experimental launcher $\operatorname{design} \ldots \ldots . . \ldots 103$

5-2 Experimental launcher setup, showing facilities and diagnostics . . . 104

5-3 Picture of launchers, before and after firing . . . . . . . . . . . . . . . 104

5-4 Projectile velocity for compression ratios from 6 to 16 for an initial fill pressure of $20 \mathrm{~atm} \ldots \ldots \ldots \ldots$. . . . . . . . . 107

5-5 Projectile velocity for initial fill pressures from 20 to $50 \mathrm{~atm}$ for a compression ratio of $11.5 \ldots \ldots \ldots \ldots$. . . . . . . . 107

5-6 Pictures of breech cones after firing for various initial fill pressures . . 109 


\section{CHAPTER 1 \\ Introduction.}

Hypervelocity launchers are devices which accelerate projectiles to very high velocities, typically several kilometers per second. These devices find applications in many diverse fields, mostly as laboratory tools for generating high velocity impacts. While there are many ways to accelerate projectiles to hypervelocities, the current methods have more or less reached their maximum potential in terms of projectile size and terminal velocity. For example, modified light gas guns can at best generate velocities of around $10 \mathrm{~km} / \mathrm{s}$ for projectile masses in the range of $1 \mathrm{~g}$ to $10 \mathrm{~g}$. If we are to achieve greater velocities, new types of launchers must be developed.

A novel concept was investigated by the Physics International company under funding from NASA in the 1960's. Their launcher incorporated two sections: a linear explosive driver section and a gun section. The driver section compresses a light gas to high temperature and pressure, and in this regard resembles the concept of the light gas gun. However; whereas light gas guns use a piston to compress the gas, the Physics International driver used a detonation wave to progressively implode the driver tube, earning it the name of "implosion-driven launcher." The resulting hot high-pressure gas is delivered to the gun section, which uses it as a propellant to accelerate the projectile. Their launcher also used a second stage, where the launch tube was imploded behind the projectile as well. The resulting two-stage launcher demonstrated maximum velocities of around $14 \mathrm{~km} / \mathrm{s}$ for a 2 g projectile, a record velocity for this mass at the time. 
The majority of the work carried out on this concept can be found in four NASA contract reports written by the Physics International company $[4,10,17,1]$. The present study is aimed at better understanding and improving the single-stage launcher exclusively.

\subsection{The Linear Explosive Driver}

\subsubsection{Ideal Operation}

A linear explosive driver converts some of the chemical energy of an explosive into internal and kinetic energy of a gas to obtain higher energy densities than conventionally possible. This is done by using the high detonation pressure of the explosive to implode a thin-walled tube (called a "liner" or "pressure tube" or "pump tube") filled with a light gas (called the "driver gas"). The implosion pinch travels down the tube at the detonation velocity of the explosive and acts as a cone-shaped virtual piston which drives a strong shock in the driver gas in front of it. See figure 11 for a sketch of the operation of this device.

Since the objective of the linear explosive driver is to generate a column of high pressure and temperature gas, it is important to know the state of the gas at the end of the driver's operation. For a perfect gas, the Mach number $M_{s}$ of a shock wave propagating into an initially quiescent gas can be obtained through the following relation:

$$
\frac{U_{p}}{c_{0}}=\frac{2}{\gamma+1}\left(\frac{M_{s}^{2}-1}{M_{s}}\right)
$$

where $U_{p}$ is the velocity of the piston, $c_{0}$ is the initial sound speed in the gas, $\gamma$ is the ratio of the specific heats of the gas, and $M_{s}$ is the Mach number at which the shock wave is traveling. In the limit of strong shocks, the shock velocity is about $(\gamma+1) / 2$ times the piston velocity. For example, if we use nitromethane as an explosive (detonation velocity $\approx 6 \mathrm{~km} / \mathrm{s}$ ) and helium as a driver gas $(\gamma=1.666)$, we 


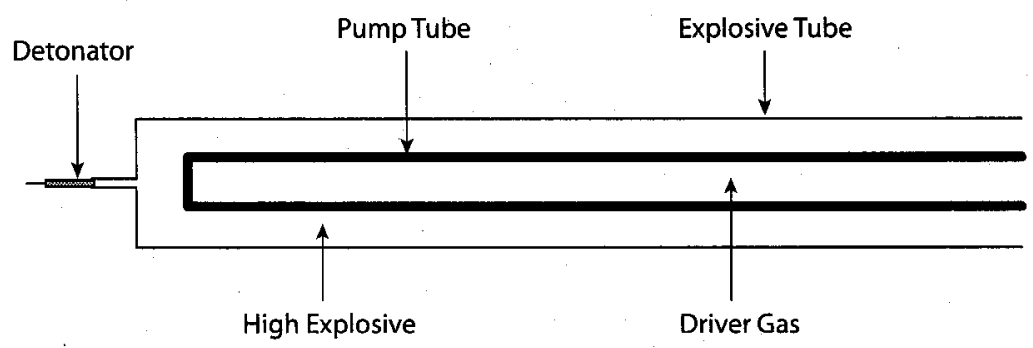

(a) Driver before detonation

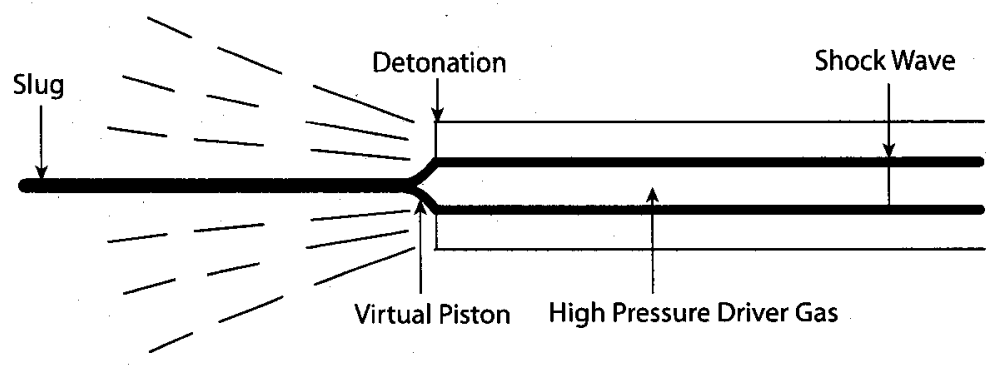

(b) Driver during operation

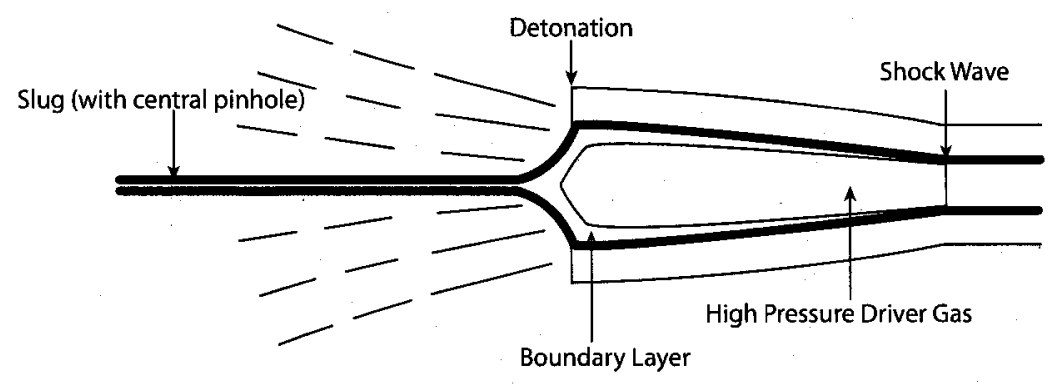

(c) Driver showing non-ideal effects

Figure 1-1: Operation of the linear explosive driver 
would expect an $8.1 \mathrm{~km} / \mathrm{s}$ shock wave. As the piston moves further down the tube, the separation between it and the shock increases, and ideally is only limited by the length of the tube. The pressure and temperature behind the shock can be easily obtained through normal shock relations:

$$
\begin{gathered}
\frac{p_{s}}{p_{0}}=\frac{2 \gamma M_{s}^{2}-(\gamma-1)}{\gamma+1} \\
\frac{T_{s}}{T_{0}}=\frac{\left[2 \gamma M_{s}^{2}-(\gamma-1)\right]\left[2+(\gamma-1) M_{s}^{2}\right]}{(\gamma+1)^{2} M_{s}^{2}}
\end{gathered}
$$

where the subscripts 0 and $s$ denote the initial and shocked states. The speed of sound behind the shock $c_{s}$ can be obtained through the following relation:

$$
c_{s}=\sqrt{\gamma R T_{s}}
$$

where $R$ is the specific gas constant. For the example given above, the Mach number of the shock is about 8.0. This gives a pressure ratio across the shock of about 80 and a temperature ratio of about 21. For a driver filled with helium at 20 atm and $300 \mathrm{~K}$, this means the pressure, temperature, and speed of sound behind the shock are about $1600 \mathrm{~atm}, 6300 \mathrm{~K}$, and $4.6 \mathrm{~km} / \mathrm{s}$.

\subsubsection{Non-Ideal Behaviour}

Many experiments were carried out by Moore [10] to observe the performance of the linear explosive driver over a wide range of parameters. Departure from ideal behaviour can be mostly explained by three mechanisms: "(1) radial expansion of the pressure tube behind the shock wave, (2) the effect of boundary layer growth behind the shock wave, and (3) formation of a metal or metal-gas jet by the collapsing pressure tube" [10]. These effects are interrelated, but their individual effects may still be determined. 


\section{Radial Expansion}

If the shocked gas pressure exceeds the yield strength of the tube, the tube will expand radially. For short driver lengths this effect is negligible, but as the shock gets further ahead of the detonation, this yielding will allow the tube to expand and attenuate the precursor shock. Experiments show that as the initial fill pressure of the gas increases, the velocity of the shock decreases [10]. Eventually this expansion can cause the tube to burst and vent out its driver gas. The wall thickness of the tube may be increased to avoid expansion and bursting, but at higher pressures the thickness needed renders the tube difficult to implode. Another strategy is to surround the explosives with a thick-walled pressure vessel called a "tamper." Once the pump tube is exposed to shock pressure, it will expand and compress the explosive layer. However, the explosive layer is now confined by a thick-walled tube. The pressure in the explosive layer will then increase until either it has reached the shock pressure inside the pump tube or the tamper yields. If the tamper is thick enough not to yield from the shock pressure in the pump tube, pressures will equilibrate and the expansion of the pump tube will be halted. Even if its yield strength is surpassed, the tamper still provides inertial confinement, slowing the expansion of the pump tube. The tamper also serves the extra purpose of delaying the expansion of the detonated explosives outward and focusing its pressure inward, towards the pump tube, allowing thinner layers of explosives to be used and making it possible to obtain longer lengths of shocked driver gas.

Watson [17] performed experiments to evaluate how the pressure of the shocked driver gas behind the shock wave is influenced by pump tube expansion. These experiments revealed that the pressure inside the pump tube is relatively constant despite radial expansion. Watson states that "the increased volume of gas caused by 
expansion appears to be compensated by an adjustment in length of the column of shocked gas" [17]. This phenomenon will be discussed in section 2.1.3.

\section{Boundary Layer}

Experimental attempts by Moore [10] to produce an arbitrarily long column of shocked gas revealed that there was a maximum attainable length for a given configuration. The length increases at first, but eventually reaches a maximum value as the shock velocity gradually decays down to the detonation velocity of the explosive. This type of experiment used $3.8 \mathrm{~m}$ long pump tubes. Examination of recovered pump tubes revealed that it was collapsed perfectly along the first $1 / 5$ of its length, "then the internal diameter gradually began to increase until it reached a constant value that was about one-half of what it was prior to collapse" [10]. It is believed this is due to the development of a boundary layer along the inside of the liner.

This phenomenon is similar to the one observed in shock tubes. In a shock tube, shock waves are generated by having a high-pressure gas expand into a lower pressure gas. The contact surface between both gases acts as a piston. In the ideal case, both the contact surface and the shock move at constant speed (but not at the same speed; their speeds are related by equation 1.1). However, due to viscous effects, the relative motion of the shocked gas against the tube wall causes a boundary layer to develop behind the precursor shock. The boundary layer effectively removes mass from the region between the shock wave and the contact surface, which causes a deceleration of the shock and an acceleration of the contact surface, and prevents an infinite separation distance between both. This separation distance reaches a maximum once both the shock and contact surface move at the same speed; this 
happens when the mass entering through the shock is equal to the mass exiting through the boundary layer.

In a linear explosive driver, a boundary layer develops as in the shock tube. The difference here is that the piston speed is constant since it is determined by the detonation velocity of the explosive. The gas inside the boundary layer "receives little axial acceleration and is trapped by the collapsing tube" [16]. This results in a loss of shocked driver gas, which attenuates the precursor shock until the mass of gas entering the shock equals the mass of gas lost to the collapsing liner. At this point, a steady state is reached where the shock travels at the detonation velocity of the explosive and the length of shocked gas attains a maximum value.

Mirels [9] performed an analysis to estimate shock tube test time limitations due to turbulent boundary layers. Continuity and turbulent boundary layer theory are used, and the development of the boundary layer is allowed to influence the freestream conditions. The result is an estimate of both the final separation distance between the shock and the contact surface and of the variation of this separation distance with respect to the distance traveled by the shock. This analysis, however, neglects the communication time between the piston and the shock front. In [16], this communication time was incorporated into a one dimensional computer model and "was treated in finite time steps involving first a delay for the boundary layer to build up at a fixed position, and then a second interval for rarefactions to overtake the shock wave" [16]. Results obtained by incorporating this communication time yielded much better results when compared to experiments $[16,17]$.

\section{Jet Formation}

Under certain conditions, shock velocities higher than theoretical were obtained by Moore [10]. Implosively collapsing tubes is a known way of obtaining high-velocity 
metal jets, therefore the presence of such a jet was postulated as the reason of such unusually high velocities. At first, a high shock velocity may seem like a good thing, but as Waldron [16] states, if a jet is present, it will initially increase the shock velocity and distance from the shock to the detonation, but will mix liner material to the driver gas. This is disadvantageous in launcher applications since, as will be discussed in section 1.2, the molecular weight of the driver gas has to be as low as possible. In order to verify the presence of such a jet and whether this jet would mix with the driver gas, Watson [17] carried out experiments for conditions known to produce higher than theoretical shock velocities. Pulsed x-ray photographs taken by Watson [17] clearly show a metal jet severely mixed with the driver gas. The need to prevent liner jetting is clear.

The classical theory for jet formation with conical and wedge shaped liners is presented in [2]. It is assumed that the liner behaves as an inviscid fluid and that, after the initial impulse the walls receive from the detonation wave, "the pressure on all sides of the liner quickly equalizes and the walls continue to collapse inward with no appreciable change in velocity" [2]. The ensuing equations are derived from geometry and the condition of steady state. Figure 1-2 shows a jet being formed with a cylindrical liner. The angle $\beta$ is the collapse angle, and the angle $\theta$ is the turning angle (the angle through which the tube is turned by the detonation). For cylindrical liners, both angles have the same value. The mass of the jet and the slug are:

$$
m_{j}=\frac{m}{2}(1-\cos \beta)
$$

and

$$
m_{s}=\frac{m}{2}(1+\cos \beta)
$$




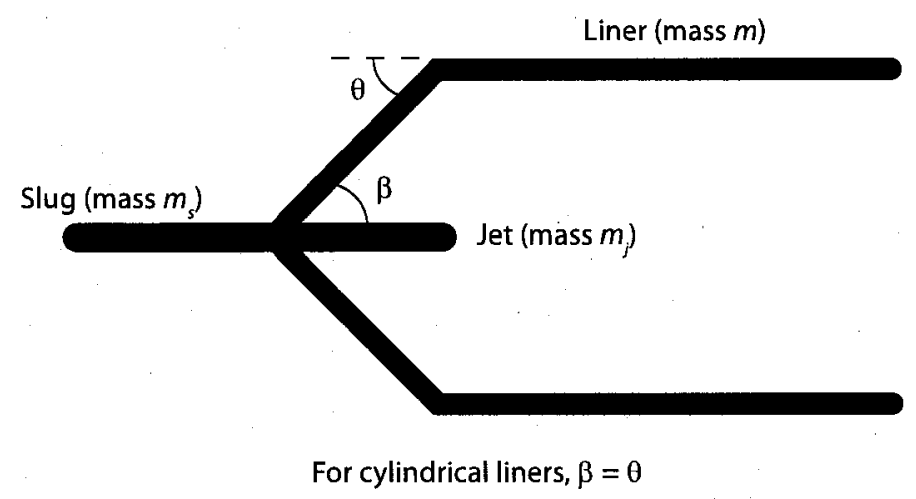

Figure 1-2: Metal jet formation with a cylindrical liner

where the subscripts $j$ and $s$ represent the jet and the slug, and $m$ is the total mass per unit length of the liner, so $m=m_{j}+m_{s}$. The slug is the collapsed remains of the liner. For cylindrical liners, the velocity of the jet is found to be $V=2 U_{d}$, where $U_{d}$ is the detonation velocity of the explosive. Experiments in a near vacuum performed by Koski [8] showed that there is a "fast jet" formed when collapsing cylindrical liners as well as the "penetrating jet" which travels at the predicted velocity of twice the detonation velocity. The fast jet goes faster than the penetrating jet and has significantly less mass. However, these fast jets are observed only for very low pressures and are not present in non-evacuated environments. Since linear explosive drivers are usually filled with high-pressure driver gas, these fast jets are not expected to be present.

When the detonation collapses the liner, a shock wave forms in the liner which turns the liner material into the axial direction. However, this shock has a maximum turning angle, $\theta_{\max }$. If the inner collapse angle is less than the turning angle, there will be no jet formed. If the inner collapse angle is greater than the maximum turning angle, a detached shock forms and part of the liner forms a jet. It is therefore desirable to have a high maximum turning angle and a low collapse angle. The 
turning angle depends on the Mach number of the "liner flow", which is approximately the detonation velocity divided by the liner sound speed. Therefore a high detonation velocity and a low liner sound speed would help to prevent jet formation. The collapse angle depends on the internal gas pressure, the inner radius, the thickness of the liner, the material properties of the liner, the thickness and properties (detonation velocity and energy density) of the explosive, and the presence of a tamper. Numerical simulations were done in [16] to approximate collapse profiles for different conditions. These simulations are not exact representations of the collapse behaviour, but show the effect of these various parameters. Results indicate that a higher shock pressure inside the liner and a thicker liner will diminish the collapse angle. However, since the mass of the jet is a fraction of the liner mass, using a thinner liner will also minimize the mass of the jet.

A simple analysis is made in [17] on the jetting phenomenon. It is based completely on the conservation laws and the condition of steady state, and it considers losses in kinetic energy from irreversible processes. The following equation for estimating the mass of the jet is obtained:

$$
\frac{m_{j}}{m}=\frac{\frac{\theta^{2}}{2}-\frac{4}{\sqrt{3}} \frac{Y \theta}{\rho U_{d}^{2}}}{2}
$$

where $Y$ is the Von Mises yield strength and $\rho$ is the liner density. For example, for a steel liner using nitromethane as the explosive, assuming a $45^{\circ}$ collapse angle, the percentage of liner mass going into the jet is $15 \%$. Moore concludes that small irreversible processes, such as plastic work of deformation and shock heating, are important and tend to prevent jetting, as do the internal pressure and the growth of a boundary layer [10]. This is consistent with experimental findings since jetting never seemed a problem in Watson's [17] launcher tests. 
Crosby and Gill [4] attempted to reduce the explosive loading such that it only barely collapses the tube; this should reduce the collapse angle to its minimum value and no metal jet should be formed. High initial driver gas fill pressures should also diminish the collapse angle and inhibit jet formation even for higher explosive loadings. However, experiments done by Moore [10] showed that varying the explosive-to-pressure-tube mass ratio and detonation velocity had no significant effect on performance.

As reported by Waldron [16] and Moore [10], it is also possible to have combined effects: if in the initial stages there is a liner jet of small mass, as the boundary layer thickness increases, the jetting will stop and boundary layer gas will be swallowed by the collapsing liner at later times: So in this case, there would be jetting effects and negligible boundary layer effects in the beginning of the driver operation, and the reverse at later times. This shows that different effects can be present at different times of the driver operation and can interact with each other.

\section{Collapse Process}

As we increase the initial fill pressure of the pump tube, we expect the tube to be more difficult to implode properly. Experiments done by Crosby and Gill [4] revealed that higher pressure shots do require more explosives to properly collapse. Also, if the shock wave reflects off the sealed end of the tube and interacts with the virtual piston, it is possible the reflected shock pressure is high enough to disturb the piston. However, Crosby and Gill determined experimentally that the reflected pressure has little or no influence on the incoming piston. Increasing the explosive loading would resolve any effect the initial fill pressure and the reflected pressure may have on driver operation. 
Kinelovskii studied the initial stage of collapse for tubes of various materials and dimensions. He proposed an analytic model to describe the motion of the inner and outer wall surfaces of the collapsing tube. This model is valid up until the point where the inner surface of the tube reaches $20 \%-30 \%$ of its initial value and it assumes that the pipe material is an ideal incompressible fluid. The tube is treated as a series of elementary independent transverse rings, and once the detonation reaches them, they are activated and behave as cylindrical pistons upon which the adiabatically expanding detonation products act. The analytic, nondimensional expressions are presented in [6] for the case of a tube surrounded by an infinitely thick layer of explosives; the case of a finite layer is considered in [7]. Although these expressions are simple to use and are shown to correlate fairly well with experiments, it is important in the present study to approximate the entire collapse profile, of particular use are determining the collapse angle and the conditions for obtaining a complete pinch. Since these cannot be determined by this analytic model, it was not used in this study.

\section{Ionization of the Driver Gas}

Typically extremely high pressures and temperatures are achieved during the launcher operation, of the order that may influence the driver gas properties. Ionization may occur in helium according to the following reaction:

$$
H e \leftrightarrow H e^{+}+e^{-}
$$

However, Watson showed using the Saha equation of state, that helium behaves as an ideal gas for very high pressures and temperatures and that these dissociation effects are negligible [17]. 


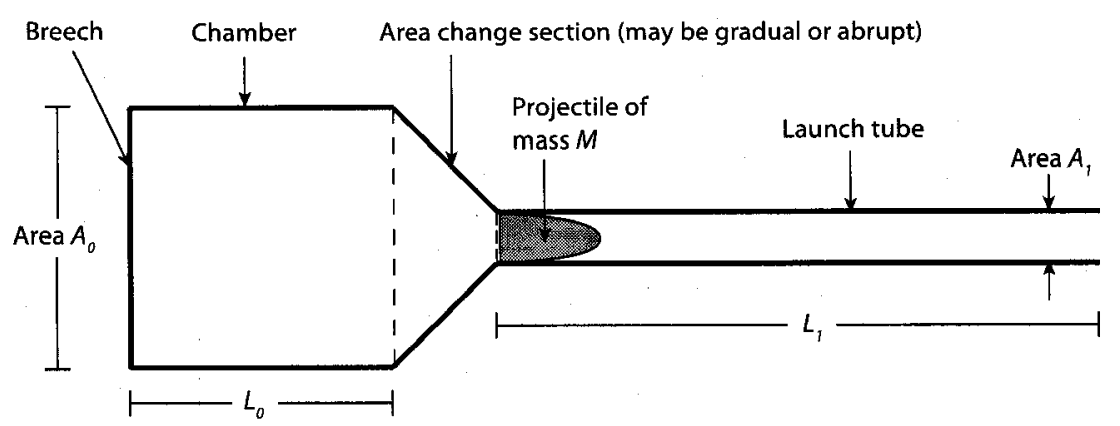

Figure 1-3: Typical launcher setup

\subsection{The Linear Explosive Launcher}

\subsubsection{Ideal Light Gas Gun Theory}

Before discussing how the linear explosive driver can be used for launcher applications, it is good to review the theory of high speed guns. A thorough treatment of the subject is given by Seigel [14]. It is assumed here that friction in the launch tube (or barrel) and the counter pressure on the projectile from atmospheric pressure are negligible.

Let us first examine a typical launcher setup, shown in figure 1-3. The chamber is filled with a propellant. This may be a reactive substance, such as gun powder, or a compressed gas. A diaphragm or shear disc may be used to separate the chamber and launch tube sections. These are usually designed to burst or shear when the desired pressure has been reached in the chamber.

Applying Newton's Second law to the projectile, the velocity of the projectile when it exits the launch tube is

$$
V=\sqrt{2 \bar{p} A_{1} L_{1} / M}
$$

where $\bar{p}$ is the spatial average propelling pressure and $A_{1}$ is the launch tube's crosssectional area. So increasing the $A_{1} L_{1} / M$ ratio will yield higher velocities. This 
implies changing launch tube length and projectile mass and size. However, there are practical limits as to how large this ratio can be made, and there may be size constraints on the launcher or specific projectile size and mass requirements. The only other method of increasing projectile velocity is to increase the average propelling pressure.

High average pressures are difficult to attain with conventional powder propellants. Burning the propellant initially increases the pressure until it reaches a peak. The pressure then decays down as the projectile accelerates and the burned propellant expands. Likewise for preburned propellant launchers (launchers in which the projectile moves only once all the propellant has reacted or where the propellant is a compressed gas), the pressure is initially at a peak value, then decays rapidly as the propellant expands. The result is an average pressure well below the peak pressure achieved. Increasing the peak pressure would increase the average pressure, but the strength of the launcher components (chamber, launch tube, projectile) limit the peak pressure.

As the projectile accelerates, the propellant will expand. This generates rarefactions waves (waves which lower pressure) which travel from the projectile to the breech (the wall at far end of the chamber). Once these rarefactions reflect off the breech, they will then travel toward the projectile. Once they catch up to the projectile, they will lower the pressure behind it. It is therefore advantageous to delay the time it takes for these rarefactions to reach the projectile by having a longer chamber. If the area of the chamber is greater than that of the launch tube, we say the launcher is "chambered," or has chambrage. Chambrage helps keep the pressure on the projectile higher since rarefactions propagating though the expanding propellant 
are partially reflected as compression waves. This is because the gas in the chamber is expanding into a smaller volume.

Other than the design of the launcher, the properties of the propellant are of great importance. One of these properties is the acoustic impedance, $c \rho$, where $c$ is the speed of sound and $\rho$ is the density of the propellant. The acoustic impedance is sometimes referred to as the gas's inertia, since it represents the ability of the gas to expand quickly. This term is directly related to the pressure drop $d p$ for a given velocity change $d u$. The pressure drop across an acoustic rarefaction wave is given by

$$
d p=c \rho d u
$$

So a lower acoustic impedance will result in a lower pressure drop for a given projectile velocity increase. Assuming the propellant is an ideal gas, the pressure variation behind the projectile can be written as a function of $c_{0} \gamma u$, and is approximately given by the equation

$$
\frac{p}{p_{0}}=e^{-\gamma u / c_{0}}
$$

where $c_{0}$ is the initial sound speed of the propellant, $p$ is the pressure behind the projectile, and $u$ is the velocity of the projectile [14]. This means that a lower $\gamma$ and a higher $c_{0}$ will lead to a smaller pressure drop, but changing the initial sound speed is more effective and practical. The sound speed for an ideal gas is given by

$$
c=\sqrt{\gamma R T}=\sqrt{\gamma \frac{\tilde{R}}{M W} T}
$$

where $\tilde{R}$ is the universal gas constant and $M W$ is the propellant's molecular weight. Therefore, a light gas, such as helium and hydrogen, at a high temperature is preferable as a propellant for achieving very high projectile velocities. 
Finally, as is explained in [14], the dimensionless parameter $G / M$, where $G$ is the mass of the driver gas and $M$ is the mass of the projectile, is an important factor in launcher performance. The higher this ratio is, the better the launcher performance.

This makes sense since a larger $G / M$ ratio means any or all of the following: a lighter projectile, a higher initial propellant pressure, a longer chamber, and a larger chamber. All of these were shown to result in increased launcher performance.

\subsubsection{The Linear Explosive Driver Used as a Hypervelocity Launcher Ideal Linear Explosive Launcher Operation}

When used as a hypervelocity launcher, the linear explosive driver discussed in section 1.1 is followed by a chamber and a launch tube, inside of which is a projectile. The precursor shock generated by the imploding pump tube travels down the tube, bringing the light driver gas to high pressure and temperature. It then reflects off the projectile, or the area change section depending if the launcher is chambered or not, which brings the driver gas to an even higher pressure. This high pressure and temperature light gas serves as a propellant for the projectile. See figure 1-4 for a schematic of the launcher operation.

We can once again use normal shock relations to calculate the pressure and speed of sound of the driver gas after the reflection of the incident shock. These relations will be the same as those used to find the shocked state, only they must be applied to the shocked state to find the reflected state. If we take the example of part 1.1.1 and use nitromethane as the explosive and helium at 20 atm and $300 \mathrm{~K}$ as the driver gas, the reflected state of the driver gas would be at about $9000 \mathrm{~atm}$ and $14000 \mathrm{~K}$.

The shock wave will continue to reverberate between the projectile and the driver's virtual piston, and continue to increase the pressure on the projectile. 


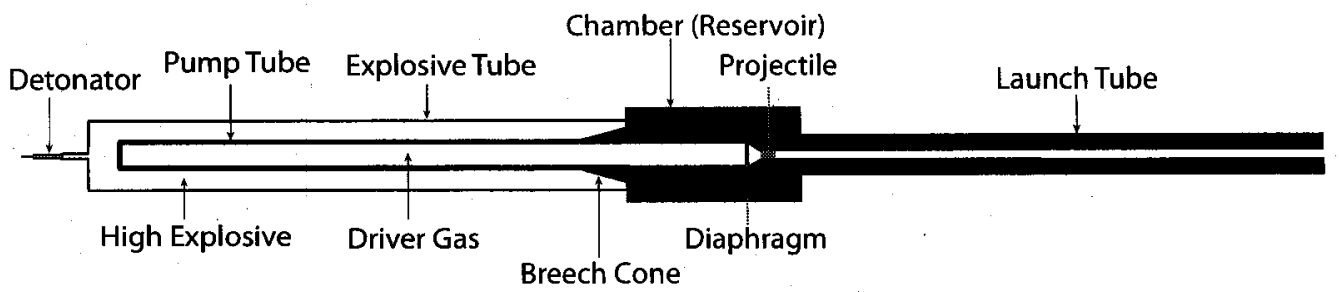

(a) Launcher before detonation

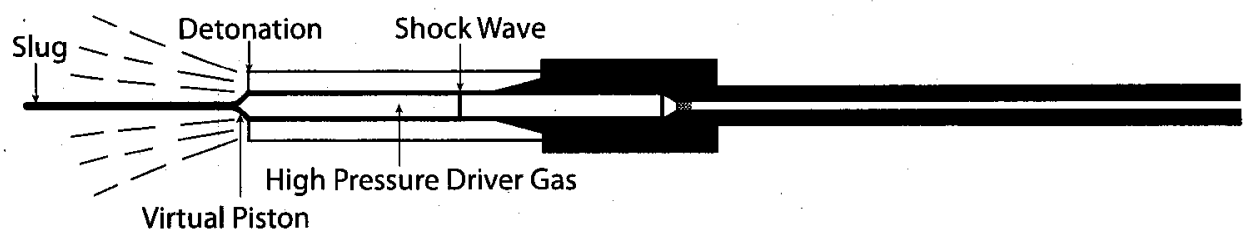

(b) Launcher during operation

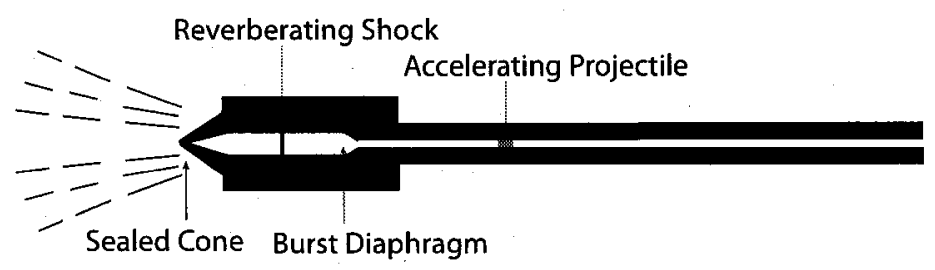

(c) Launcher firing projectile

Figure 1-4: Operation of the implosion-driven launcher

\section{Actual Linear Explosive Launcher Operation}

As was done for the linear explosive driver, Moore [10], Watson [17], and Crosby and Gill [4] performed experiments to evaluate the performance of the implosiondriven launcher.

As mentioned above, it is possible to generate very high driver gas pressures, high enough that the $G / M$ ratio of the launcher is effectively infinite. This yields very high projectile velocity estimates. However, such high pressures lead to the expansion and possible rupture of the launcher's chamber and launch tube, and could damage or destroy the projectile upon launch. The peak pressure is therefore limited by the strength of the launcher and projectile materials. It is also discussed in section 1.1.2 that the driver cannot generate arbitrarily long columns of shocked 
gas, which limits $G$. This means that the infinite $G / M$ assumption does not hold in experiments.

Ideally, once the driver's virtual piston reaches the chamber, it remains there and forms the breech of the chamber. Since driver's pump tube is usually a thin-walled tube, the virtual piston is not heavy enough to contain the high pressures inside the chamber. To this end, a breech cone was added at the junction of the pump tube with the chamber, as shown in figure 1-4. Once the detonation passes over the cone, it collapses it, ideally sealing the propellant gas in the breech. However, there is a limit as to how high the chamber pressure can be before the cone starts to fail. Watson [17] investigated the case where the reflected shock destroys the piston when they collide, which represents the absence or failure of the breech cone. The case where the reservoir remains intact after shock reflection is considered in Seigel [14] and scales with the $G / M$ ratio. Watson reports that, for the case where the piston vanishes, "several computer solutions have been nondimensionalized and shown to correlate, not with the $G / M$ ratio, but rather with a dimensionless reservoir length parameter $L=x / x_{0}$ where $x_{0}=$ reservoir length and $x=$ launch tube length" [17]. This makes sense, since the rarefactions generated by the loss of driver gas from the back of the chamber will take more time to reach the projectile if the chamber is longer.

Watson also noticed erosion of the walls of the reservoir and launch tube caused by the high temperatures reached by the driver gas. Erosion of the launcher walls mixes heavy metal particles to the driver gas, which increases the molecular weight. This increases the acoustic impedance of the driver gas and entails a loss in performance. The temperature reached by the driver gas depends on its initial temperature 
and the strength of the shock. Decreasing the detonation velocity of the explosive would lead to lower temperatures, but also lower pressures, in the driver gas.

As mentioned above, the shock wave will keep reverberating between the projectile and the piston. The shock reflections will produce a series of peaks on the pressure history of the projectile. In order to minimize the peak pressure experienced by the projectile, these pressure spikes should be of the same magnitude. Crosby and Gill [4] discovered empirically that this happens when the $G / M$ ratio is unity. The magnitude of the pressure spikes are then determined by the piston velocity and the initial driver gas pressure.

Other experiments done by Watson [17] showed that placing the projectile right up against the area change section would lead to damage to the projectile. A two dimensional Eulerian-Lagrangian computer program showed that the pressure on the projectile face was not uniform in this case, with a large pressure concentration in the center. This was confirmed by high speed photography showing a hole bored through the center of the projectile launched in experiments where the projectile was located at the area change section. Moving the projectile three diameters away from the area change section resulted in uniform pressure on the projectile in the calculations and an intact projectile in experiments. Damage to the front of projectiles was also observed and "attributed to violent muzzle release and flight of the projectile into air at 1 atm" [17]. Firing the projectile into a light gas such as helium should lead to a gentler firing.

A one-dimensional hydrodynamic computer program capable of simulating area changes was used in [10] to approximate the gas-dynamics of the driver gas inside the launcher and predict the acceleration history of the projectile. Experiments were done where the projectile, the shock in air ahead of it, and the helium driver gas 
behind it were observed with a high-speed streaking camera through viewing ports made in the launch tube of the launcher. Recorded experimental velocities were lower than predicted due to non-ideal effects. The trajectory of the piston was then artificially modified in the simulation so that the calculated shock trajectory closely matched the measured experimental trajectory. A fictitious piston traveling slower than detonation velocity which stopped and then reversed direction was used in the simulation rather than a piston traveling at the detonation speed. 'This was done to account for the non-ideal effects in the pump tube operation. It was assumed that if the calculated trajectory matched that observed in experiments, so too must the gasdynamic conditions of the driver gas.

The main reason the code used in [10] over-estimated projectile velocities when using a piston representative of the actual detonation trajectory was believed to be that the reservoir expands in experiments. The code was modified by Watson [17] to account for reservoir expansion by sectioning the wall into discrete cross-sections and running a one-dimensional Lagrangian calculation in cylindrical geometry for each cross section, then having the wall motion affect the pressure inside the reservoir. An experiment was done and the velocity and exit time of the projectile agreed extremely well with the results of the corresponding simulation using this new version of the code. This code also revealed that significant increase in performance could be achieved by using a layer of explosives around the reservoir in order to prevent expansion. This is called an auxiliary pump cycle. See figure 1-5 for a schematic of the auxiliary pump. The explosives in the auxiliary pump are initiated when the incident shock enters the reservoir, applying very high pressure on the outside of the chamber to counter expansion. Calculated and measured experimental velocities using the auxiliary pump agreed very well and were close to the ideal case. 


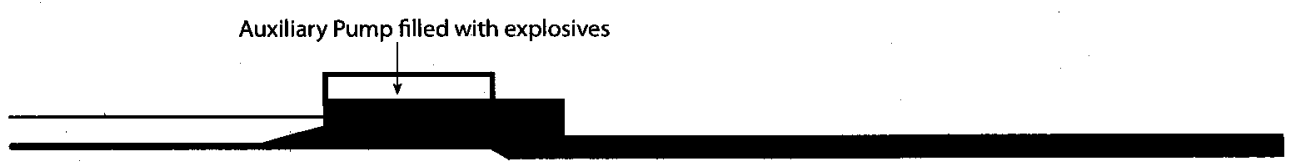

Figure 1--5: Auxiliary pump schematic

\subsection{Present Study}

Although many different studies and experiments were performed on the linear explosive driver and launcher, no rigorous engineering treatment has been made. The details on how to go about constructing these devices are also unclear in all the mentioned literature; work done and techniques used are presented, but design criteria and general trends are not always stated. Few systematic studies of varying a single parameter (e.g. fill pressure) were reported. The present study was aimed at building on this prior work in hopes of establishing these criteria and trends, and also of improving the capabilities of these devices. To this end, numerical codes were used to estimate performances, and systematic experiments were carried out on both the driver section and the single-stage launcher to ascertain the effects of various parameters. 


\section{CHAPTER 2 \\ Expanding Tube Model}

The purpose of the pump tube is to generate a reservoir of hot, high-pressure driver gas which can be used as the propellant for the launch cycle of a hypervelocity launcher. This is done by surrounding the tube with explosives which linearly implode the tube, driving a strong precursor shock through the driver gas. The post-shock pressures after the precursor shock are such that the stresses generated in the pump tube typically surpass the yield strength of the material, leading to pump tube expansion. Expansion of the tube walls generates rarefactions which weaken the precursor shock and hinder the performance of the driver. The shock pressures generated may even exceed the bursting pressure of the tube. If the tube bursts before the detonation has had a chance to implode it, loss of driver gas results in reduced launcher performance. It is therefore important to understand the dynamics of pump tube expansion. In so doing, it will be possible to gauge the effects of the various parameters influencing the problem. These include the initial driver gas fill pressure, the pump tube radius, the thickness of the pump tube, the thickness of the explosive layer, and the material properties of the tube and the explosive. It would also help quantify the effect of adding a tamper surrounding the explosive.

Because of the dynamic nature of the device, the tube may not reach a static equilibrium state. The tube may be expanding until it is collapsed by the detonation wave; what is important is to minimize this expansion and to prevent bursting. The role of the tamper in this regard is a dynamic one: an adequate pressure has to build up between it and the pump tube before the tamper can have a noticeable effect. 


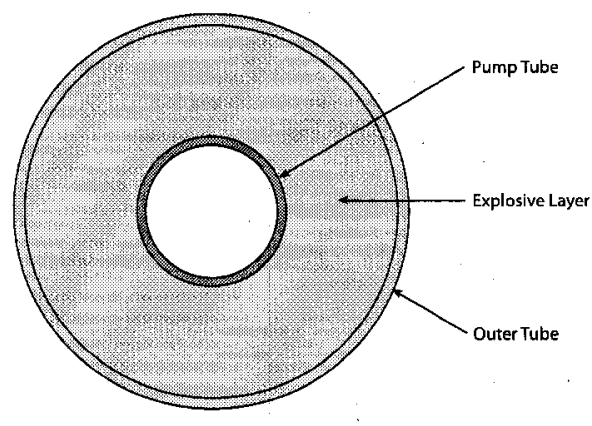

(a) Untamped

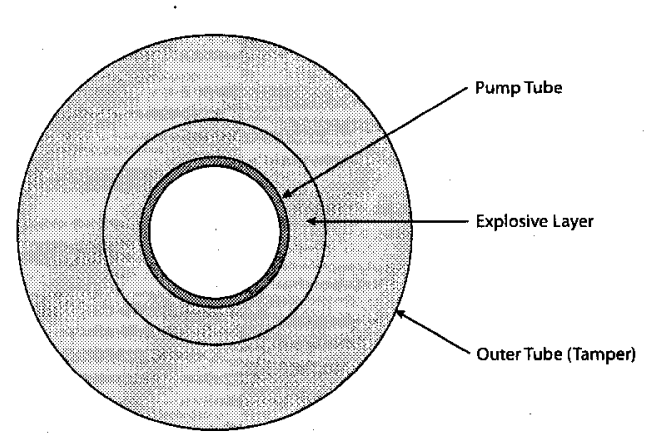

(b) Tamped

Figure 2-1: Typical driver cross sections

The static equations are therefore not enough to model these effects, and we need a completely dynamic model.

\subsection{Derivation of the Model}

\subsubsection{Pump Tube Motion}

Consider a two-dimensional cross sectional "slice" of the driver, located some distance $x_{0}$ down the tube. See figure 2-1 for typical cross sections of the driver tube, both tamped and untamped. Let us start by examining a cross section of the pump tube. The tube will be subjected to an instantaneous change in internal pressure once the shock wave passes through $x_{0}$. We are interested in the behaviour of the cross section of the tube as time progresses after it has been subjected to post-shock pressure. The problem is axisymmetric, which means that the tube will expand uniformly and remain circular. Let us assume that the inclination of the tube wall with respect to the axis of symmetry is negligible. This is analogous to a cylindrical piston which is driven outward via a constant internal pressure. We can then examine a one-dimensional segment of the cross section. Let us take our tube segment and apply Newton's Second law. See figure 2-2 for a sketch of the tube segment and forces acting on it. 


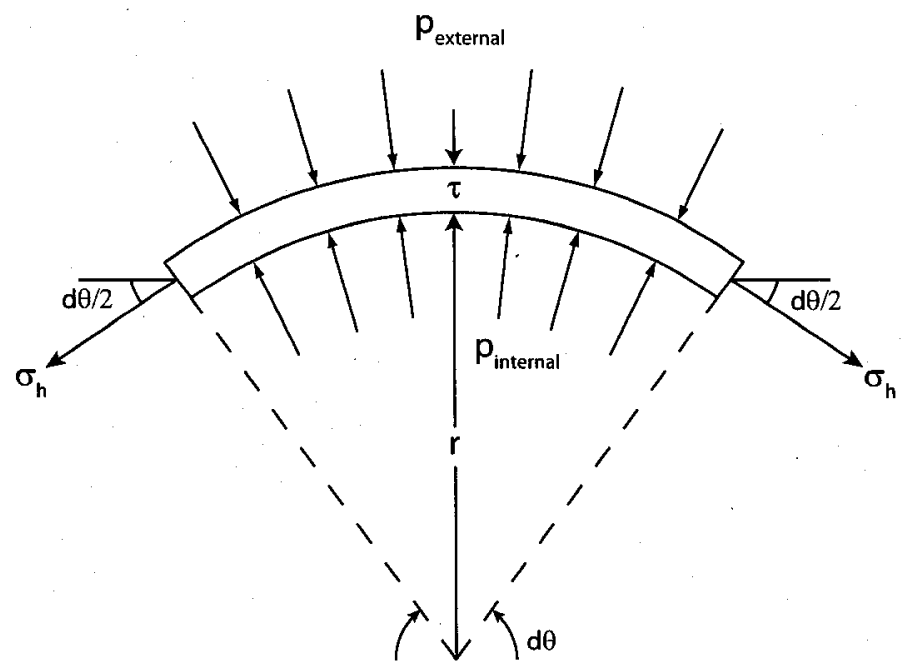

Figure 2-2: Force balance on pump tube segment

We are looking at an elementary segment, so the angle $d \theta$ is small. The pump tube has radius $r$ and thickness $\tau$. Since the pump tube is typically a thin-walled tube, we can treat it as a thin-shelled cylinder. We therefore only consider the hoop stress $\left(\sigma_{h}\right)$ and assume it is constant throughout the thickness. The tube is experiencing both internal pressure $p_{\text {internal }}$, from the shocked driver gas contained within, and external pressure $p_{\text {external }}$, from the liquid explosive surrounding it which is being compressed by the expanding tube. Applying Newton's Second law in the radial direction and using the small angle approximation $\left(\sin \left(\frac{d \theta}{2}\right) \approx \frac{d \theta}{2}\right)$, we obtain the following differential equation:

$$
\ddot{r}=\frac{\left(p_{\text {internal }}-p_{\text {external }}\right)}{\tau \rho_{\text {tube }}}-\frac{\sigma_{h}}{r \rho_{\text {tube }}}
$$

where $\rho_{\text {tube }}$ is the density of the tube material. The thickness of the tube will decrease as the tube expands. If we assume the tube material is incompressible, we obtain

$$
\tau=\frac{r_{0} \tau_{0}}{r}
$$


where $r_{0}$ is the initial pump tube radius and $\tau_{0}$ the initial pump tube thickness.

The pressure in the explosive layer $p_{\text {external }}$ will be influenced by the motion of both the pump tube and the outer tube as well as the distance between both tubes. So $p_{\text {external }}$ will be a function of $r_{p t}, \dot{r}_{p t}, r_{o t}$, and $\dot{r}_{o t}$, where the subscripts $p t$ and $o t$ refer to the pump tube and the outer tube respectively. This will be explained in section 2.1.4. The stress $\sigma_{h}$ will depend on both the strain and the strain rate in the pump tube. So $\sigma_{h}$ will be a function of $r$ and $\dot{r}$, as discussed below.

Substituting equation 2.2 into equation 2.1 and noting the functional dependencies of $p_{\text {external }}$ and $\sigma_{h}$ we obtain the following:

$$
\ddot{r}_{p t}=\left[\frac{\left(p_{\text {internal }}-p_{\text {external }}\left(r_{p t}, \dot{r}_{p t}, r_{o t}, \dot{r}_{o t}\right)\right) r}{r_{0} \tau_{0} \rho}-\frac{\sigma_{h}(r, \dot{r})}{r \rho}\right]_{p t}
$$

As will be shown in section $2.1 .3, p_{\text {internal }}$ will be a constant. We can see here that the motions of the pump tube and the outer tube are coupled. As will be shown in section 2.1.2, we can derive a similar ordinary differential equation for the outer tube motion. This means the entire pump tube radial motion can be expressed as a system of ordinary differential equations.

\section{Hoop Stress in the Pump Tube}

We typically expect very high strain rates in the pump tube since we are instantaneously loading it with a very high pressure. Therefore, the conventional static values for stress and strain will not apply to our situation, and we must obtain a value for the hoop stress that will take into account this high strain rate (thus making stress a function of $r$ and $\dot{r}$ ). Johnson and Cook developed a constitutive model for metals subjected to large strains, high strain rates and high temperatures [5]. This model is widely used in similar problems, such as collapsing liners in shaped charge and explosively formed penetrator devices. For example, see [3]. 
The Johnson-Cook strength model defines the von Mises stress $\sigma$ as the following:

$$
\sigma=\left[A+B \varepsilon^{\eta}\right]\left[1+C \ln \dot{\varepsilon}^{*}\right]\left[1-T^{* m}\right]
$$

where $\varepsilon$ is the plastic strain, $A$ is the yield stress, $B$ and $\eta$ are constants which account for strain hardening, the terms in the second bracket account for the effect of strain rate, and the terms in the last bracket account for the effect of temperature. $\dot{\varepsilon}^{*}$ is the dimensionless plastic strain rate and is given by $\dot{\varepsilon}^{*}=\dot{\varepsilon} / \dot{\varepsilon}_{0}$, where $\dot{\varepsilon}_{0}=1.0 \mathrm{~s}^{-1}$. $T^{*}$ is the homologous temperature and is given by $T^{*}=\frac{T-T_{\text {room }}}{T_{\text {melt }}-T_{\text {room }}}$, where $T_{\text {room }}$ is the initial temperature of the material and $T_{\text {melt }}$ is the melting temperature of the material. All the constants used here - $(A, B, \eta, C$, and $m)$ - are determined from tension and torsion tests at different strain rates and temperatures. Note that these values are not readily available for any material, but the accepted values for several materials are given in [5]. The pump tube used in experiments was made of stainless steel 304, but since values for this material are not available, it was approximated as steel 1006.

Note that the Johnson-Cook model considers plastic values of stress only. It assumes that the elastic region is negligible, which is reasonable for high strain rates and large plastic deformations. The linear section of the stress-strain curve is then suppressed and we have a stress equal to the yield stress of the material starting from zero strain. The engineering strain is defined as the stretch of the material over its initial length:

$$
\varepsilon=\frac{\ell-\ell_{0}}{\ell_{0}}
$$


In our case, the length of the material is equal to the circumference of the tube: $\ell=2 \pi r$. The expression for strain then becomes:

$$
\varepsilon=\frac{2 \pi r-2 \pi r_{0}}{2 \pi r_{0}}=\frac{r-r_{0}}{r_{0}}=1-\frac{r}{r_{0}}
$$

Although the tube is initially at room temperature, it will heat up due to plastic work of deformation; we therefore cannot neglect temperature effects. The temperature change can be expressed as:

$$
\Delta T=\frac{\beta}{\rho C_{p}} W
$$

where $\beta$ is the work rate to heat rate conversion factor, and is empirically determined to be about equal to 0.9 for most cases, $\rho$ is the density of the material, $C_{p}$ is the heat capacity of the material, and $W$ is the plastic work of deformation. We can obtain $W$ by integrating the stress-strain curve:

$$
W=\int_{0}^{\varepsilon_{f}} \sigma d \varepsilon
$$

where $\varepsilon_{f}$ is the final strain reached. Since this model neglects the elastic region and considers only plastic behaviour, it is important to check that the pressure inside the tube is high enough to bring it into the plastic regime. However, a quick check shows that, for most cases, we quickly enter the plastic region and the effect of the elastic region on tube motion is negligible. It is also important to note that, since we are in the plastic regime, the stress in the tube will always oppose the motion. If the tube motion reverses direction, so too will the stress. This is also important when integrating the stress-strain curve to obtain $W$ : it is not enough to integrate from zero strain until the current strain; any deformation of the tube, expansion and compression alike, will generate heat, so the current value of temperature is path 
dependent. We can now come up with an appropriate value for the hoop stress in the pump tube in equation 2.3 .

\subsubsection{Outer Tube Motion}

The differential equation governing the outer tube motion will be very similar to equation 2.3, except it will only be subjected to the internal pressure from the liquid explosive it contains:

$$
\ddot{r}_{o t}=\left[\frac{p_{\text {internal }}\left(r_{p t}, \dot{r}_{p t}, r_{o t}, \dot{r}_{o t}\right) r}{r_{0} \tau_{0} \rho}-\frac{\sigma_{h}(r)}{r \rho}\right]_{o t}
$$

where the subscript ot designates the outer tube.

If we are dealing with the untamped case, typically a thin plastic tube is used to contain the explosive, so we may neglect its strength $\left(\sigma_{h}=0\right)$. Complications arise when dealing with the tamped case. A tamper is a thick-walled tube by nature, and so our thin shell approximation is no longer valid. Equations for inner wall motion, stress, and strain are readily available for quasi-static loadings of thick-walled tubes in the elastic regime. However, it is surprisingly difficult to obtain dynamic equations for high strain rates and large plastic deformations for such tubes. Typically, finite element methods are used for simulating these cases, which is not within the scope of this simple analytic model. We can try using the thin-wall approximation in order to obtain an order of magnitude estimate of the dynamics of a thick-walled tube. However, we must keep in mind that the stresses in the tube and its inertia will not be accurately reproduced. In this case, it would be safe to design the tamper such that it will contain the maximum pressure it will be exposed to during the expansion phase: the shock pressure inside the pump tube. The mean diameter formula is commonly used to estimate bursting pressures of thick-walled tubes:

$$
p_{\text {burst }}=2 \sigma_{u} \frac{a-b}{a+b}
$$


where $a$ is the outer radius, $b$ is the inner radius, and $\sigma_{u}$ is the ultimate stress of the tube material.

We expect that, if the outer tube is a thick-walled tamper, it will respond much more slowly than the pump tube. Since we are effectively designing the tamper to avoid its motion altogether, the strain rates in the tamper will be much lower than in the pump tube, and in most cases the tamper will remain in the elastic regime. We may then model its stress by assuming a perfectly elastic region, followed by a perfectly plastic region once we reach yield stress. This is called a perfectly elasticplastic model, and the stress is a function of radius only in this case.

\subsubsection{Driver Gas Pressure}

The internal pressure for the pump tube is the pressure of the driver gas it contains. The initial condition used here is the shocked state of the driver gas, which can be easily calculated using normal shock relations given the initial fill pressure of the driver gas and the detonation velocity of the explosive.

The flow behind the shock is subsonic relative to the shock, which means that changes in pressure and velocity can be communicated through the flow and to the shock. Thus, the pressure experienced by the tube due to the post-shock gas tends to be uniform and, for a fixed shock strength, independent of expansion of the pump tube. This argument is reflected by findings made by Watson [17]. As mentioned in section1.1.2, Watson found that the pressure inside the pump tube remains relatively constant, the effect the tube expansion being compensated by shortening of the column of shocked gas [17]. However, the fact that the tube is expanding will be communicated to the shock, which will slow it down. A slower shock velocity means a lower pressure ratio across the shock, and this will in turn bring about a decrease in pressure of the shocked driver gas. 
For this simplified model, the shocked driver gas pressure will be computed using normal shock relations assuming ideal shock speed. This pressure will be held constant during the calculation.

\subsubsection{Explosive Layer Pressure}

The pressure in the explosive layer is not constant and will fluctuate depending on the movement of its boundaries. When the pump tube expands into the explosive, it will send pressure waves through it. These pressure waves are fairly weak since the fluid speed is small compared to the sound speed. The explosive used in the present study is nitromethane, which is a liquid. Given these conditions, the change in density and sound speed of the explosive will be negligible for the pressures it will be subjected to. Therefore, the pressure in the explosive layer can be modeled using acoustic waves. The problem of solving for pressure then consists of keeping track of all the pressure waves sent back and forth into the explosive layer. The use of acoustics simplifies the problem since they propagate at the speed of sound in the material, which is assumed constant, and therefore they are represented as straight lines in an $x-t$ diagram. Computationally this makes them easier to keep track of, as opposed to the more general method of characteristics. Their effects also add up linearly, which makes it easy to calculate the pressure at any point, provided that we have kept track of all the relevant acoustic waves up to that point.

Since we are looking at a one-dimensional slice of the tube, the expanding tube wall acts on the explosive very much like a piston would on a fluid in a pipe. Since the fluid next to the piston has to be traveling at the same velocity as the piston, a change in piston velocity $\Delta u$ is accompanied by a change in fluid velocity of equal magnitude. This generates a wave across which there is a change in fluid velocity $\Delta u$ which propagates through the fluid at the speed of sound $c_{0}$ of the fluid. The 
pressure change across this wave is given by

$$
p_{1}-p_{0}=\rho_{0}\left(u_{1}-u_{0}\right)\left(U-u_{0}\right)
$$

where $\rho_{0}$ is the fluid density, $u$ is the fluid velocity, $U$ is the wave speed, and the subscripts 0 and 1 refer to the states before and after the wave, respectively. This equation can easily be derived from the conservation of mass and momentum equations. In our case, the wave speed is the sound speed in the fluid, and the fluid speed is negligible when compared to the sound speed $\left(U-u_{0} \approx U\right)$. From equation 2.7, the change in pressure $\Delta p$ across an acoustic wave then becomes

$$
\Delta p=\rho_{0} c_{0} \Delta u
$$

It is easy to see that a movement of the pump tube into the fluid will raise the pressure, and a movement out of the fluid will lower the pressure. This gives the pressure in the explosive layer a dependence on $\dot{r}$.

The fact that the pump tube is expanding will be communicated through the fluid as acoustic waves. If the explosive layer is infinitely thick, these waves propagate outward forever, and the pressure on the tube surface will simply be the sum of each $\Delta p$ across all the acoustic waves generated. See figure 2-3 for a sketch of this case.

If the explosive layer cannot be considered infinitely thick (as is usually the case), the generated acoustics will reflect off the outer boundary of the explosive layer. For now, let us assume this outer boundary is rigid, as shown in figure 2-4. We have traced the trajectory of a single acoustic wave in this figure. After reflection off the outer boundary, the acoustics will then travel back and reflect off the pump tube, and so reverberate back and forth between both boundaries. We can think of these waves as carriers of information. Once the pump tube starts expanding, this 


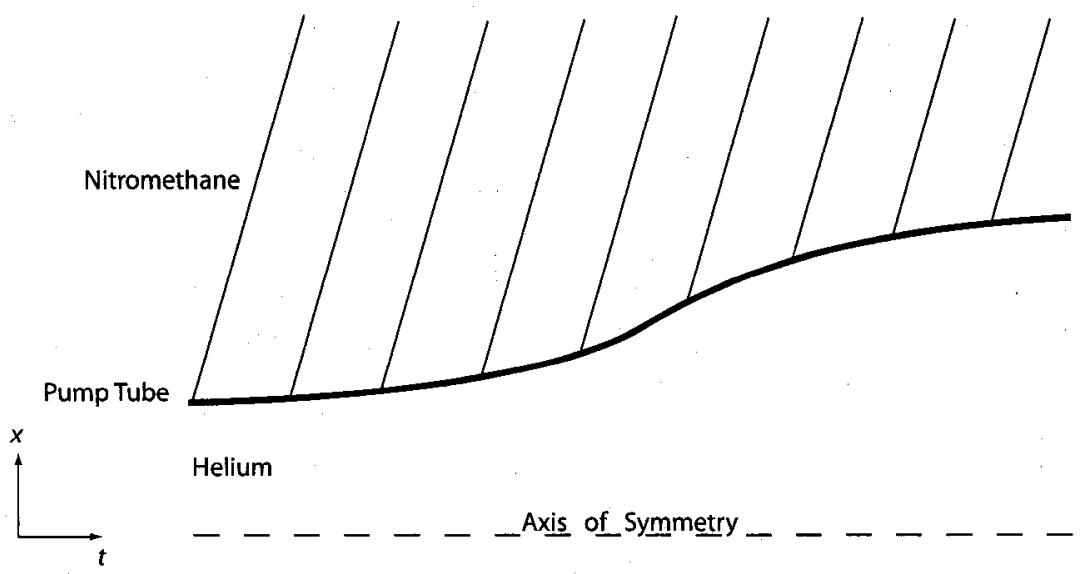

Figure 2-3: Position-time graph showing expanding pump tube and generated acoustics for an infinite explosive layer

information is communicated through the fluid in the form of acoustic waves. The outer boundary will only be aware of the movement of the inner boundary once these waves reach it. Then the waves will reflect back and communicate the presence of the outer boundary to the rest of the fluid. The effect of this information going back and forth is to adjust the speed, and therefore pressure, of the fluid in response to the disturbances it is experiencing.

Every time we cross an acoustic wave, we must increase the pressure of the fluid by an amount equal to the $\Delta p$ associated with that wave. Referring to figure $2-4$, region 1 is at a pressure $p_{0}+\Delta p$, region 2 is at a pressure $p_{0}+2 \Delta p$, and region 3 is at a pressure $p_{0}+3 \Delta p$. Note that the pump tube never actually experiences region 2 ; it goes directly from region 1 to region 3 . Likewise, the outer boundary never sees region 1 ; it goes directly from region 0 to region 2 . Therefore, as far as the 


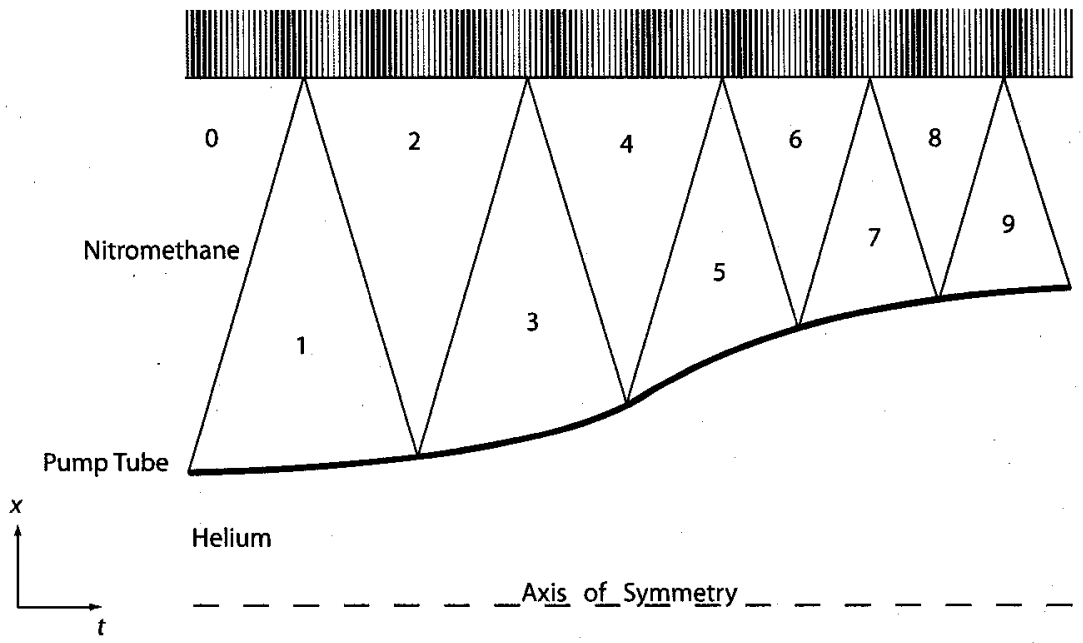

Figure 2--4: Position-time graph showing expanding pump tube and acoustic reverberations. Outer boundary is a rigid tamper.

boundaries are concerned, when a wave reflects off of them, the pressure increases by an amount equal to twice the $\Delta p$ associated with that wave, so for the pump tube: $p_{3}=p_{1}+2 \Delta p, p_{5}=p_{3}+2 \Delta p$, and so on.

The case described above is for a single acoustic wave. More generally, there will be a continuum of acoustic waves sent out along the entire surface of the pump tube. For this simplified model, there will be one acoustic wave sent out per timestep, and the time-step size will be of the order of 1 to 10 ns. Displaying all the acoustics present in figure 2-4 would result in an overly cluttered graph. Instead, let us examine a zoomed in portion of the graph and study what happens during one time-step, as shown in figure 2-5. Shown here are the acoustic waves sent out at times $t$ and $t-\Delta t$ and we assume that there are two acoustics which reflect off the pump tube in between those times. We now wish to know what the pressure is at 


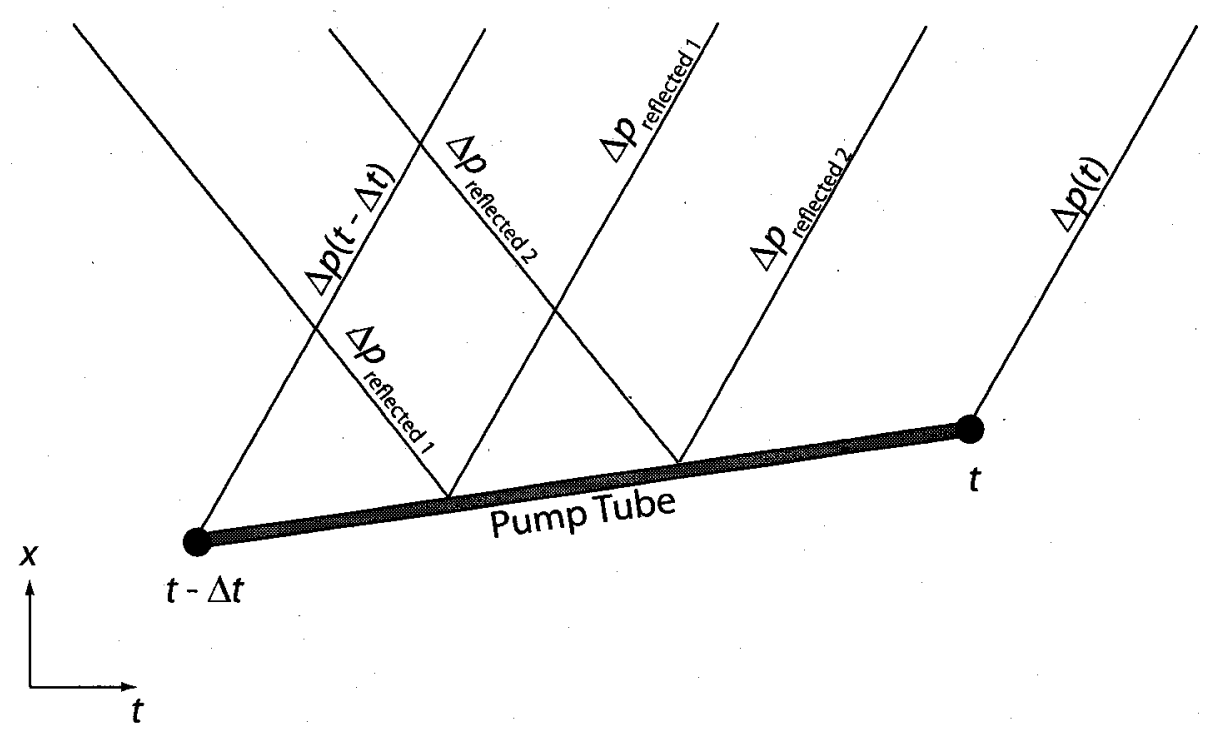

Figure 2-5: Zoom of the position-time graph of the expanding tube showing one time-step

time $t$. To do this we must know the conditions at time $t-\Delta t$ and everything that has happened since then. At time $t-\Delta t$, the pressure is $p(t-\Delta t)$, the tube radius is $r(t-\Delta t)$ and the wall speed is $\dot{r}(t-\Delta t)$. Based on the difference in internal and external pressures at time $t-\Delta t$, we can calculate the new radius and wall speed at time $t, r(t)$ and $\dot{r}(t)$, by solving equation 2.3. The change in wall speed will generate an acoustic at time $t$ with a $\Delta p$ given by

$$
\Delta p(t)=\rho_{0} c_{0} \Delta u(t)=\rho_{0} c_{0}(\dot{r}(t)-\dot{r}(t-\Delta t))
$$

We also need to account for the increase in pressure due to the reflected waves. We saw above that when a wave reflects off of the pump tube, the pressure increases by an amount equal to twice the $\Delta p$ associated with that wave. We then need to add $2\left(\Delta p_{\text {reflected }_{1}}+\Delta p_{\text {reflected }_{2}}\right)$ to the pressure at time $t$. The pressure at time $t$ is then given by:

$$
p(t)=p(t-\Delta t)+\Delta p(t)+2\left(\Delta p_{\text {reflected }_{1}}+\Delta p_{\text {reflected }_{2}}\right)
$$


Since the effects of the acoustic waves add up linearly, we do not necessarily need to keep track of every individual wave generated since the beginning of the calculation. Once these waves interact with a boundary, we can superimpose their effect to the next wave sent out from that boundary. In other words, we assume the reflected waves in our example all interact with the pump tube at time $t$. The acoustic sent out from the pump tube at time $t$ will therefore include the effects of all the reflected waves as well as the change in wall speed. We therefore diminish the number of waves we have to follow by stacking them together, and thus simplify the calculation. The error incorporated by using this technique is negligible due to the very small step size and the approximate and discrete nature of the model. In the current example, this means that the wave sent out from the tube at time $t$ has a total $\Delta p$ given by:

$$
[\Delta p(t)]_{\text {tot }}=\Delta p(t)+\Delta p_{\text {reflected }_{1}}+\Delta p_{\text {reflected }_{2}}
$$

Note here that we have not added twice the effect of the reflected waves. This is because waves are reflected as waves having the same strength. So a wave having a certain $\Delta p$ will reflect off a boundary as a wave with that same $\Delta p$ (as shown in figure $2-5$ ), even though its effect on the pressure of the fluid at the boundary will be to increase it by $2 \Delta p$.

More generally, there will be an arbitrary amount of reflected waves during one time-step, so the equations given above become:

$$
p(t)=p(t-\Delta t)+\Delta p(t)+2 \sum \Delta p_{\text {reflected }}
$$

and

$$
[\Delta p(t)]_{t o t}=\Delta p(t)+\sum \Delta p_{\text {reflected }}
$$


where $\Delta p(t)$ comes from the pressure wave generated at the tube at time $t$, and the term $\sum \Delta p_{\text {reflected }}$ is the cumulative pressure contribution from all the waves which were reflected off the tube during the time-step $\Delta t$. In this example, we have considered only the pump tube, but the same arguments and equations hold when considering the outer tube.

The $\Delta p_{\text {reflected }}$ terms were generated at an earlier point in time, so the pressure at a certain point in time is a function of the pressure at an earlier point in time. The differential equations 2.3 and 2.6 then become what are known as delay differential equations, or DDEs. The delay caused by an acoustic wave having to travel back and forth between both tubes is called the communication time $\left(t_{c}\right)$. The communication time is equal to the time it takes for an acoustic wave to travel between both tubes. This time is not constant since the distance between both tubes is not constant; it is a function of the radii of the pump tube and the outer tube, which are functions of time. A wave reaching the outer tube at some time $t$ was generated at the pump tube at some time $t-t_{c}$ earlier, so we must know what the radius of the pump tube was at time $t-t_{c}$. In this case, $t_{c}$ is given by:

$$
t_{c}=\frac{r_{o t}(t)-r_{p t}\left(t-t_{c}\right)}{c}
$$

A wave reaching the pump tube at some time $t$ was generated at the outer tube at some time $t-t_{c}$ earlier, so when considering a wave traveling from the outer tube to the pump tube, $t_{c}$ is given by:

$$
t_{c}=\frac{r_{o t}\left(t-t_{c}\right)-r_{p t}(t)}{c}
$$


This gives the pressure in the explosive layer a dependence on $r$ and $\dot{r}$ of both the pump tube and the outer tube. The functions for $t_{c}$ are implicit. In the numerical solver, we will have to iterate to find $t_{c}$.

If we now allow the outer tube to move in reaction to the pressure it is exposed to, its motion will generate acoustics just as the pump tube motion does. If this tube has a low strength and low inertia, it will quickly expand due to the pressure applied to it. This will send waves which lower pressure, or rarefaction waves, into the fluid. Once these waves reach the pump tube, they will lower the pressure on its surface and allow it to expand faster. In order to help prevent pump tube expansion, it is therefore best to limit the expansion of the outer tube, the ideal case being a rigid outer tube, as in figure 2-4. This is where the concept of the tamper demonstrates its utility. If we use a heavy-walled tube made of a strong material, such as steel, to contain the explosive, it will better contain the pressure within it, thus delaying pump tube expansion. Also, the effect of the tamper will be "felt" by the pump tube more quickly if the communication time is small, so a thin layer of explosive is best when using a tamper. We can now obtain a reasonable value for the pressure in the explosive layer, both on the pump tube and the outer tube,

\subsubsection{Summary}

Let us now summarize what we have done. We have found a differential equation governing the pump tube motion in equation 2.3, which accounts for both the inertia and the strength of the tube. We can apply this equation to the outer tube as well and obtain equation 2.6, but it is worth reiterating that it uses the thinwall approximation and does not accurately reproduce the dynamics of thick-wall tampers. 
We can obtain the stress in the pump tube, $\sigma_{h_{p t}}$, from the Johnson Cook strength model described above, since the pump tube is subjected to high strain rates. If the purpose of the outer tube is solely to contain the explosive, we may neglect its strength $\left(\sigma_{h_{o t}}=0\right)$. If the outer tube is a thick-wall tamper, we may find its stress using a more conventional perfectly elastic-plastic model since it is slower to respond and will usually remain in the elastic regime.

The internal pressure in the pump tube, $p_{\text {internal }_{p}}$, due to the shock wave traveling in the driver gas is a constant and can be obtained through normal shock relations, given the piston velocity and the initial state of the gas inside the pump tube. The external pressure for the pump tube, $p_{\text {external }}$, and the internal pressure for the outer tube, $p_{\text {internal }_{o t}}$, due to compression of the explosive layer may be solved by the acoustic method described above. We can now integrate the system of differential equations numerically. The algorithm used to solve the system of differential equations is presented in Appendix A

\subsection{Model Results}

\subsubsection{Calculated Expansion Histories}

The differential equations governing the pump tube and outer tube motions were integrated using a fourth-order Runge-Kutta method. The stresses and pressures were obtained using a forward difference method within the main loop of the calculation. In all cases, convergence was verified by altering the time-step size until the results were found to converge.

The code outputs the pump tube radius and the outer tube inner radius as a function of time. It is then easy to alter various parameters, like pump tube diameter or initial fill pressure, and see their effects on the expansion of the pump tube. 
A design requirement is that the pump tube does not burst during the time it takes for the detonation to sweep over the tube after the shock has passed. Since the shock is traveling faster than the detonation, the longer the pump tube is, the farther ahead of the detonation the shock will get, and the longer it will have to contain the pressure without bursting. However, it is difficult to predict when the pump tube will burst under conditions of high strain rate and large plastic deformations. A safe criterion was deemed to be about $30 \%$ elongation: if the pump tube circumference expands over $30 \%$ of its initial value, it is considered that the tube fails.

Figure 2-6 shows expansion histories for a $6.35 \mathrm{~mm}$ outer diameter tube with a $0.9 \mathrm{~mm}$ wall thickness. The explosive layer thickness is $3.1 \mathrm{~mm}$. The tube is $1 \mathrm{~m}$ long, so in this case the tube has to hold pressure for about $45 \mu \mathrm{s}$. Figure 2-6(a) shows the results for an untamped driver, and figure 2-6(b) shows results for a tamped driver. The tamper has an inner radius of $0.96 \mathrm{~cm}$ and an outer diameter of $2.22 \mathrm{~cm}$. The bursting pressure for such a tamper is about $45 \mathrm{~atm}$. The tamper is assumed to behave according to the perfectly elastic-plastic model in the calculation. For the untamped case, the pump tube reaches $30 \%$ elongation before $45 \mu$ s for all pressures shown here. However, for the tamped case, the tamper is capable of dynamically containing the pump tube, which does not reach $30 \%$ elongation until beyond $45 \mu \mathrm{s}$ for all cases. This clearly shows that a tamper can make a substantial difference in preventing pump tube bursting. One thing to note though is that for $60 \mathrm{~atm}$, the tamper is not thick enough to contain the shock pressure in the driver gas. Since, as discussed above, the results obtained for the tamper motion are not exact, it should be made thicker if attempted experimentally. 


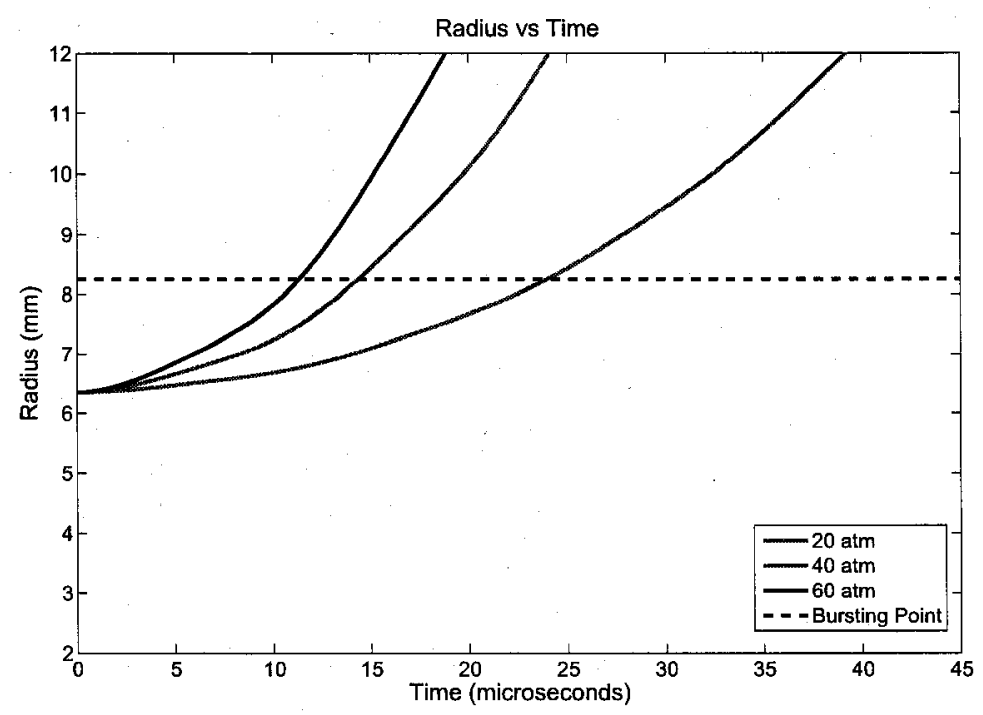

(a) Untamped driver

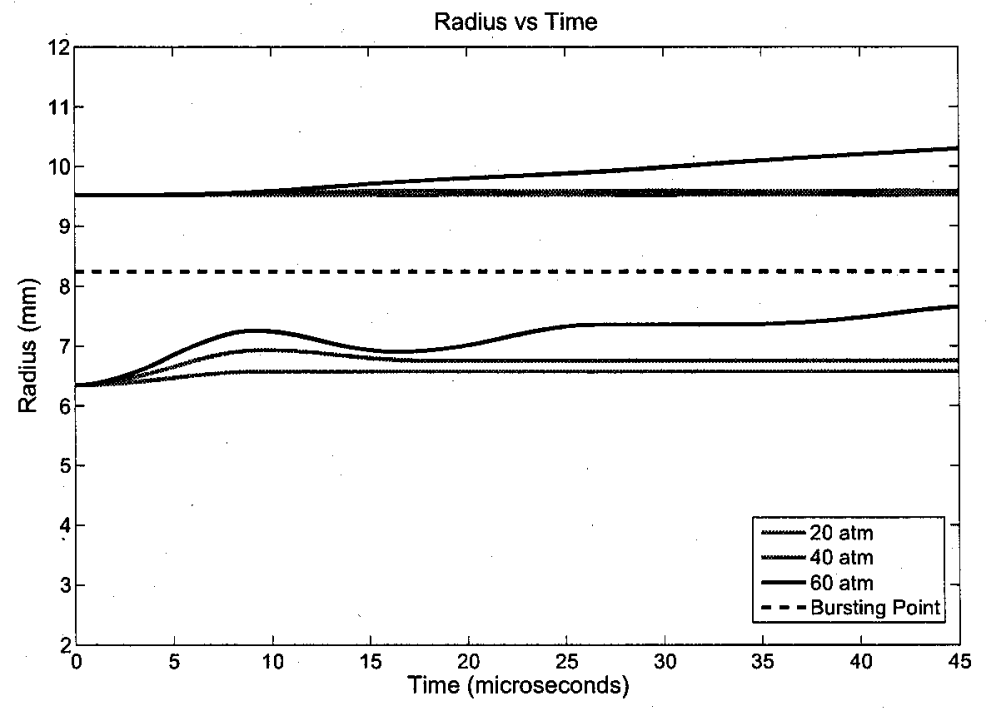

(b) Tamped driver

Figure 2-6: Expansion histories for various pressures 
Expansion histories such as these are not meant to predict exactly when the tube will burst or not, but they are intended to provide guidance when designing experiments, and offer insight into the dynamics and the general trends to be expected. By fixing some parameters and allowing others to vary, we can generate conceptual parametric "maps" and define regions of expected behaviour. See figure 2-7 for examples of such maps.

Figure 2-7(a) shows the effect of varying initial fill pressure for different pump tube radii. Here the pump tube thickness is $0.9 \mathrm{~mm}$, the explosive thickness is $3.175 \mathrm{~mm}$, the untamped case uses a PETG plastic tube having a thickness of $3.175 \mathrm{~mm}$, and the tamped case uses a steel tube having a thickness of $12.7 \mathrm{~mm}$. The tamper is assumed to behave according to the perfectly elastic-plastic model in the calculation. This plot is constructed for conditions at $1 \mathrm{~m}$ down the pump tube, measured from the detonation initiation end. We can see that for untamped tubes, a smaller radius is better able to contain pressure. Adding a tamper raises the maximum allowable initial fill pressure for any pump tube radius. However, the curve shows that tampers are less effective for smaller pump tube radii and more effective for larger pump tube radii, for a given explosive layer thickness. This indicates that the relative size of the pump tube and tamper is important. Since a smaller tube will bust a short time after expansion begins, the tamper needs to be relatively closer to the pump tube so that its effect is felt sooner.

Figure 2-7(b), shows the effect of varying initial fill pressure for different explosive layer thicknesses. Here the pump tube radius and thickness are $6.35 \mathrm{~mm}$ and $0.9 \mathrm{~mm}$, the untamped case uses a PETG plastic tube having a thickness of $3.175 \mathrm{~mm}$, and the tamped case uses a steel tube having a thickness of $12.7 \mathrm{~mm}$. We are again examining a cross-section $1 \mathrm{~m}$ down the pump tube. We can see that 


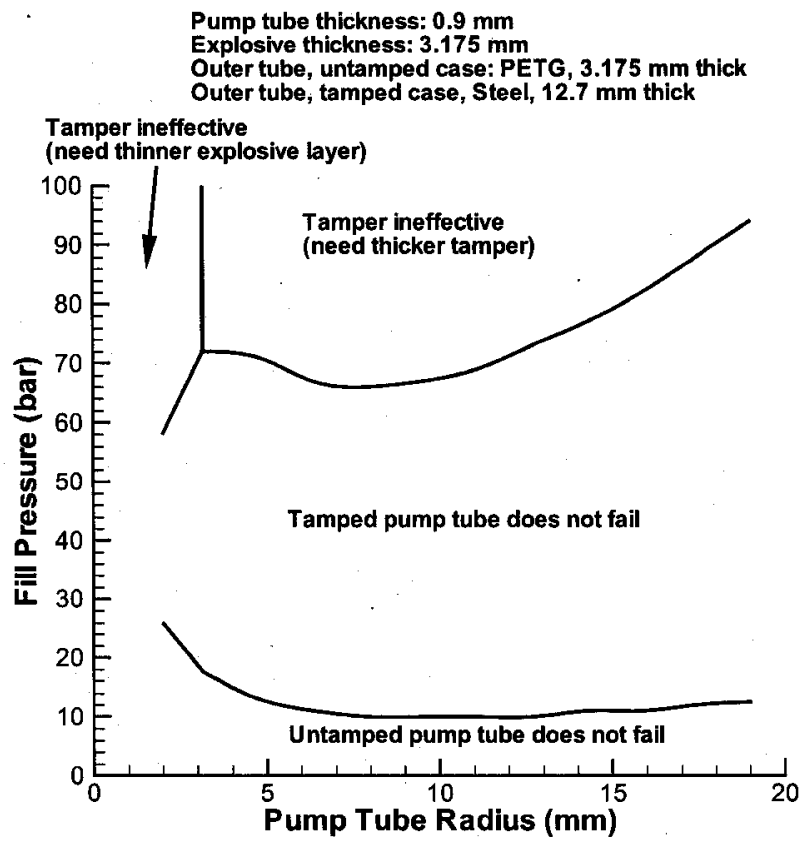

(a) Fill pressure vs pump tube radius

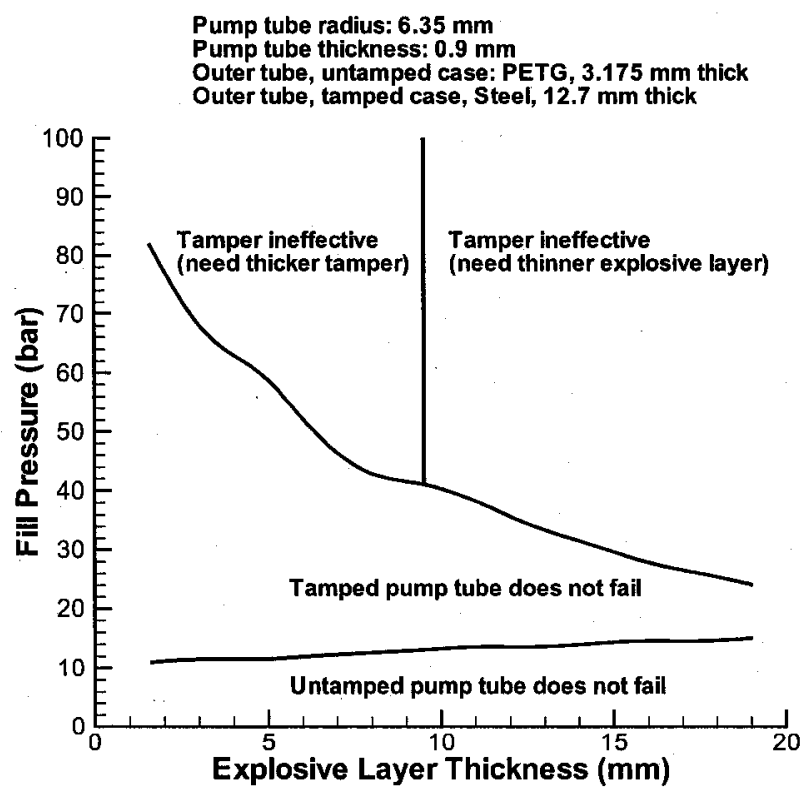

(b) Fill pressure $v s$ explosive layer thickness

Figure 2-7: Parameter maps 
increasing the explosive layer thickness helps in the absence of a tamper. On the other hand, a thinner layer is better when using a tamper. This is reasonable since a thicker explosive layer means the effect of the outer boundary will be felt at a later time.

\subsubsection{Standoff Calculation}

Measuring expansion histories experimentally is very challenging, especially if a tamper is used. What are usually measured experimentally are the shock speed and the distance separating the shock and the detonation at a certain point down the tube. If we can use the model developed in section 2.1 to predict this distance, which we call here the standoff distance, based on tube expansion, we would have some experimental data to validate the model predictions.

We start by considering a length of pump tube, as shown in figure $2-8(a)$. The tube is filled with driver gas at an initial pressure, density, and temperature $p_{0}, \rho_{0}$, and $T_{0}$. The piston is initially at $x=0$ and we are interested in the cross section at $x_{0}$. In a pump tube experiment, $x_{0}$ is where the diagnostic sensors to measure shock speed and standoff distance would be located. Once the piston starts moving at detonation velocity $U_{D}$, it will push a shock wave in front of it with speed $U_{s}$. As long as the shock wave does not go beyond $x_{0}$ and there is no leakage past the piston, the mass of driver gas between the piston and $x_{0}$ will be constant. When the shock reaches $x_{0}$, the totality of this gas will be in the shocked state, $p_{s}, \rho_{s}$, and $T_{s}$, as shown in figure $2-8(\mathrm{~b})$. This state can be calculated with normal shock relations. If we assume the tube walls are rigid, it is then easy to calculate what the standoff is:

$$
\begin{gathered}
\rho_{0} V_{0}=\rho_{s} V_{s} \\
\rho_{0} A x_{0}=\rho_{s} A x_{\text {standoff }}
\end{gathered}
$$




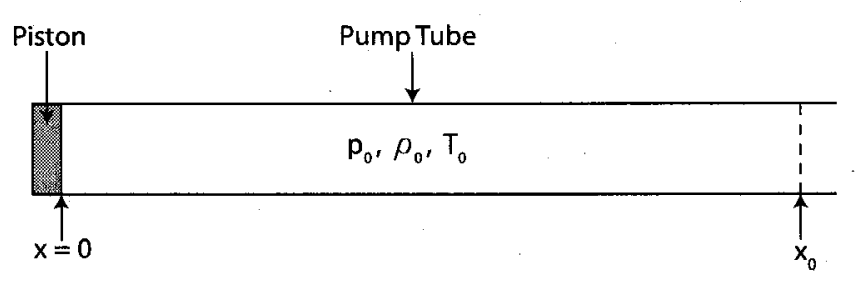

(a) Initial configuration

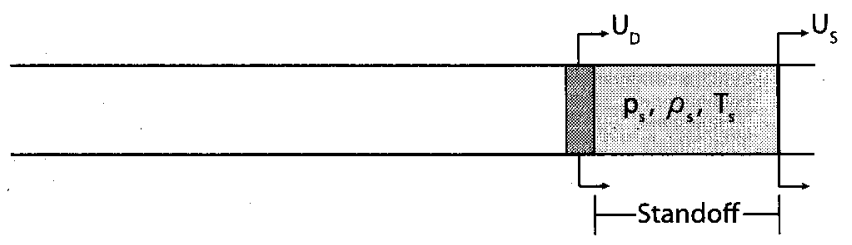

(b) Shock reaches $x_{0}$ - rigid tube

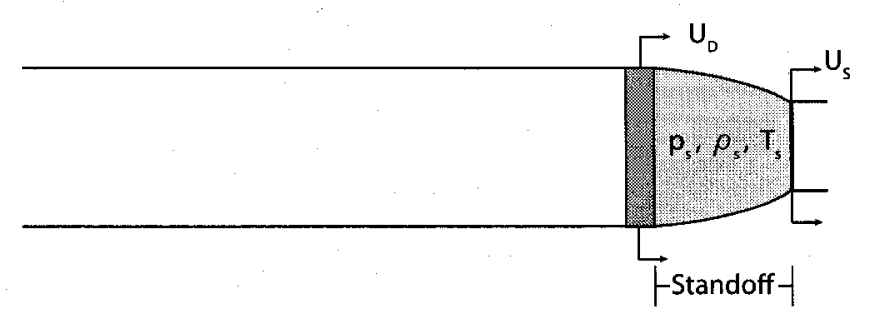

(c) Shock reaches $x_{0}$ - expanding tube

Figure 2-8: Volume of driver gas contained in the pump tube

$$
x_{\text {standoff }}=\frac{\rho_{0} x_{0}}{\rho_{s}}
$$

where $V$ is the volume of the driver gas and $A$ is the cross-sectional area of the pump tube. If the walls are not rigid and the tube expands, the result will be a reduction of the standoff, as shown in figure 2-8(c). Assuming a perfect piston and constant shock velocity, the volumes of shocked gas in figures 2-8(b) and 2-8(c) are the same. Then if we are capable of generating an expansion profile for the pump tube, we will be able to calculate what the standoff should be. 


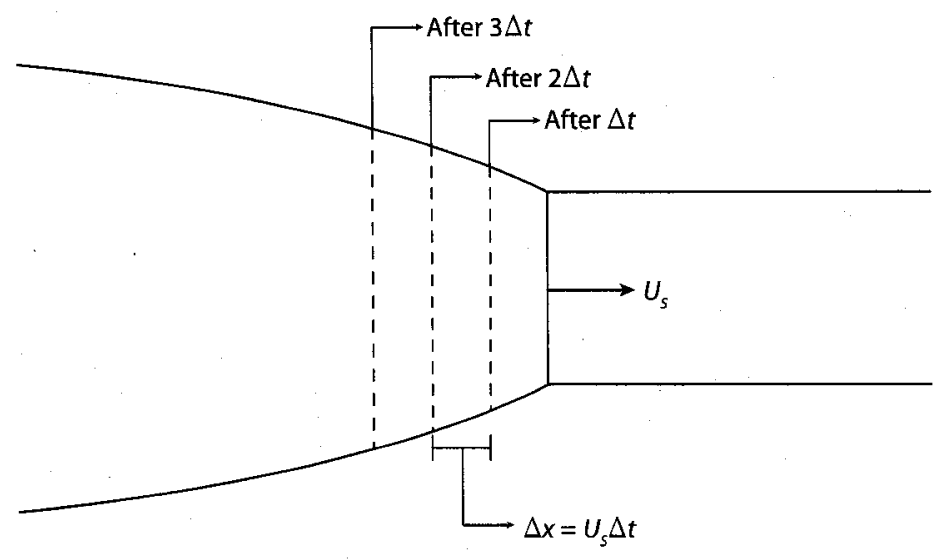

Figure 2-9: Converting time to distance

The goal thus far has been to obtain the pump tube radius as a function of time. In order to calculate the volume of the shocked gas inside the pump tube, we need to calculate the radius as a function of position. We perform the following coordinate transformation, referring to figure 2-9. At some time $t$ the shock is at a certain point $x$ down the pump tube. After a time $\Delta t$, it will have moved past this point by a distance $\Delta x$. Since the shock travels at shock velocity $U_{s}, \Delta x=U_{s} \Delta t$. Applying this transformation to our radius vs time profiles, we can now obtain radius vs length profiles. This means we can calculate the volume of shocked gas for the expanding tube. Note that we have to use the internal radius of the pump tube to calculate the volume of gas. Since we know that the volume should be the same as in the rigid tube case, we can stop the calculation once we have reached this volume and have the code return the value of the standoff distance.

Figure 2-10 shows the calculated standoff distances for a pump tube having an outer radius of $3.175 \mathrm{~mm}$, a thickness of $0.9 \mathrm{~mm}$, an explosive layer thickness of $4.75 \mathrm{~mm}$, and having a PETG plastic outer tube with a thickness of $3.15 \mathrm{~mm}$. The values of standoff are taken at $52 \mathrm{~cm}$ down the pump tube. The pressure is varied 


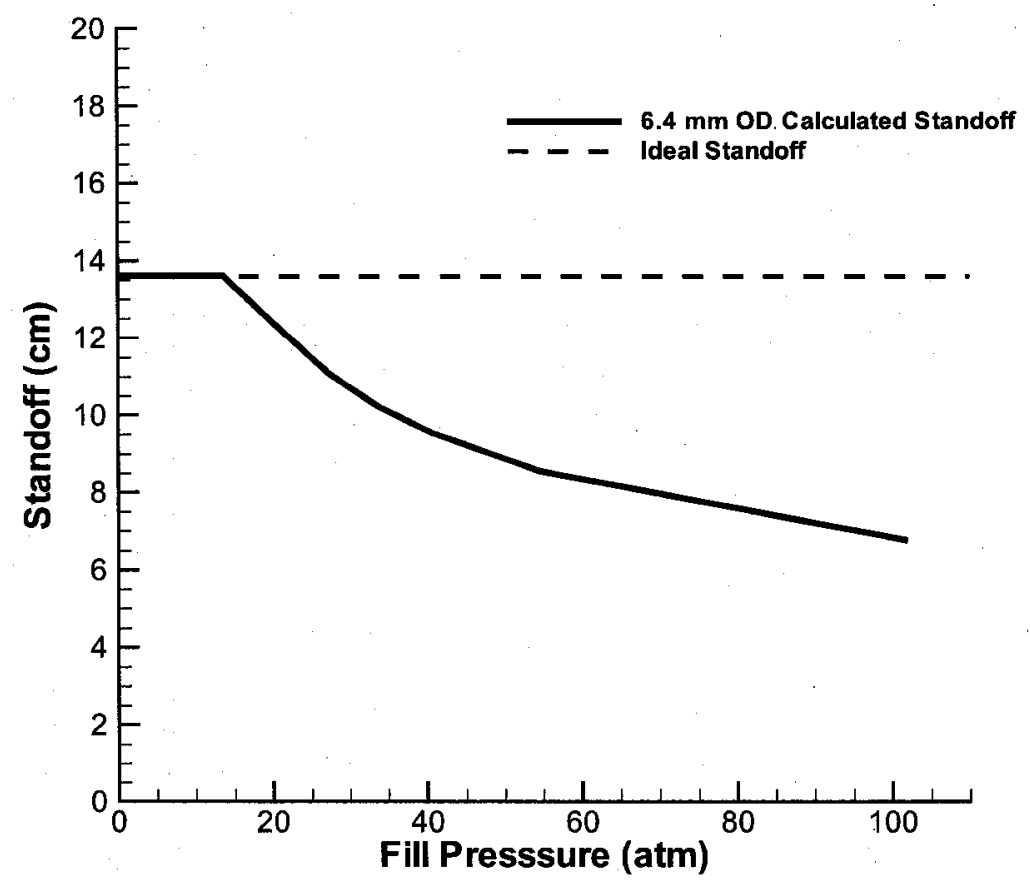

Figure 2-10: Calculated standoff distances at $52 \mathrm{~cm}$ from the initial collapse point for a $3.175 \mathrm{~mm}$ radius pump tube as a function of fill pressure 
from 10 to $100 \mathrm{~atm}$. The code was run until the necessary volume of shocked gas was reached, regardless of the elongation of the pump tube. The graph shows that, up to an initial fill pressure of about $13 \mathrm{~atm}$, pump tube expansion is negligible and we recover the ideal standoff. Raising the initial fill pressure beyond that results in pump tube expansion and a decrease in standoff distance. These predictions will be compared to experimental results in the next chapter. 


\section{CHAPTER 3 \\ Driver Experiments}

As was mentioned in section 1.1.2, the performance of the linear explosive driver is influenced by a number of non-ideal effects. These are primarily the expansion of the pump tube, the development of a boundary layer, and the formation of a metal jet at the initial collapse point. The most important of these was assumed to be the expansion of the pump tube and a theoretical model was developed in the previous chapter in order to gauge its effects on the precursor shock. The results obtained from the model need to be compared to experimental data.

Experiments were performed to ascertain the dynamics of the precursor shock as a function of the initial fill pressure of the pump tube. It was expected that at relatively low gas fill pressures, the experimental values would be close to ideal, and, as we increased the pressure, increased expansion of the pump tube would lead to a slower precursor shock and a shorter standoff distance. Different sizes of pump tubes were also investigated, and the effect of adding a tamper was also examined.

\section{$3.1 \quad 0.64 \mathrm{~cm}$ Outer Diameter Pump Tube Series}

\subsubsection{Experimental Setup}

The schematic of the apparatus used in the first series of driver experiments is shown in figure $3-1$. The pump tube is a $0.64 \mathrm{~cm}$ outer diameter, $0.46 \mathrm{~cm}$ inner diameter, stainless steel (304) tube. The outer tube is a PETG plastic tube with $1.59 \mathrm{~cm}$ inner diameter and $2.22 \mathrm{~cm}$ outer diameter. This leaves a thickness of $0.48 \mathrm{~cm}$ for the explosive. The explosive is nitromethane sensitized with $10 \%$ diethylenetriamine, 


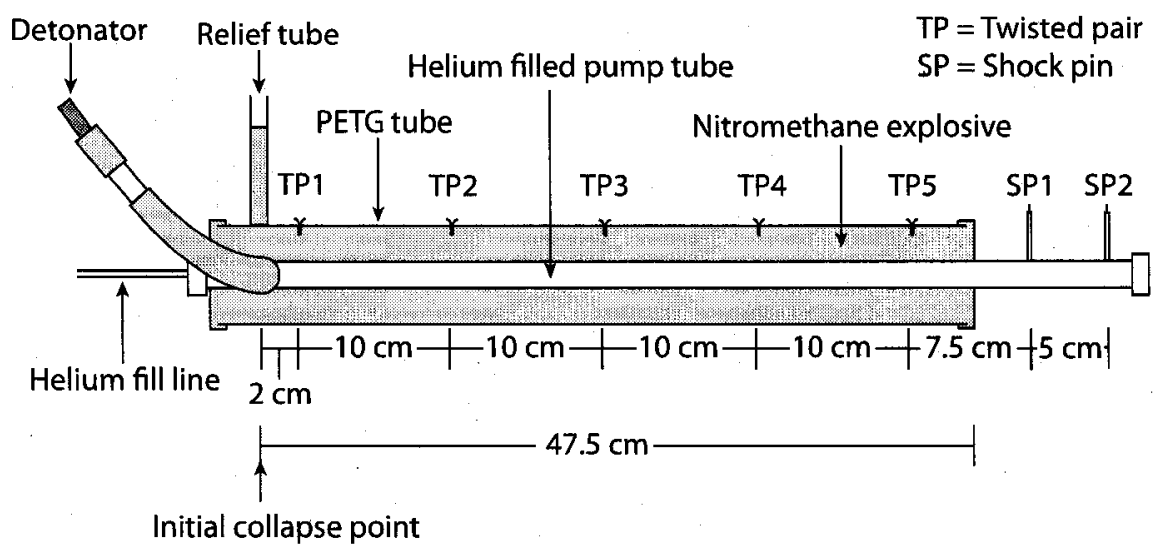

Figure 3-1: Schematic of the experimental apparatus used in the pressure variation series

which has a detonation velocity of $6 \mathrm{~km} / \mathrm{s}$. In helium, an ideal piston traveling at this velocity drives a $8.1 \mathrm{~km} / \mathrm{s}$ shock ahead of it.

Note that the detonator is inserted in a tube which bifurcates and enters the outer tube from either side. The tube is collapsed over a length of $47.5 \mathrm{~cm}$ from the point where the detonation enters the outer tube. The outer tube is instrumented with self-shorting twisted wire pair gauges. These gauges short when the detonation passes over them and send a signal to the oscilloscope, allowing us to track the detonation wave. The position of the detonation wave is assumed to be the same as the position of the virtual piston. There is a length of pump tube which extends outside the explosives. This length is instrumented with piezoelectric shock pins (Dynasen CA-1135). The shock pins record the arrival of the precursor shock wave, allowing us to measure shock velocity and standoff distance with the detonation. Holes are drilled in the pump tube at the shock pin locations, which are $49.5 \mathrm{~cm}$ and $54.5 \mathrm{~cm}$ away from the initial collapse point. The shock pins are inserted in these holes such that they are flush with the inner wall of the tube. The shock pins are 


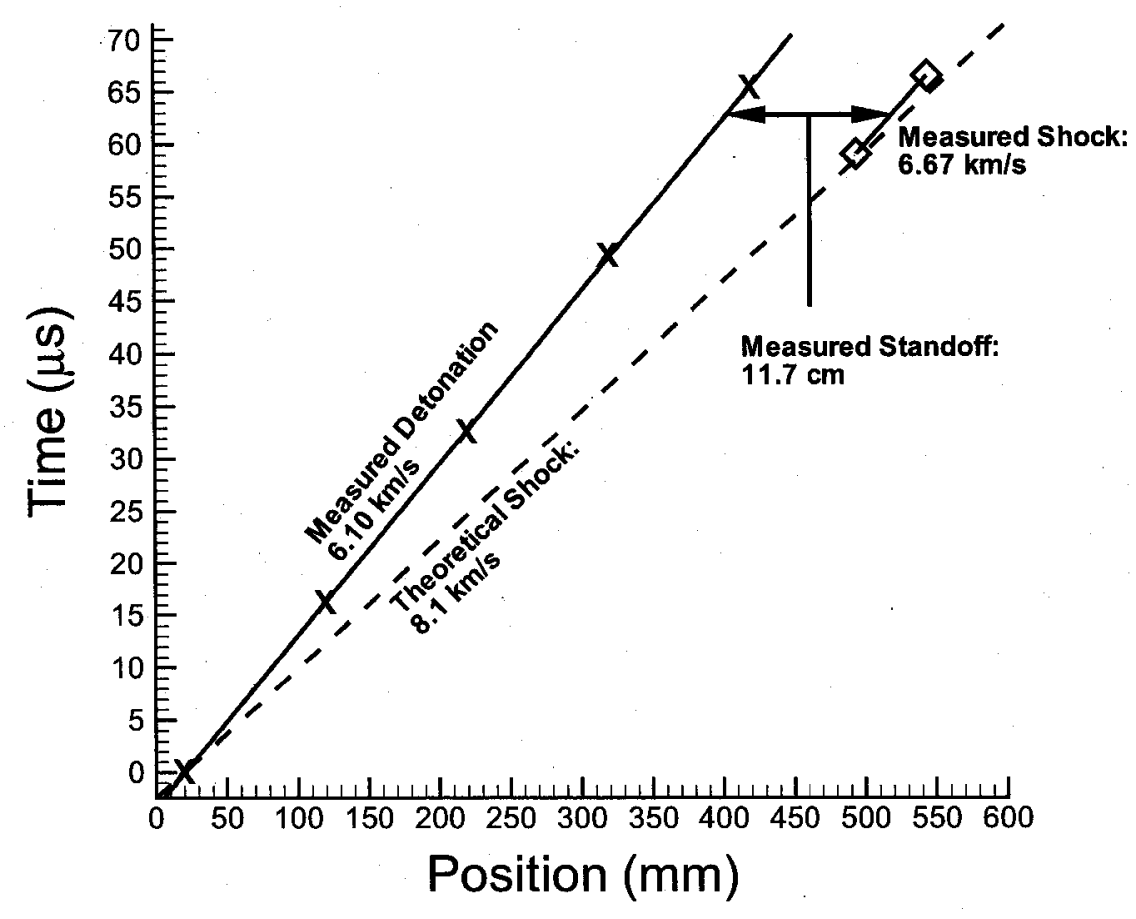

Figure 3-2: Position-time graph for 27.2 atm initial fill pressure

therefore in direct contact with the driver gas, which is helium, but do not create an obstacle for the shock wave. The end of the pump tube is capped.

\subsubsection{Results}

Experiments were carried out for initial fill pressures in the range of 10 atm to $100 \mathrm{~atm}$, keeping all other measurements constant. Trajectories of the detonation and the precursor shock were recorded by the twisted pairs and the shock pins, respectively. It is most useful to display the obtained data in the form of an $x-t$ graph. See figure 3-2 for a typical $x-t$ graph obtained from experiments in this series.

For each of the pressures investigated, the detonation velocity, shock velocity, and standoff distance between the shock and the detonation were measured. The 
Table 3-1: Summary of driver experiments using $0.64 \mathrm{~cm}$ outer diameter pump tubes

\begin{tabular}{|c|c|c|c|c|}
\hline $\begin{array}{l}\text { Shot } \\
\text { No. }\end{array}$ & $\begin{array}{c}\text { Fill } \\
\text { Pressure } \\
\text { (atm) }\end{array}$ & $\begin{array}{l}\text { Shock } \\
\text { Velocity } \\
(\mathrm{km} / \mathrm{s})\end{array}$ & $\begin{array}{c}\text { Standoff } \\
(\mathrm{cm})\end{array}$ & Comments \\
\hline D000 & 13.6 & - & - & Bad trigger. \\
\hline Do01 & 13.6 & 7.58 & 15.6 & Repeat of shot D000. \\
\hline $\mathrm{D} 002$ & 102.0 & 6.10 & 5.9 & \\
\hline D003 & - & - & - & No data \\
\hline D004 & 34.0 & 6.68 & 10.6 & \\
\hline D005 & 27.2 & 6.67 & 11.7 & \\
\hline$\overline{\mathrm{D}} 006$ & 40.8 & 6.65 & 10.3 & $\begin{array}{l}\text { No data obtained from the last } \\
\text { three twisted pairs. }\end{array}$ \\
\hline D007 & 51.0 & - & - & $\begin{array}{l}\text { No data obtained from the } \\
\text { shock pins. }\end{array}$ \\
\hline D008 & 54.4 & - & - & $\begin{array}{l}\text { No data obtained from the first } \\
\text { shock pin. }\end{array}$ \\
\hline D009 & 20.4 & 6.97 & 13.5 & $\begin{array}{l}\text { A tertiary shock pin glued to } \\
\text { the surface of the pump tube } \\
\text { was added between the other } \\
\text { two. }\end{array}$ \\
\hline D010 & 54.4 & 6.44 & 8.1 & $\begin{array}{l}\text { A tertiary shock pin was used } \\
\text { as in shot D009. }\end{array}$ \\
\hline$\overline{\mathrm{D} 011}$ & 9.5 & 7.81 & 18.2 & $\begin{array}{l}\text { Only one of the five twisted } \\
\text { pairs gave a signal. Deto- } \\
\text { nation trajectory was extrap- } \\
\text { olated from this single data } \\
\text { point. }\end{array}$ \\
\hline
\end{tabular}

shock velocity was computed from the shock pin data. The standoff distance was evaluated graphically at a distance of $52 \mathrm{~cm}$ away from the initial collapse point, which is the midpoint between both shock pins. This is illustrated in figure 3-2. In this manner we can obtain a value of shock velocity and standoff distance at this point for each pressure investigated.

The details of the individual experiments performed in this series are given in table 3-1. The results are shown in graphical form in figure 3-3. 


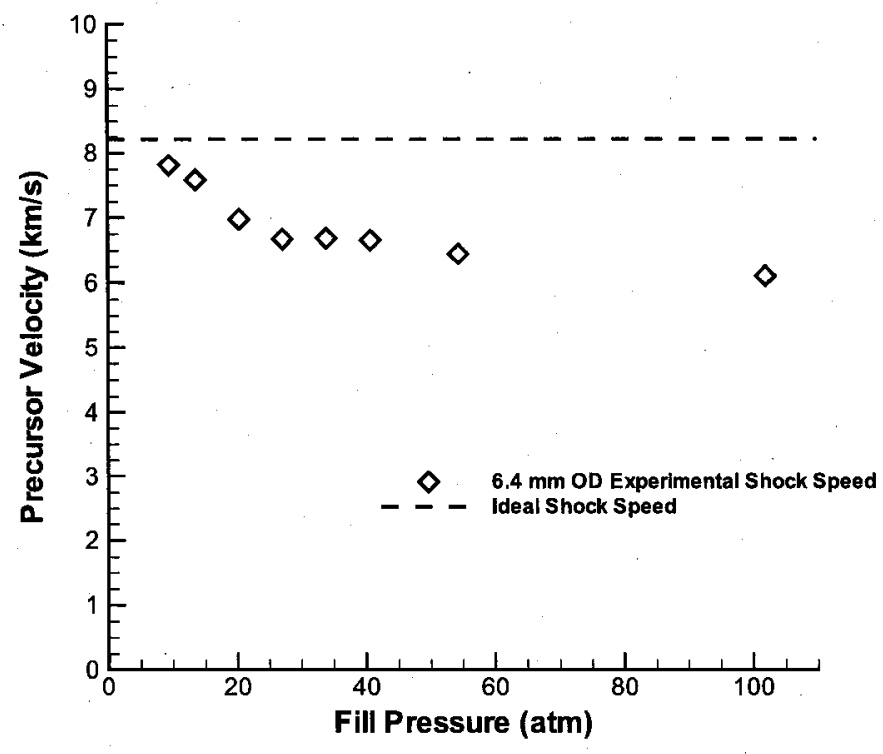

(a) Precursor shock velocity $v s$ fill pressure

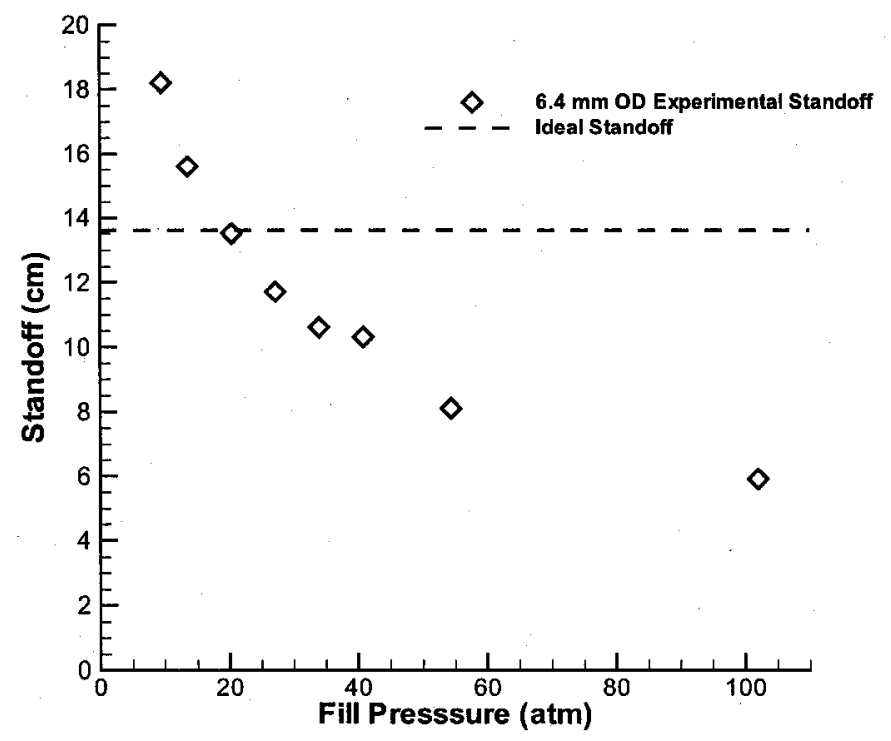

(b) Precursor shock standoff $v s$ fill pressure

Figure 3-3: Precursor shock velocity and standoff vs initial fill pressure for a $0.64 \mathrm{~cm}$ outer diameter pump tube. Measurements made $52 \mathrm{~cm}$ away from the initial collapse point. 
First we note that the velocity and standoff decrease as we increase fill pressure, as was expected. Next, we notice that for 10 atm fill pressure, the standoff is greater yet the velocity is smaller than they are in the ideal case $(6.0 \mathrm{~km} / \mathrm{s}$ piston driving a $8.1 \mathrm{~km} / \mathrm{s}$ precursor shock). This suggests the presence of a shaped charge effect caused by the initial collapse of the tube. It also indicates that the shock velocity is not constant but rather it decays as it progresses down the tube. The shaped charge effect should be avoided since the metal jet generated may damage the projectile and mix heavy metal particles to the driver gas, thus increasing its acoustic impedance.

Some difficulties were encountered when inserting the shock pins into the pump tube and sealing them properly to resist the fill pressure. Such an arrangement is fragile, and leaks and breaks were not uncommon. Attempts were made to determine whether the shock pins could record the passage of the shock when mounted on the outside surface of the pump tube and, if so, to estimate the delay caused by measuring through the tube wall. For shots D009 and D010, a third shock pin was added between the regular two, at $52 \mathrm{~cm}$ from the initial collapse point. This third shock pin was attached directly to the surface of the tube rather than inserted in a hole in the pump tube. The signals given by these shock pins were not as sharp at the ones in direct contact with the driver gas, but were clear enough to give times of arrival of the shock. Shock arrival data obtained from these shock pins was only 0.4 to $0.6 \mu$ s late, which is acceptable for the purposes of these measurements. Mounting the shock pins on the outside of a thin-walled tube (about $0.9 \mathrm{~mm}$ thick in this case) is therefore acceptable and facilitates the construction of these charges.

The theoretical detonation velocity for nitromethane is $6.0 \mathrm{~km} / \mathrm{s}$. The detonation velocities measured were in the range of $6.1 \pm 0.04 \mathrm{~km} / \mathrm{s}$. This represents experimental errors in the range of $1-2.3 \%$. Some trouble with the twisted pairs 


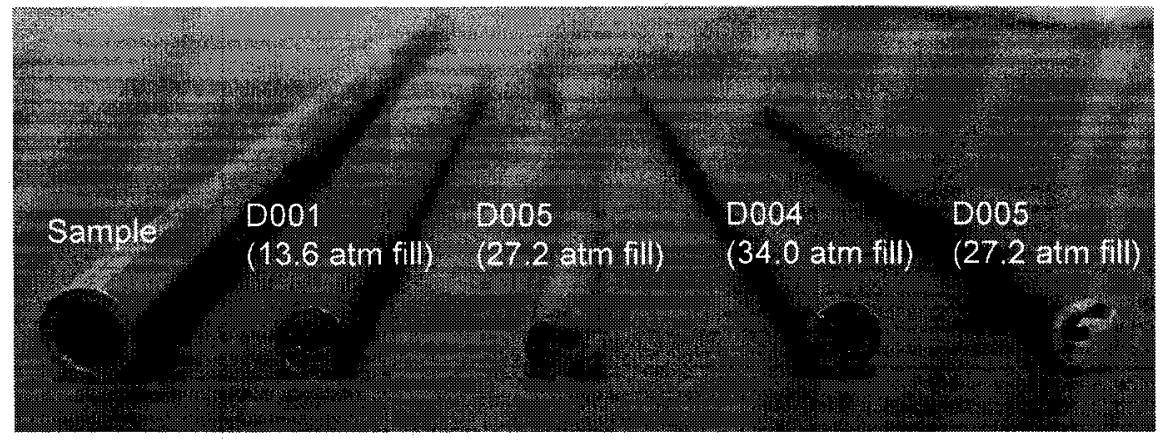

Figure 3-4: Photograph of cross-sectioned recovered pump tubes showing incomplete collapse. The cross sections are about $15 \mathrm{~cm}$ from the initial collapse point.

shorting during shot setup led to a new arrangement for these probes. Rather than drilling a hole into the outer tube and dipping the twisted pairs into the nitromethane explosive, the twisted pairs were glued to the surface of the tube and a thin metal plate was fixed on top of it. In this manner, the twisted pairs are not shorted directly by the detonation, but rather by being squeezed onto the metal plate by the detonation.

Recovered collapsed pump tubes were sectioned about $15 \mathrm{~cm}$ from the initial collapse point to verify whether they had been completely collapsed or not. Figure 34 shows that a small hole remains at the center of the tube. This hole is larger for higher initial fill pressures. The recovered tubes for shots under 25 atm initial fill pressure are relatively straight and intact. However, as we raise the initial fill pressure beyond that point, they begin showing signs of rupture along their lengths, as shown in figure 3-5, especially as we get further from the initial collapse point. For the highest pressure shots (above $50 \mathrm{~atm}$ ), the recovered tubes are shredded at one end, and we often cannot recover the entire length of collapsed tube, as shown in figure 3-6. Perhaps increasing the wall thickness could help contain pressure longer and prevent the tube from ripping. 


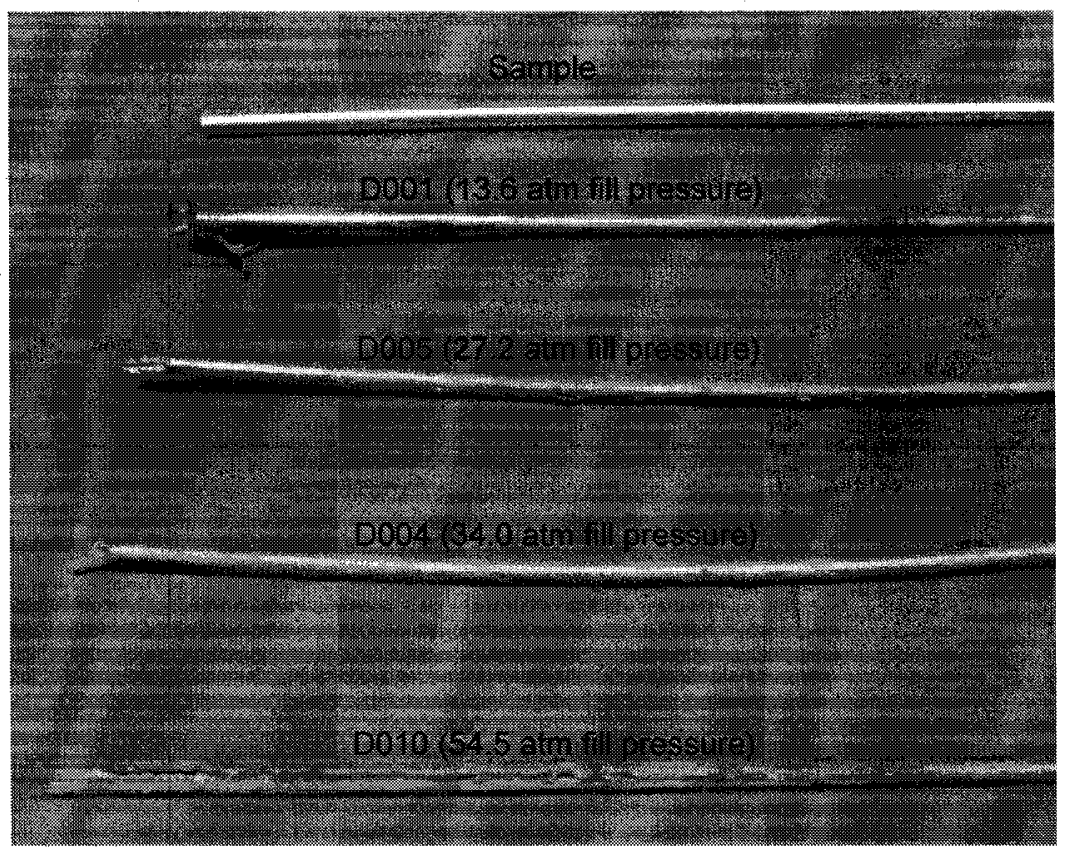

Figure 3-5: Photograph of recovered pump tubes showing rupturing along the length of the tubes for the higher pressure shots

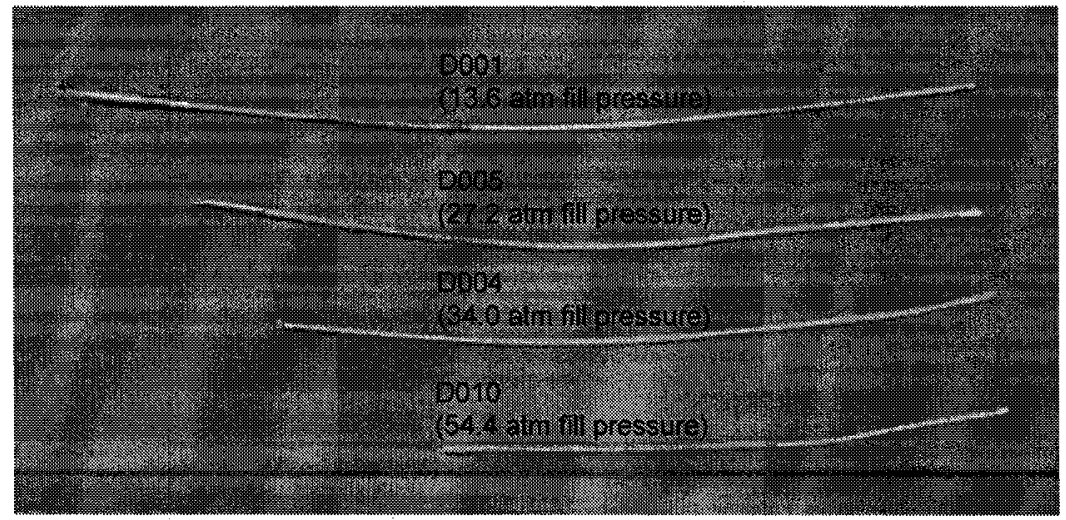

Figure 3-6: Photograph of recovered pump tubes showing that total recovered length of the tube tends to decrease as we increase initial fill pressure 


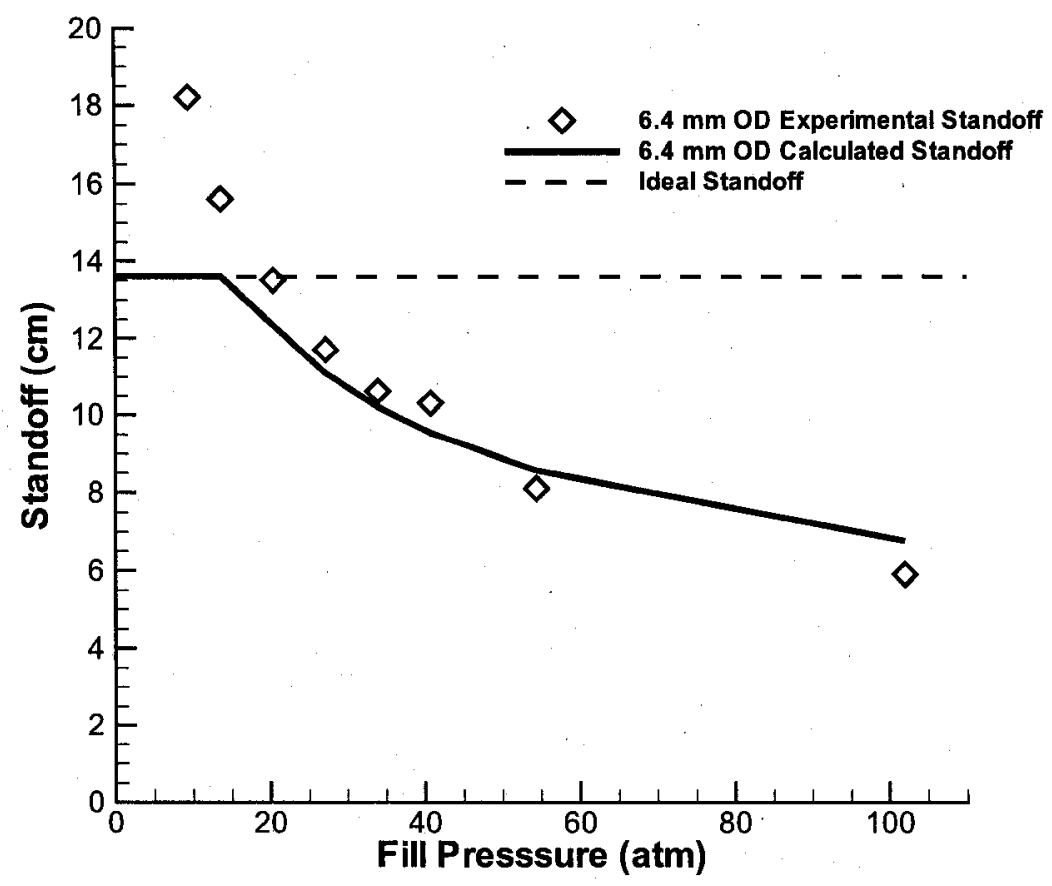

Figure 3-7: Calculated standoff distances for a $3.175 \mathrm{~mm}$ radius pump tube as a function of fill pressure, compared to experimental results

\subsubsection{Comparison with Expanding Tube Model}

The expanding tube model presented in Chapter 2 was used to calculate the standoff distance for the driver measurements used in this first series of driver experiments. The results obtained from the model are shown in figure $2-10$ in section 2.2.2. Figure 3--7 compares the experimental values to the calculated values of standoff for this series.

The model and experimental trends agree very well. In an experiment, there is the shaped charge effect (as discussed in section 1.1.2) which can initially overdrive the shock wave. This effect is more prominent at lower pressures, which is why values that exceed the ideal value of standoff are obtained experimentally at lower pressures. 
It is worth reiterating that the computation was run until the necessary volume of shocked gas was reached (refer to section 2.2.2), regardless of the elongation of the pump tube. In other words, tube bursting was not considered in the computation. It is likely that, for the higher pressures shown here, the pump tube failed during the experiment, which would bring down the recorded values of standoff.

\section{$3.2 \quad 0.95 \mathrm{~cm}$ Outer Diameter Pump Tube Series}

Since the recovered $0.64 \mathrm{~cm}$ outer diameter pump tubes showed signs of failing at higher pressures, the wall thickness was increased for this series of driver experiments in hopes that a thicker pump tube would survive longer at higher pressures. The experimental setup is very similar to the previous one. All shots in this series were made at 34 atm initial fill pressure.

For the first shot (D012), the pump tube was a $0.95 \mathrm{~cm}$ outer diameter, $0.43 \mathrm{~cm}$ inner diameter tube. Note here that the inner diameter is roughly the same as that of the $0.64 \mathrm{~cm}$ pump tube. The shock pins were inserted in bores made in the tube such that there was a thickness of only about $0.4-0.5 \mathrm{~mm}$ of steel between the shock pins and the driver gas. The outer tube was a PETG plastic tube with $2.54 \mathrm{~cm}$ outer diameter and $2.22 \mathrm{~cm}$ inner diameter. This left a thickness of $0.64 \mathrm{~cm}$ for the explosive. However, this proved to be too thick a pump tube to properly implode with this explosive thickness. The next shot (D013) used a $0.95 \mathrm{~cm}$ outer diameter, $0.63 \mathrm{~cm}$ inner diameter tube, reducing the wall thickness from $2.41 \mathrm{~cm}$ to $1.65 \mathrm{~cm}$. This shot gave a very low shock velocity.

The early launchers used the same size of pump tube and outer tube as shot D013. Two of these (L002 and L003) were instrumented with shock pins to record the shock trajectory, effectively making them combined driver and launcher experiments. The shock data obtained from shot L002 agreed well with theory. Shot L003 had shock 
Table 3-2: Summary of driver experiments using $0.95 \mathrm{~cm}$ outer diameter pump tubes. All shots done at 34 atm initial fill pressure.

\begin{tabular}{|c|c|c|l|}
\hline $\begin{array}{c}\text { Shot } \\
\text { No. }\end{array}$ & $\begin{array}{c}\text { Shock } \\
\text { Velocity } \\
(\mathrm{km} / \mathrm{s})\end{array}$ & $\begin{array}{c}\text { Standoff } \\
(\mathrm{cm})\end{array}$ & \multicolumn{1}{|c|}{ Comments } \\
\hline D012 & - & - & $\begin{array}{l}\text { Wall thickness of 2.14 cm. Four shock } \\
\text { pins were used. Shock pins went out } \\
\text { of sequence, indicating that there may } \\
\text { not have been a precursor. Recov- } \\
\text { ered pump tube showed an incomplete } \\
\text { pinch. }\end{array}$ \\
\hline D013 & 3.64 & 9.9 & $\begin{array}{l}\text { Wall thickness decreased to 1.65 cm. } \\
\text { Two shock pins were used. Very low } \\
\text { shock velocity. }\end{array}$ \\
\hline L002 & 8.06 & 12.7 & $\begin{array}{l}\text { Three shock pins were used. Shock } \\
\text { speed measured at 45 cm from ini- } \\
\text { tial collapse point, rather than 52 cm. } \\
\text { Standoff extrapolated to } 52 \text { cm. }\end{array}$ \\
\hline L003 & 5.54 & 9.8 & $\begin{array}{l}\text { Shock trajectory recorded over a longer } \\
\text { distance by using more shock pins } \\
\text { spaced along the pump tube. }\end{array}$ \\
\hline
\end{tabular}

pins all along the length of the pump tube in order to record the shock trajectory over a longer distance. This data gave a low shock velocity at $52 \mathrm{~cm}$ from the initial collapse point which did not match either of the previous two shots.

The details of the individual experiments performed in this series are given in table 3-2. The data does not seem to be reproducible for this size of pump tube. Perhaps the tube wall is too thick for the explosive layer thickness used. If this is true, a tamper may help properly collapse the tube. Therefore, it was decided to proceed to a larger diameter and thinner walled pump tube surrounded by a tamper.

\section{$3.3 \quad 1.27 \mathrm{~cm}$ Outer Diameter Pump Tube Series}

Increasing the thickness of the pump tube did not appear to be an adequate strategy for increasing pump tube performance. The third series of driver experiments used pump tubes of the same thickness as the $0.64 \mathrm{~cm}$ pump tube series 


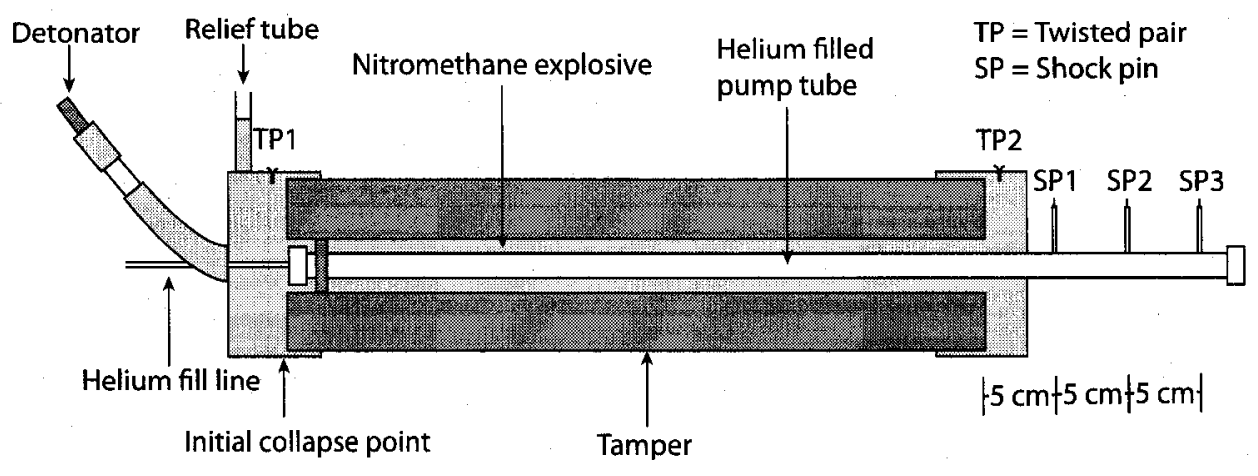

Figure 3-8: Typical setup for $1.27 \mathrm{~cm}$ outer diameter driver shot

$(0.9 \mathrm{~mm})$, but with a larger outer diameter: $1.27 \mathrm{~cm}$. Since we seemed to experience difficulty properly collapsing the $0.95 \mathrm{~cm}$ outer diameter pump tubes, this series of driver experiments used a tamper. The expansion histories computed by the expanding tube model discussed in Chapter 2 indicate that this may be a better strategy to prevent pump tube failure as well.

The following changes were made to the experimental setup. The twisted pairs were inserted in clear PVC plastic caps at either end of the tamper and were in direct contact with the nitromethane. The detonation was initiated from the rear rather than from either side, as it was in all the previous shots. This was done for two reasons: to avoid having to drill holes in the thick metal tamper and to help obtain a planar detonation wave for symmetric implosion of the pump tube. See figure 3-8 for a diagram of the typical setup for this series of driver experiments.

\subsubsection{Shot D014}

The first shot in this series (D014) used the PETG plastic outer tube from the $0.95 \mathrm{~cm}$ outer diameter pump tube shots, however this tube was then inserted into a $5.08 \mathrm{~cm}$ outer diameter and $2.54 \mathrm{~cm}$ inner diameter mild steel tube which acted as a tamper. Since the pump tube diameter was scaled up by a factor of two, the length of the pump tube was also increased by a factor of two, and the shock was 
measured at a distance of about $100 \mathrm{~cm}$ from the initial collapse point. Three shock pins were used, at $95 \mathrm{~cm}$, at $100 \mathrm{~cm}$ and at $105 \mathrm{~cm}$ from the initial collapse point. The detonation was measured by four twisted pairs, two at either end of the tamper. The initial fill pressure was 20.4 atm.

Both the measured shock $(8.53 \mathrm{~km} / \mathrm{s})$ and detonation $(6.47 \mathrm{~km} / \mathrm{s})$ speeds were rather high for this shot. Precompressing an explosive is capable of increasing the detonation velocity of the explosive [11]. It is possible that the expanding pump tube precompressed the nitromethane explosive against the tamper, which would explain the high detonation velocity. However, for the piston velocity and initial fill pressure here, the post shock pressure in the driver gas reaches about $190 \mathrm{MPa}$. The expanding tube model shows that the pump tube and tamper reach an equilibrium state where the nitromethane is at the post-shock pressure. According to [18], nitromethane compressed to $190 \mathrm{MPa}$ reaches a density of about $1.2 \mathrm{~g} / \mathrm{cc}$. This translates in a detonation velocity of about $6.2 \mathrm{~km} / \mathrm{s}$ according to [11]. This indicates that the precompression effect may be present in tamped driver shots, but not to the extent that data from this first shot seems to indicate. It is possible that the twisted pairs shorted prematurely due to a wave transmitted through the unreacted explosive from the precursor shock. The ideal shock speed for a $6.47 \mathrm{~km} / \mathrm{s}$ piston is $8.75 \mathrm{~km} / \mathrm{s}$, which is above the measured value. The measured standoff was about $26 \mathrm{~cm}$, which is close to the ideal value, whether using the accepted detonation speed in nitromethane $(6.00 \mathrm{~km} / \mathrm{s})$ or the measured value. The recovered pump tube measured about $80 \mathrm{~cm}$ and was completely collapsed initially. It was ruptured along its entire length, and the damage worsened farther away from the initial collapse point, as shown in figure 3-9. 


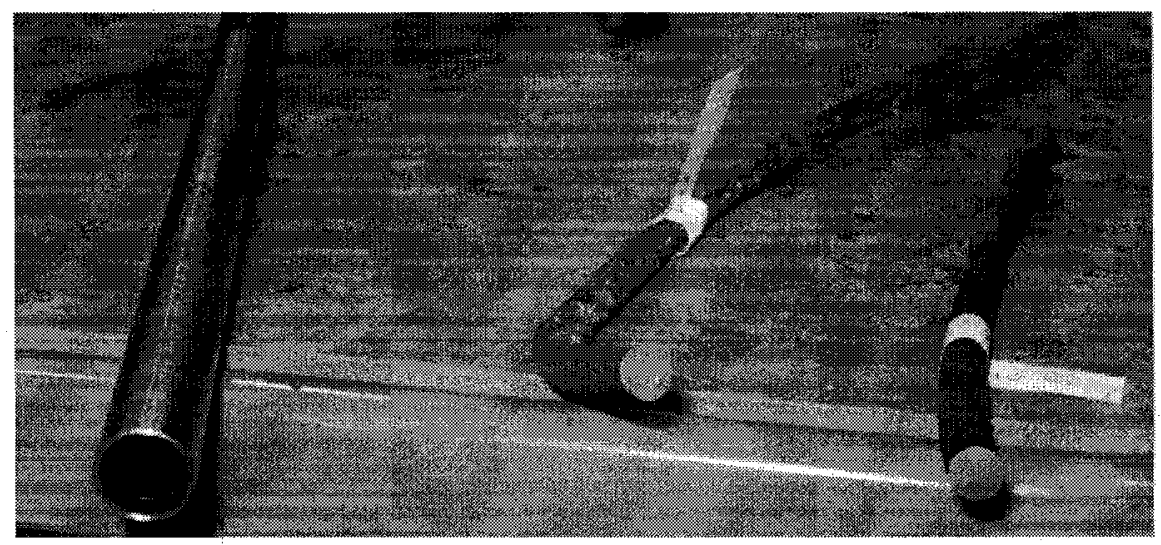

Figure 3-9: Photograph of the recovered pump tube for shot D014 showing a complete collapse initially and rupturing along most of its length

\subsubsection{Shot D015}

The second shot of the series (D015) eliminated the PETG plastic tube altogether and used a $1.91 \mathrm{~cm}$ inner diameter, $4.45 \mathrm{~cm}$ outer diameter mild steel tamper only. The shock was measured at about $80 \mathrm{~cm}$ from the initial collapse point. Due to human error prior to the shot, the exact pressure at which this shot was done is not known. We estimate it is in the range of 50 to $70 \mathrm{~atm}$.

Despite the high initial fill pressure, the precursor velocity was measured to be about $7.89 \mathrm{~km} / \mathrm{s}$, whereas it had fallen to about $6.5 \mathrm{~km} / \mathrm{s}$ for the $0.64 \mathrm{~cm}$ pump tube series at these pressures. The detonation velocity was $6.57 \mathrm{~km} / \mathrm{s}$, which is slightly higher than for shot D014 and supports the precompression hypothesis. The post shock pressure in this case lies between 400 and $560 \mathrm{MPa}$. This translates into a theoretical detonation velocity in the range of 6.4 to $6.5 \mathrm{~km} / \mathrm{s}$ due to precompression $[11,18]$. A piston traveling at $6.57 \mathrm{~km} / \mathrm{s}$ would generate an $8.88 \mathrm{~km} / \mathrm{s}$ shock. The recorded standoff was about $14 \mathrm{~cm}$, whereas the ideal standoff is about $21 \mathrm{~cm}$, using either a $6.00 \mathrm{~km} / \mathrm{s}$ or a $6.57 \mathrm{~km} / \mathrm{s}$ piston. 


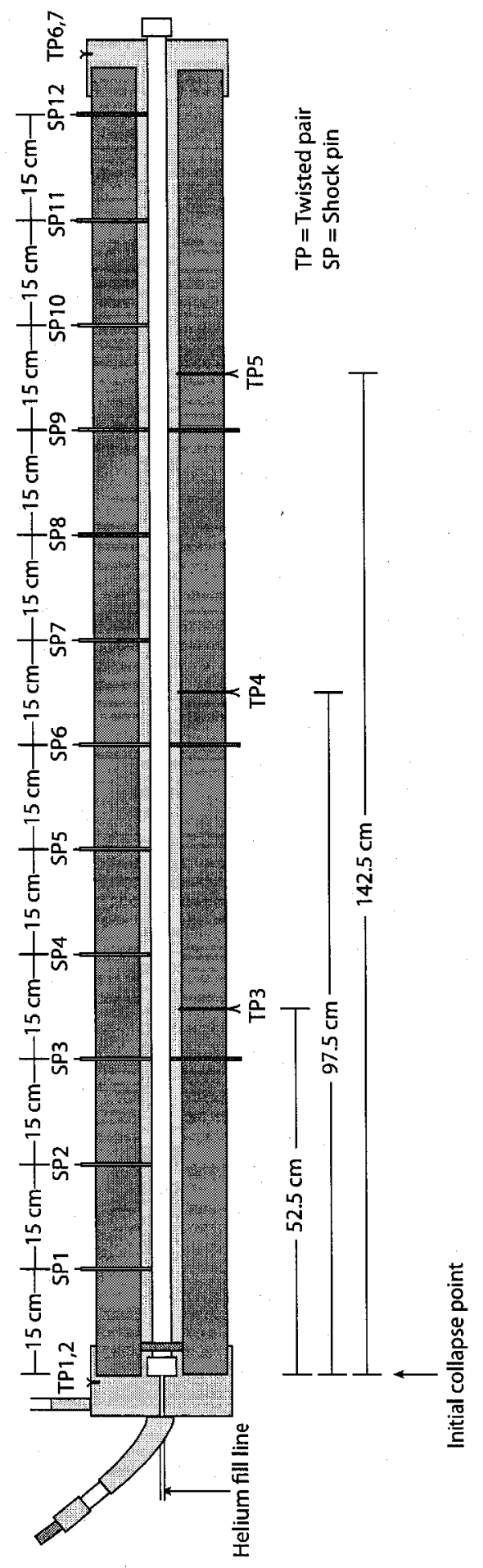

Figure 3-10: Experimental setup for a $1.8 \mathrm{~m}$ long driver shot 


\subsubsection{Shot D016}

We know that the precursor shock slows down as it travels down the pump tube. The third shot of this series (D016) was a $1.8 \mathrm{~m}$ long driver. Holes were drilled in the tamper so that shock pins could be inserted inside and rest against the pump tube. The shot was instrumented with shock pins at $15 \mathrm{~cm}$ intervals along its entire length so that the entire shock trajectory could be tracked. Some holes were drilled into the tamper to allow twisted pairs to be inserted into the nitromethane to measure detonation velocity as well. See figure 3-10 for a diagram of this shot. It used the same pump tube and tamper dimensions as shot D015. The fill pressure was 20.4 atm.

The last two shock pins gave no clear signal, so the shock was measured up to $1.5 \mathrm{~m}$ away from the initial collapse point. The shock pin data indicated that the precursor shock wave was initially traveling at a speed of about $9.96 \mathrm{~km} / \mathrm{s}$ and decayed up to $6.92 \mathrm{~km} / \mathrm{s}$ when it had traveled $1.5 \mathrm{~m}$. The twisted pairs inside the tamper gave questionable data. They showed the detonation oscillating between 5.8 and $7.7 \mathrm{~km} / \mathrm{s}$. It is probable the compression of the nitromethane as a result of tube expansion shorted the twisted pairs prematurely. The twisted pairs at either end of the tamper should not be affected by this and gave a detonation velocity of about $6.60 \mathrm{~km} / \mathrm{s}$, again higher than we would expect from precompression effects. A piston traveling at that speed would generate an $8.92 \mathrm{~km} / \mathrm{s}$ shock. Figure $3-11$ shows the $x-t$ diagram for shot D016. Shown in the figure are the measured shock and detonation waves, as well as the ideal shock trajectories for both $6.00 \mathrm{~km} / \mathrm{s}$ and $6.60 \mathrm{~km} / \mathrm{s}$ pistons. The measured shock trajectory falls almost completely between , these two cases. It appears that the shock wave forms late and is overdriven for a 


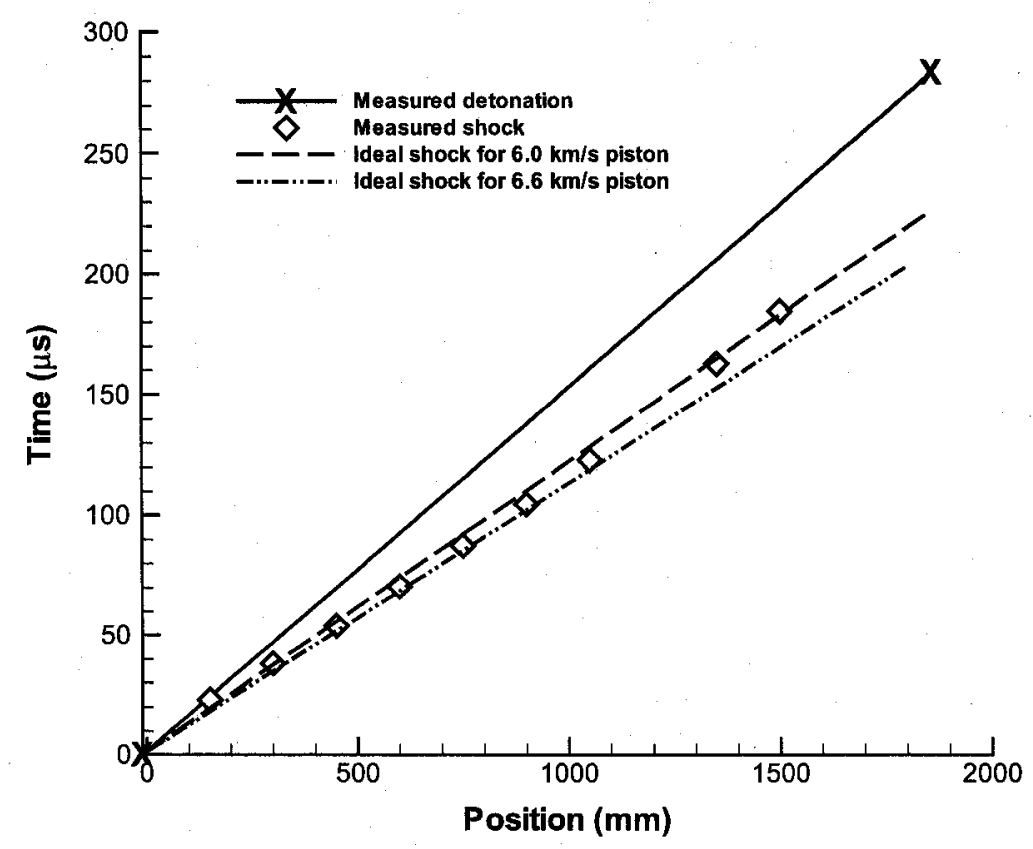

Figure 3-11: $x-t$ graph for a $1.8 \mathrm{~m}$ long driver with 20.4 atm initial fill pressure short period of time, after which its velocity decays slowly. The recovered pump tube measured about $90 \mathrm{~cm}$, the last $15 \mathrm{~cm}$ of which were highly damaged.

\subsubsection{Shot D017}

Shot D017 was a repeat of shot D016, but at an initial fill pressure of 68 atm. All other measurements and diagnostics remained the same. The shock pins show the initial shock speed was about $8.36 \mathrm{~km} / \mathrm{s}$. The shock then oscillates in the range of 7.15 to $9.23 \mathrm{~km} / \mathrm{s}$ throughout its trajectory. The expanding tube model shows that, for an initial fill pressure of $68 \mathrm{~atm}$, the pump tube bursts after about $45 \mu \mathrm{s}$ of exposure to post-shock pressure (using the ideal $6.0 \mathrm{~km} / \mathrm{s}$ piston driving a $8.1 \mathrm{~km} / \mathrm{s}$ shock), and that the tamper used in this shot was not thick enough to prevent pump tube failure. It is probable that the pump tube failed in the experiment, which slowed down the shock and dropped the pressure inside the pump tube, enough for it to 
stop failing. This then allowed the pressure to build up once again and the shock to accelerate, until the pump tube failed again, and so on. This would explain the oscillating shock velocity observed here.

The twisted pairs recorded an event traveling in the range of 7.06 to $8.38 \mathrm{~km} / \mathrm{s}$. Even if we exclude the twisted pairs inside the tamper, the ones at the extremities recorded a velocity of $7.88 \mathrm{~km} / \mathrm{s}$. It is clear that.something else than the detonation wave shorted the twisted pairs, most probably the compression of the nitromethane as a result of tube expansion. This becomes evident when comparing the shock pin data to the twisted pair data in an $x-t$ diagram, as shown in figure 3-12. The twisted pair signals come very shortly after the shock pin signals, and both trajectories seem to match. This suggests that the twisted pairs recorded the shock, not the detonation. The recovered pump tube measured about $63 \mathrm{~cm}$, which is 27 $\mathrm{cm}$ shorter than for the $20.4 \mathrm{~atm}$ initial fill pressure shot. The last $15 \mathrm{~cm}$ of the recovered pump tube were highly damaged.

\subsubsection{Shot D018}

This shot used the same pump tube dimensions as the previous shots of this series, but we replaced the tamper by a PETG plastic tube. The explosive layer thickness was increased to about $1 \mathrm{~cm}$ to make sure the pump tube would be properly collapsed. The shock was measured at $123 \mathrm{~cm}$ by three shock pins. The fill pressure was $20.4 \mathrm{~atm}$, the same as shots D014 and D016.

Unfortunately, only one shock pin returned a signal. However, if we assume the shock originated at $x=0$, the average shock velocity using this single data point is about $8.64 \mathrm{~km} / \mathrm{s}$. This data point can also be compared to the $x-t$ data from shots D014 and D016. This is shown in figure 3-13. The expanding tube model predicts that, for an untamped $1 \mathrm{~cm}$ thick explosive layer and a 20.4 atm initial fill pressure, 


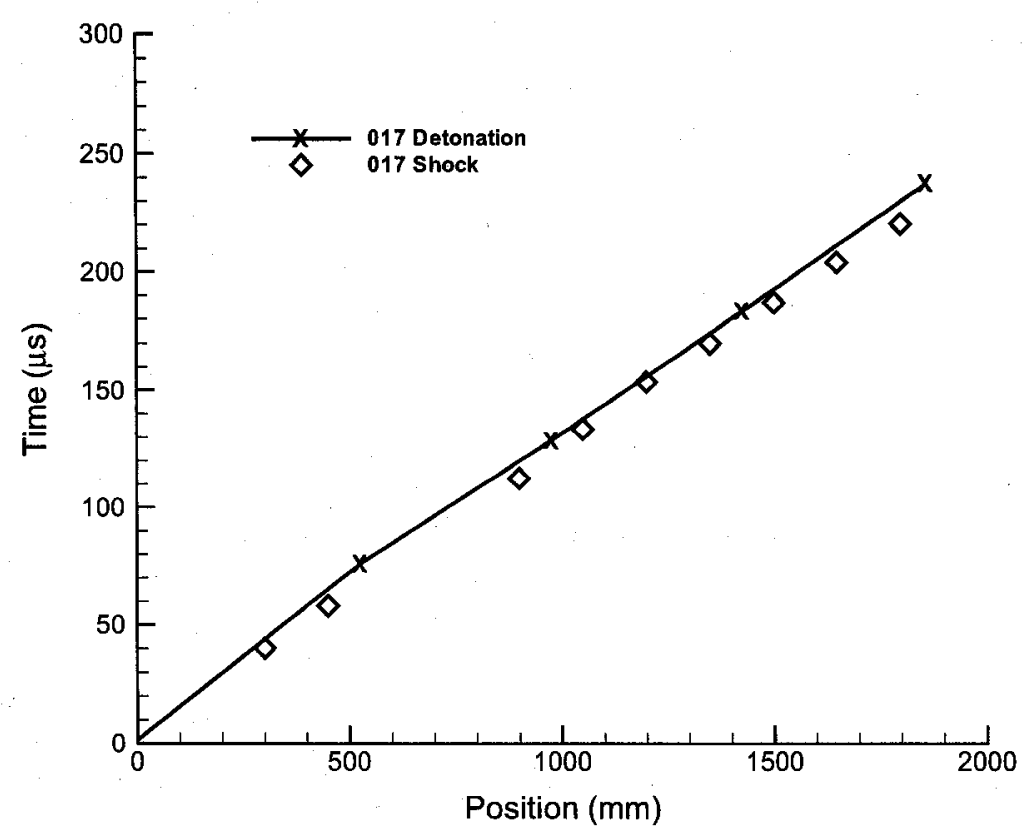

Figure 3-12: $x-t$ graph for a $1.8 \mathrm{~m}$ long driver with 68 atm initial fill pressure the pump tube will fail after about $30 \mu \mathrm{s}$. The data, however, suggests that the untamped version of the driver performs as well as the tamped version for this fill pressure. It should be noted that the amount of explosives used for this shot was several times that used for tamped shots.

The total length of pump tube recovered was about $40 \mathrm{~cm}$, which is about half the length recovered from the tamped shot at the same fill pressure (shot D014). The tube was well collapsed over the first $15 \mathrm{~cm}$, but the remaining length had a $2 \mathrm{~mm}$ hole along the center. Comparing the lengths recovered from shots D018 and D014, the untamped shot produced a more symmetric and complete collapse, and the pump tube shows less signs of rupture. The total recovered length is about half of what was recovered from the tamper shot. It seems tampered pump tubes survive longer, but do not pinch as uniformly. 


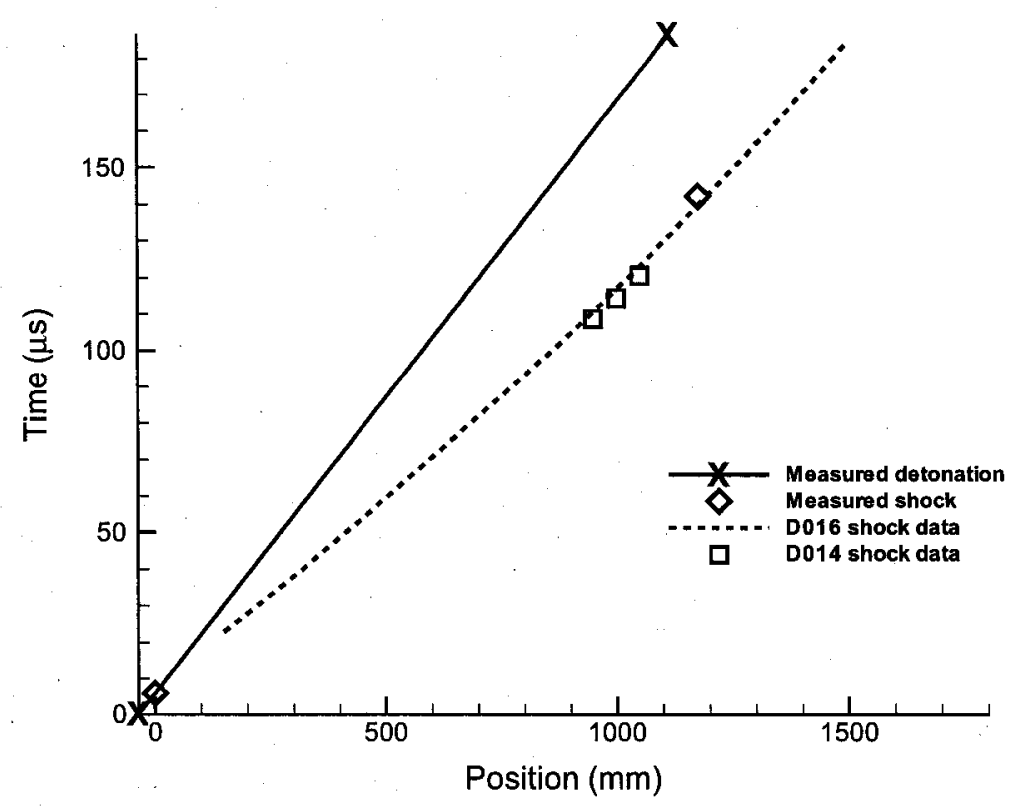

Figure 3-13: $x-t$ graph for an untamped driver with 20.4 atm initial fill pressure

\subsubsection{Shots Using Detasheet Explosives}

The explosive used in all driver shots described so far has been nitromethane. A series of driver experiments was done using a different explosive: detasheet, which has a detonation velocity of $7 \mathrm{~km} / \mathrm{s}$. In helium, an ideal piston traveling at this velocity drives a $9.4 \mathrm{~km} / \mathrm{s}$ shock ahead of it. Shots D019 used the $1.27 \mathrm{~cm}$ outer diameter, $0.9 \mathrm{~mm}$ wall thickness pump tubes used in shots D014 to D018. Shots D024 and D025 used $2.54 \mathrm{~cm}$ outer diameter pump tubes of different thicknesses. The details of each shot are given in table 3-3.

The probes used to measure the arrival times of the detonation wave in these experiments are ion probes instead of twisted pairs. One is inserted into the detasheet at each end of the tamper. These should not be affected by the expanding tube and provide more reliable detonation velocity measurements. However, for shots D019 
Table 3-3: Summary of driver experiments using detasheet explosives. Tampers for the $1.27 \mathrm{~cm}$ OD tubes were $4.45 \mathrm{~cm}$ OD, $1.91 \mathrm{~cm}$ ID steel tubes. Tampers for the $2.54 \mathrm{~cm}$ OD tubes were $7.62 \mathrm{~cm} \mathrm{OD}, 3.18 \mathrm{~cm}$ ID steel tubes.

\begin{tabular}{|c|c|c|c|c|c|}
\hline $\begin{array}{l}\text { Shot } \\
\text { No. }\end{array}$ & $\begin{array}{c}\text { Fill } \\
\text { Pressure } \\
\text { (atm) }\end{array}$ & $\begin{array}{l}\text { Detonation } \\
\text { Velocity } \\
(\mathrm{km} / \mathrm{s})\end{array}$ & $\begin{array}{l}\text { Shock } \\
\text { Velocity } \\
(\mathrm{km} / \mathrm{s})\end{array}$ & $\begin{array}{l}\text { Standoff } \\
(\mathrm{cm})\end{array}$ & Comments \\
\hline D019 & 15.6 & 7.47 & 8.73 & 14.13 & $\begin{array}{l}\text { Shock measured at } 94 \mathrm{~cm} \text { from } \\
\text { initial collapse point. Pump } \\
\text { tube and fill line not flushed } \\
\text { with helium before shot. Ap- } \\
\text { proximately } 18 \% \text { of the gas } \\
\text { mixture was air, which brought } \\
\text { down the shock velocity. }\end{array}$ \\
\hline D020 & 15.6 & 7.43 & 10.50 & 23.07 & $\begin{array}{l}\text { Repeat of shot D019, but the } \\
\text { fill line was flushed with helium } \\
\text { before the shot, not the tube. } \\
\text { The residual mass fraction of } \\
\text { air was small and the recorded } \\
\text { shock velocity is higher. }\end{array}$ \\
\hline D021 & 40.8 & $7.71^{\circ}$ & 8.33 & 17.63 & $\begin{array}{l}\text { Shock measured at } 128 \mathrm{~cm} \\
\text { from initial collapse point. }\end{array}$ \\
\hline D022 & 20.4 & 7.63 & 12.63 & 23.08 & $\begin{array}{l}\text { Shock measured at } 125 \mathrm{~cm} \\
\text { from initial collapse point. Un- } \\
\text { usually high precursor velocity. }\end{array}$ \\
\hline D023 & 40.8 & 7.23 & 7.18 & 19.55 & $\begin{array}{l}\text { No tamper was used. Ex- } \\
\text { plosive thickness was } 10 \mathrm{~mm} \text {. } \\
\text { Shock measured at } 127 \mathrm{~cm} \\
\text { from initial collapse point. }\end{array}$ \\
\hline D024 & 20.4 & 7.74 & 9.40 & 21.61 & $\begin{array}{l}\text { Pump tube was } 2.54 \mathrm{~cm} \text { OD, } \\
0.9 \mathrm{~cm} \text { wall thickness. Shock } \\
\text { measured at } 111 \mathrm{~cm} \text { from ini- } \\
\text { tial collapse point. }\end{array}$ \\
\hline D025 & 20.4 & 7.70 & 9.43 & 17.46 & $\begin{array}{l}\text { Repeat of shot D024, but with } \\
1.65 \mathrm{~cm} \text { wall thickness. }\end{array}$ \\
\hline
\end{tabular}




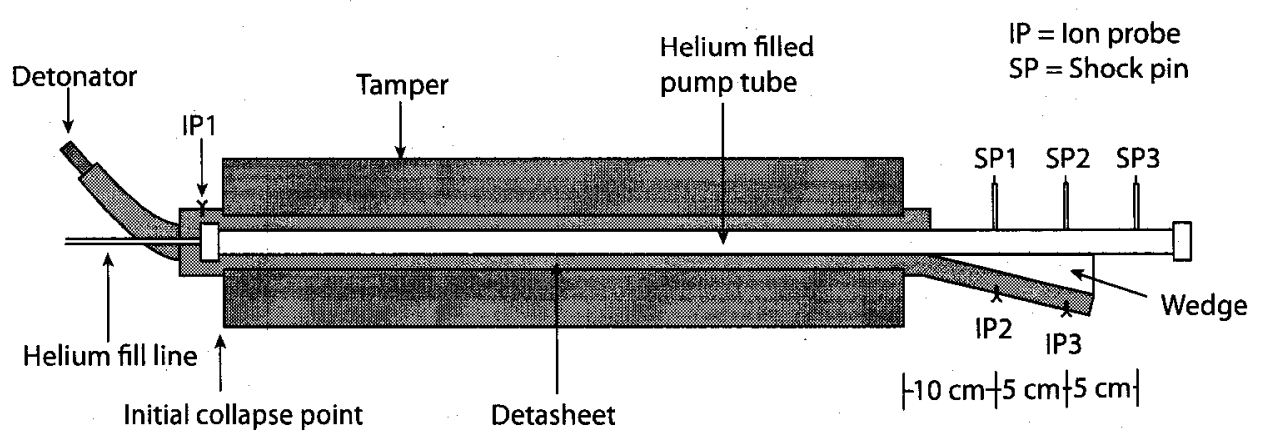

Figure 3-14: Experimental setup for driver shots using detasheet explosive

and D020, we still saw high detonation velocities. For the remaining tampered shots (D021 to D025, excluding D023), two ion probes were used at the exit of the tamper. These were moved away from the tamper and shielded from the pump tube with a wooden wedge. See figure 3-14 for a sketch of the experimental setup of these shots. With these precautions, the ion probes should provide the true time of arrival of the detonation at the end of the charge and eliminate any pre-shorting problems from pump tube swelling and bursting. We nevertheless saw high detonation velocities for all tamped shots. It is concluded that the precompression effect is present in tamped drivers and as a result the detonation velocity of the explosive is increased.

The general trend for driver performance $v s$ initial fill pressure observed in previous driver experiments is maintained in this series. Once again the performance of the untamped shot done in this series (D023) is quite close to that of the equivalent tamped shot (D021), although a thicker layer of explosives is required to collapse the pump tube. Shots D024 and D025 demonstrate the possibility of driving a precursor shock even for pump tube diameters as large as $2.54 \mathrm{~cm}$. They also support previous findings that a thinner pump tube wall is preferable. Recovered pump tubes from these experiments are more severely damaged than for tamped driver shots 


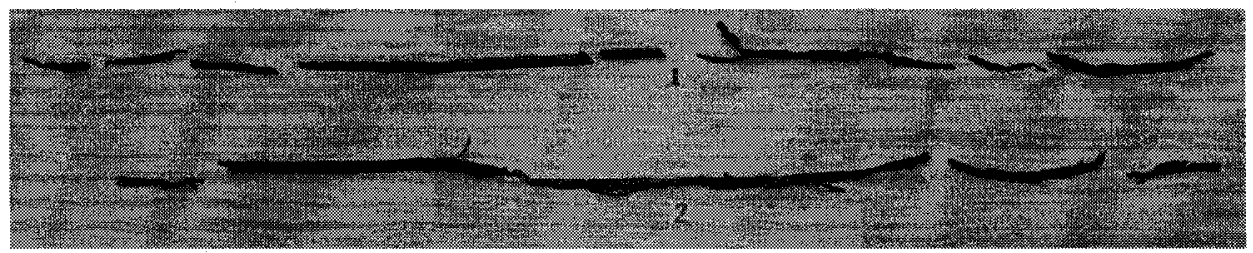

Figure 3-15: Photograph of the recovered pump tube fragments for shots D019 and D020

using nitromethane explosive. The recovered pump tube fragments for shots D019 and D020 are shown in figure 3-15.

\subsection{Conclusions}

The $0.64 \mathrm{~cm}$ driver shots clearly demonstrated that precursor shock velocity and standoff distance decrease as we increase the initial fill pressure of the driver. The $0.95 \mathrm{~cm}$ shots revealed that increasing the wall thickness of the pump tube is an ineffective strategy for increasing driver performance. In fact, shots D024 and D025 showed that a thinner pump tube wall thickness is preferable. For the $1.27 \mathrm{~cm}$ shots we see a decrease in driver performance as we increase the initial fill pressure, as with the $0.64 \mathrm{~cm}$ pump tube series. However, this decrease does not seem to be as pronounced as with the $0.64 \mathrm{~cm}$ pump tube series. A thinner layer of explosives is used to implode a larger tube in the $1.27 \mathrm{~cm}$ series than in the $0.64 \mathrm{~cm}$ series, so the role of the tamper in diminishing the amount of explosives needed has been proved, although its role in preventing pump tube bursting has not been verified. Finally, for tamped drivers, the compression of the explosive layer between the tamper and the expanding pump tube increases the detonation velocity of the explosive. The increase in velocity observed is greater than theory predicts for nitromethane explosive. This effect is not observed in untamped shots. 


\section{CHAPTER 4 \\ Theoretical Internal Ballistics}

We now turn our attention to the implosion-driven launcher. As mentioned in section 1.2.2, the launcher consists of a linear explosive driver followed by a chamber and a launch tube, inside of which is a projectile. Refer back to figure 1-4 for a schematic of launcher operation.

Of interest are the trajectory and velocity of the projectile, as well as the pressures applied to it. Ideally we would like to generate $x-t, x-v$, and $p-x$ diagrams, where $x$ is the position of the projectile down the launch tube, $t$ is time, $v$ is the velocity of the projectile, and $p$ is the pressure applied on the projectile.

If we consider that the virtual piston behaves as an ideal piston, that the projectile is rigid, and that the launch tube is evacuated, the launcher operation can be represented as a slug of gas between two boundaries: on one side we have a piston moving into the gas, and on the other we have a free mass which accelerates in response to the pressures applied to it. Solving for the internal ballistics of the launcher implies studying the response of the gas to both of these boundary conditions. We can treat this problem in one dimension, as we did when studying the cross-section of the pump tube in the expanding tube model in Chapter 2. Note that this means that all axial effects, such as expansion of the pump tube, chamber, and launch tube will not be considered here.

Two different codes were used to compute the internal ballistics of the launcher and estimate its performance: a method of characteristics code and an Eulerian CFD code. 


\subsection{Method of Characteristics}

A method of characteristics code was developed to solve the flow conditions of the driver gas. This method is similar to the acoustics method used in the expanding tube model discussed in section 2.1.4. Pressure waves are sent through the gas in response to disturbances it experiences. These waves, which propagate along lines called "characteristics," can be thought of as carriers of information through the gas. The difference here is that the flow cannot be considered to be nearly incompressible, so the speed of sound and the density of the gas will change. This means that these characteristics will not be straight lines. Different characteristics will have different slopes, meaning that we are not only concerned with interactions of the characteristics with the boundaries, but also with each other. At each interaction point, we can solve for the flow conditions. The result is a discretized solution of the flow field. Adding more characteristics to the problem increases the number of interactions and the accuracy of the method, but also increases the required number of computations and requires more time to solve.

A complete and detailed treatment of the method of characteristics is given in [13] and will not be reproduced here. Instead, the basic algorithm for the code and results will be given.

First we initialize the boundary conditions and characteristics, then allow them to propagate through the flow and interact with each other, solving for the flow conditions at each interaction until the end time or maximum number of interactions specified is reached. These are the steps followed by the code:

1. Based on known propagation velocities of the characteristics, check which ones will interact with each other. To save on computation time, the order of the 
characteristics is kept in memory so we can check for interactions between adjacent characteristics first.

2. Once a possible interaction is found, we find the interaction point and solve for the flow at that point. This is an iterative procedure, which accounts for non-isentropic flow. We also use an average characteristic slope between the prior points and the new point for added accuracy.

3. Step 2 is repeated for all possible interactions and the earliest one is retained. The data is then updated and logged in a matrix which stores all flow variables for each interaction point.

Shocks are treated as special characteristics in the sense that we require more information when solving for an interaction involving a shock. The Mach number of the shock as well as pre-shock and post-shock values of flow speed and sound speed need to be iterated for and retained for future interactions. We also need to account for the rise in entropy across the shock. Otherwise, shocks are treated as other characteristics and the steps described above are applied to them.

Once the computation has finished, we can plot the $x-t$ diagram. A sample diagram is shown in figure 4-2 for the following test case: a $0.5 \mathrm{~cm}$ diameter tube, initially filled helium at $50 \mathrm{~atm}$ and $300 \mathrm{~K}$, is imploded at a velocity of $6 \mathrm{~km} / \mathrm{s}$ and accelerates a lexan projectile weighing $0.1 \mathrm{~g}$ located $50 \mathrm{~cm}$ from the start point of the implosion. In this test case, the piston continues to travel down the tube until the end of the simulation. This means that we are imploding the launch tube as well. See figure $4-1$ for a sketch of this launcher.

The results of the simulation are shown in figure 4-2. Figure 4-2(a) displays all the characteristics. What is happening here at early times is clear: the piston is moving into the gas and generating a shock wave. The flow before the shock wave is 


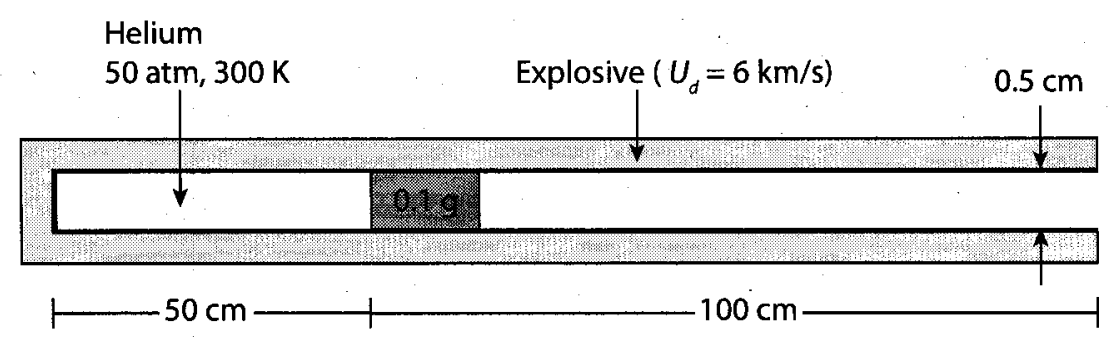

Figure 4-1: Diagram of the test case

undisturbed, indicating that the shock wave is propagating supersonically relative to it. Characteristics behind the shock wave travel between the piston and the shock, indicating that the flow is subsonic in this region.

Once the shock reflects off the projectile, it is difficult to see what is happening, therefore a plot of the boundaries with only the shock wave present is preferable from this moment on, as shown in figure 4-2(b). We can see here that the shock reverberates between the piston and the projectile a number of times. These reverberations raise the pressure applied to the projectile, and the result is an increase in its velocity.

The projectile velocity is updated every time a characteristic interacts with it. The projectile trajectory will be better resolved if there are more characteristics updating the pressure behind it. Referring back to figure 4-2(a), we see that as the projectile accelerates away, the characteristic mesh is getting more and more spread out. In other words, there are fewer characteristics per unit length of the domain, leading to less frequent interactions with the projectile. This is a source of error for the code, but since we typically are interested in launch tube lengths of about one meter, this error can be kept small.

The advantage of this code is that it uses a graphical method for solving the flow and is therefore relatively easy to understand. However, it is not a particularly robust 


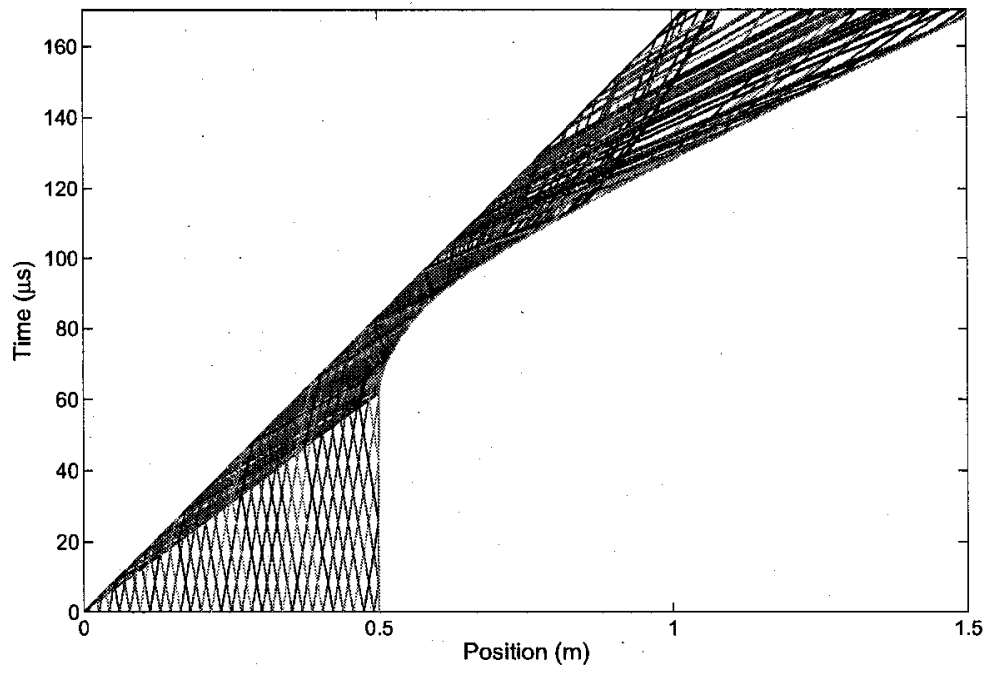

(a) All characteristics displayed

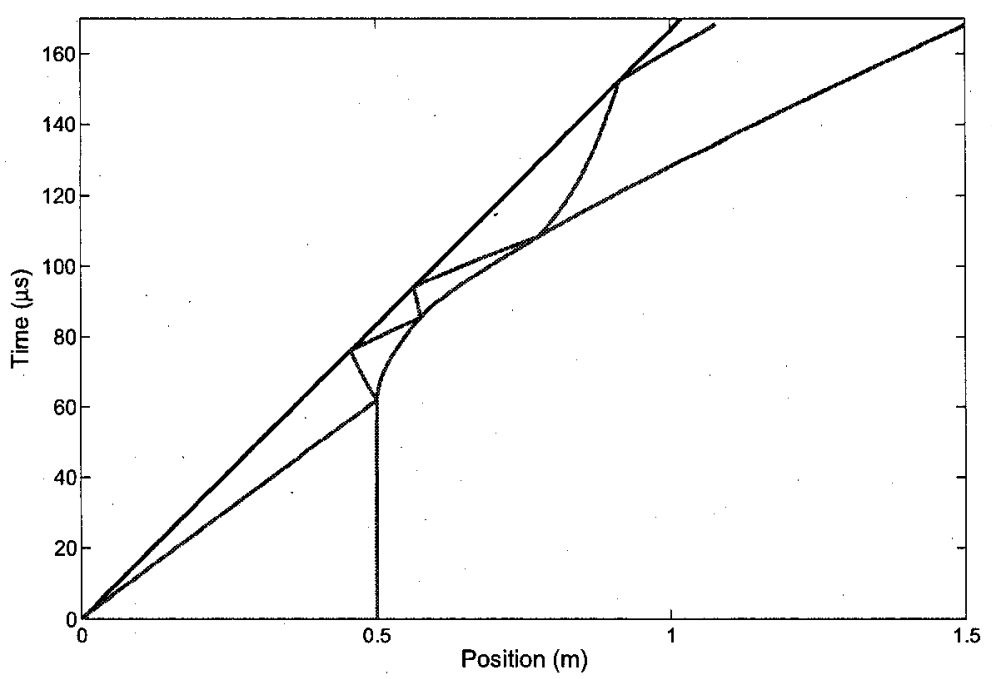

(b) Boundaries and shock only

Figure 4-2: Sample $x-t$ graph for the method of characteristics code 
code, especially when using a large number of characteristics. The code encounters problems solving for the next interaction when three or more waves meet at the same point, or when adjacent interactions occur at nearly the same time. In these cases, it may invert the order of the characteristics involved, or "forget" one. The effects of this type of error propagate and typically send the code into an infinite loop looking for the next interaction. Finally, to the point at which it was developed, this code cannot take area change into consideration, although the method of characteristics itself allows for changes in area. Adding chambrage is a way of increasing launcher performance, as explained in section 1.2.1. It is therefore important to be able to take area changes into account when simulating launcher operation.

\subsection{1-D Euler Code}

The second code used to simulate the launcher operation is a CFD code which solves the Euler equations for a quasi-one-dimensional finite volume of gas subjected to different boundary conditions [12]. It uses the SLIC (Slope Limiter Centered) scheme, which is described in [15], and can account for smooth changes in area; incorporating the effects of chambrage is therefore possible with this code.

The code solves for the flow conditions as time progresses based on the initial conditions and boundary conditions entered. The solution matrix is saved and kept in memory. It is then possible to obtain schlieren $x-t$ graphs, where density changes are represented on a monochromatic scale. See figure 4-3 for an example of this type of graph. The case simulated here is the same as that used for figure $4-2$. We can see the shock as the sharp dark edge on the schlieren graph reverberating as we saw in section 4.1.

When incorporating the area change section, we must make sure that the transition between the chamber area and the launch tube area is smooth and gradual; 


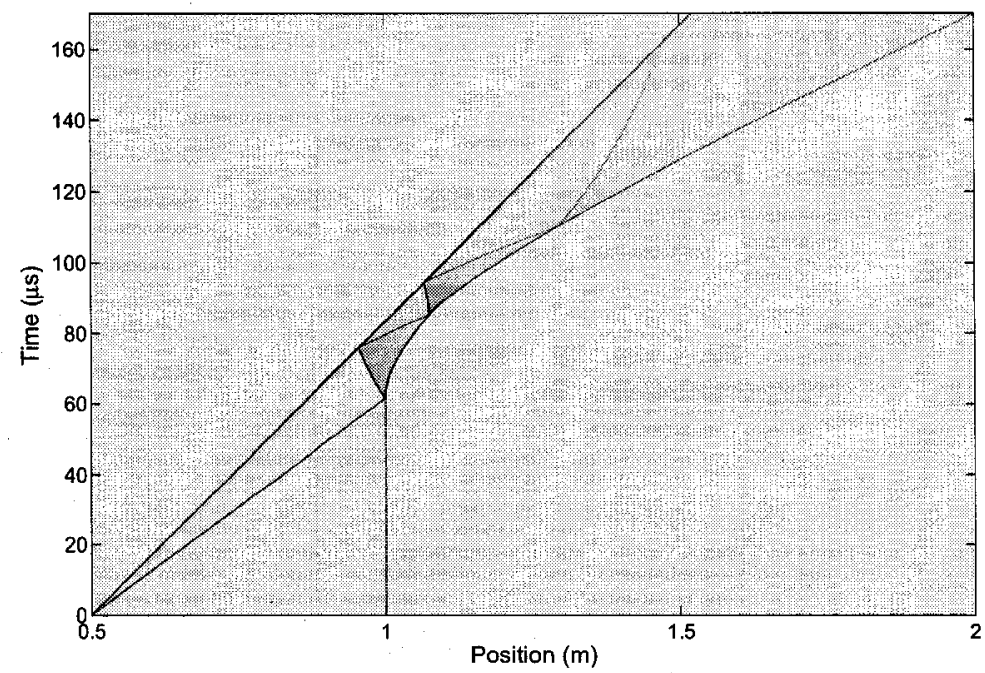

Figure 4-3: Sample computational $x-t$ schlieren from the Euler code

sharp changes in area should be avoided. In all cases, a convergence test must be done to ensure that the number of cells used is high enough to properly resolve the area change section.

\subsection{Validation of the Numerical Codes}

\subsubsection{Comparison of Both Codes With Each Other}

As a first check, results of both codes for a few test cases were compared with each other. One such test case was presented in sections 4.1 and 4.2 . Figure 4-4 shows the superimposed $x-t$ and $x-v$ graphs from both codes for this test case.

Both the $x-t$ (figure 4-4(a)) and $x-v$ (figure 4-4(b)) histories given by each code are virtually the same, and the final projectile velocities obtained are extremely close (within about $0.3 \mathrm{~km} / \mathrm{s}$ ).

The reason the method of characteristics code shows a slightly different shock wave trajectory at later times and gives a slightly higher projectile velocity is a consequence of the characteristic mesh getting more and more spread out, as discussed 


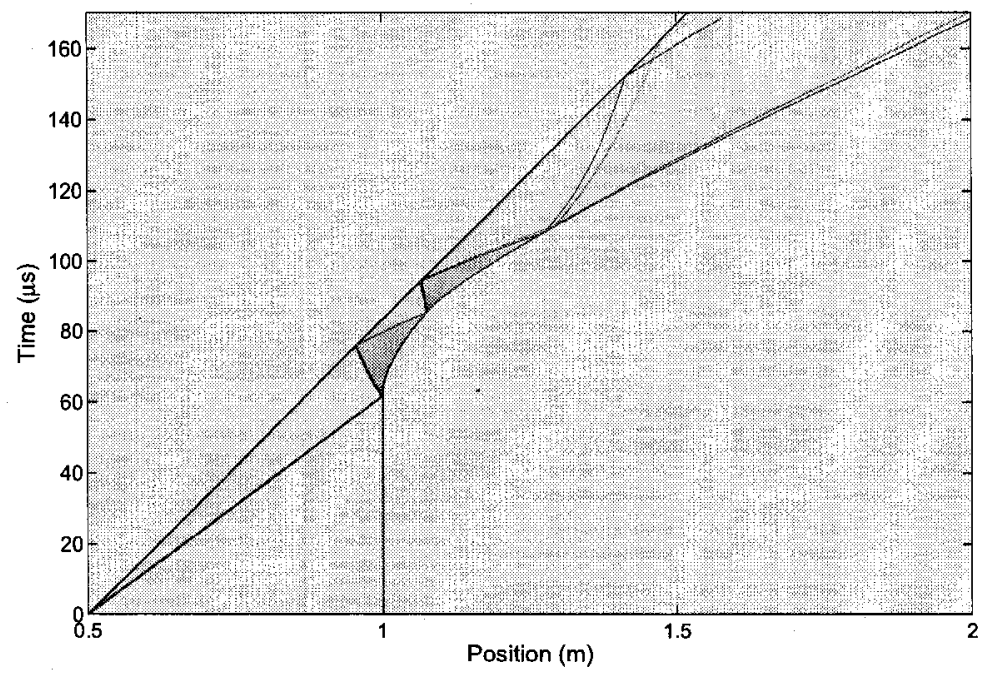

(a) $x-t$ history comparison

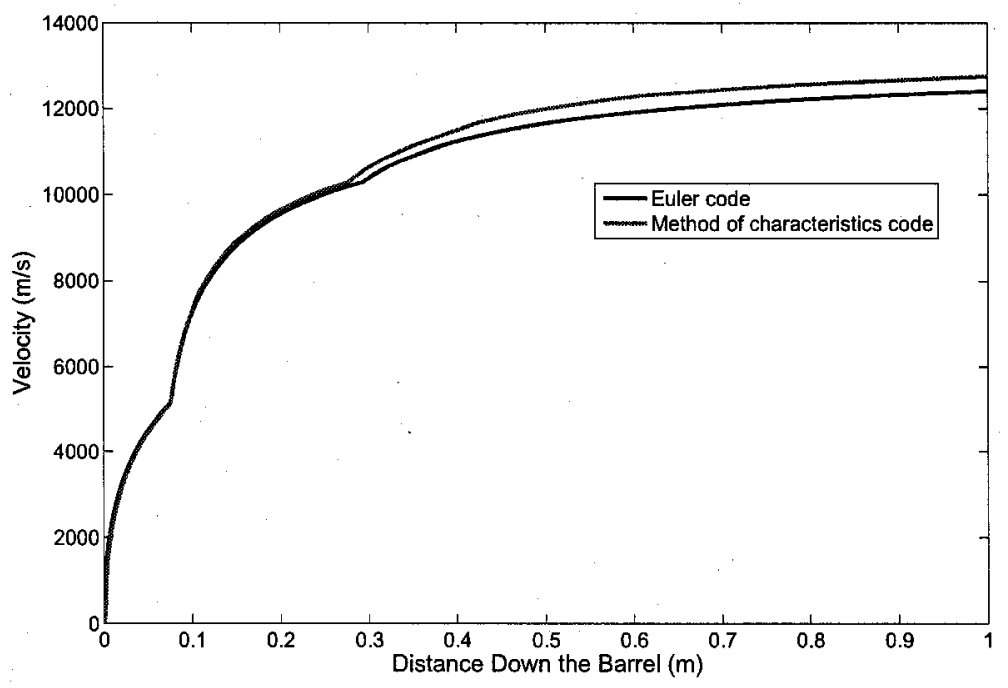

(b) $x-v$ history comparison

Figure 4-4: Comparison of results obtained from both codes for a sample test case 


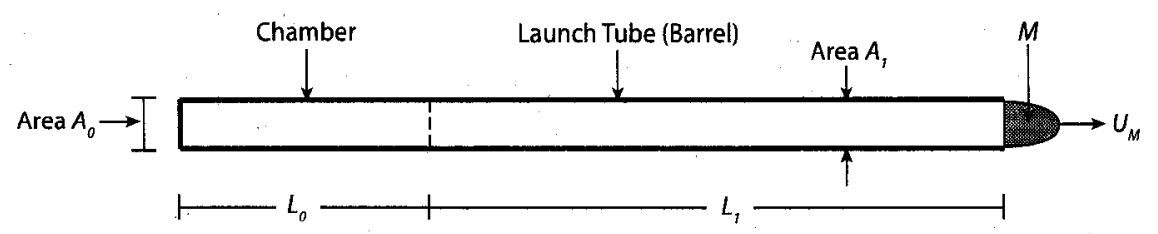

Figure 4-5: The unchambered, finite chamber length, PPIG launcher

in section 4.1. Now that we have established that both codes agree with each other, we need to confirm that they both agree with theory.

\subsubsection{Comparison of Both Codes With Theory}

Graphical data on launcher performance is presented in [14] for various launcher configurations. This data was used as a benchmark for testing both the Euler code and the method of characteristics code.

Both codes require a finite volume of gas to work with and the method of characteristics code cannot account for area changes. The data given in [14] for a finite chamber length, preburned propellant ideal gas (PPIG) launcher without chambrage configuration therefore lent itself as an ideal initial test case. This type of launcher is basically a length of tube of uniform cross section sealed at one end, as shown in figure $4-5$. The projectile is initially some distance away from the sealed end. The propellant may either be a pre-burned reactive propellant or an inert pressurized gas, which is treated as an ideal gas.

The data presented in [14] shows dimensionless projectile velocity vs dimensionless projectile travel distance for a range of $G / M$ ratios. The projectile velocity at the exit of the launch tube $(V)$ is normalized by the initial sound speed of the gas in the chamber $\left(c_{0}\right)$, and the distance traveled by the projectile (the launch tube length, $\left.L_{1}\right)$, is normalized using the mass of the projectile $(M)$, the initial sound speed and pressure $\left(p_{0}\right)$ of the gas in the chamber, and the area of the launch 
tube $\left(A_{1}\right)$. The results from both the method of characteristics code and the Euler code were nondimensionalized accordingly and plotted alongside the data from [14]. The resulting graph is shown in figure 4-7. Results from the codes for this case are found to match perfectly with those in the literature.

To test the area change capability of the Euler code, chambrage was added to the finite chamber length PPIG launcher. The results from the Euler code were nondimensionalized and compared to the data found in [14] as in the previous case. The resulting graph in shown in figure 4-7. Once again the results obtained through the code matches very well those found in the literature.

We are now certain that these codes are reliable tools which can be used to simulate the ideal ballistics of launcher experiments. Since the Euler code is easier to use and more versatile, it was chosen as the default tool for this task.

\subsection{Numerical Modeling of the Launcher}

The launchers to be used in experiments were simulated using the Euler code described above. All of these were of the type shown in figure 1-4 in section 1.2.2. The exact details on the design of the launcher are not necessary at this point. We only require the dimensions of the pump tube, the chamber, and the launch tube, as well as the mass of the projectile and the initial conditions of the driver gas used.

The size of pump tubes used in experiments was determined based on the driver experiments of Chapter 3. The $1.27 \mathrm{~cm}$ outer diameter stainless steel 304 tubes demonstrated good performance. Of the tubes experimented with, they are the largest for which it is possible to use the facilities at McGill University. A larger tube allows a larger amount of driver gas per unit length. This increases the $G / M$ ratio, which is closely linked to launcher performance (see section 1.2.1). The length of the pump tubes used in launcher experiments is $L_{p}=120 \mathrm{~cm}$. We would like 


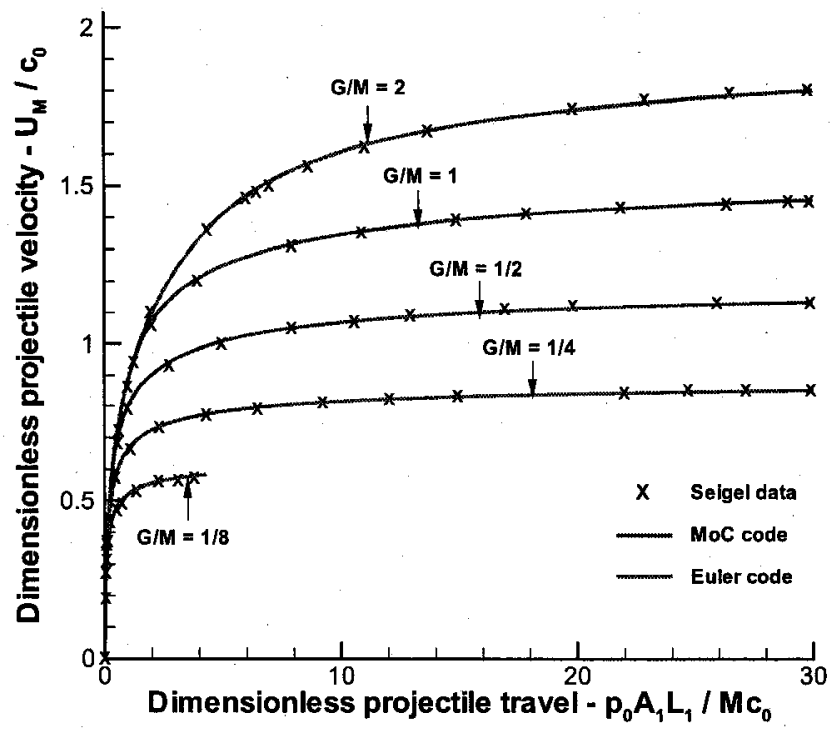

Figure 4-6: Performance of an unchambered, finite chamber length, PPIG launcher

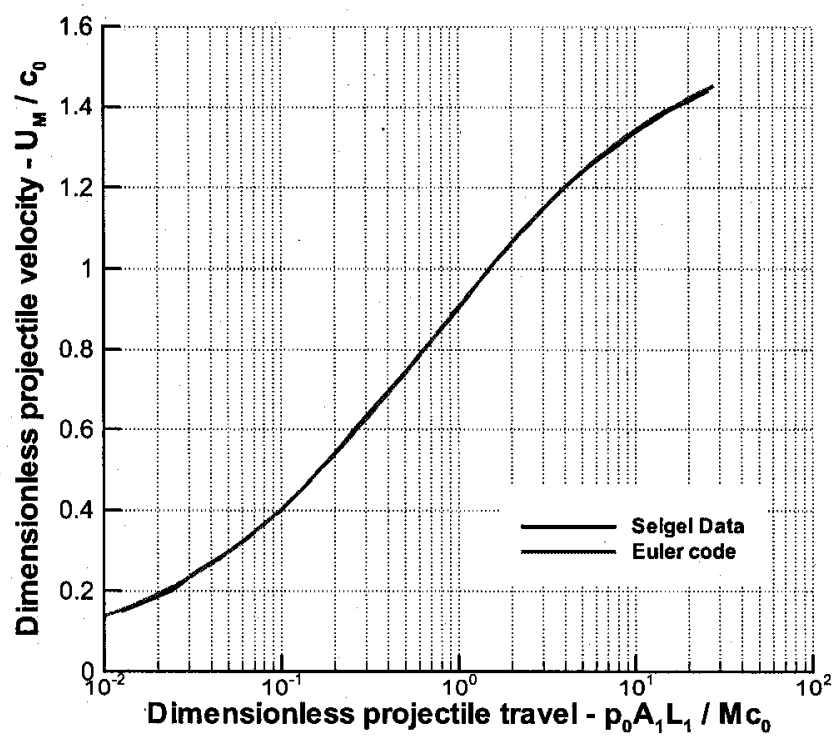

Figure 4-7: Performance of a finite chamber length, chambered, PPIG launcher $\left(D_{0} / D_{1}=1.778, \gamma=1.4, G / M=1\right)$ 
the initial fill pressure to be as high as possible without damaging the launcher and projectile. Based on the driver experiments of Chapter 3, an initial fill pressure of $20 \mathrm{~atm}$ yields good driver performance. This pressure should be low enough so that the projectile integrity is not compromised during launch. A minimum initial helium fill pressure of 20 atm was therefore selected. The projectile mass was chosen so that the $G / M$ ratio be at least unity at the lowest fill pressure used (20 atm). This translates to a projectile mass of about $0.3 \mathrm{~g}$. The launch tube was made about $0.9 \mathrm{~m}$ long because of experimental size restrictions. Projectile velocity estimates were therefore taken for a launch tube length of $0.9 \mathrm{~m}$. The launch tube inner diameter is $0.48 \mathrm{~cm}$. This provides some chambrage to the launcher $\left(D_{0} / D_{1} \approx 2.3\right)$ and permits a reasonable length-to-diameter ratio for the projectile $(\approx 1.3)$. A projectile having a low length-to-diameter ratio (below 1) may tumble in the launch tube as it is accelerating. The area change section between the chamber and the launch tube measures $L_{A}=0.75 \mathrm{~cm}$.

The length $L_{0}$ of the chamber is not as obvious to determine. This length represents the portion of the pump tube which is not imploded by the detonation (see figure 4-8). Having a shorter chamber means we compress the driver gas further, leading to higher pressures. Thus, we can define a compression ratio $(C R)$ as the initial volume over the final volume of driver gas in the pump tube, assuming no gas exits the pump tube before the collapse process is complete. Since the pump tube cross sectional area does not change, the compression ratio is equivalent to the initial length of the pump tube over the chamber length:

$$
C R=L_{p} / L_{0}
$$




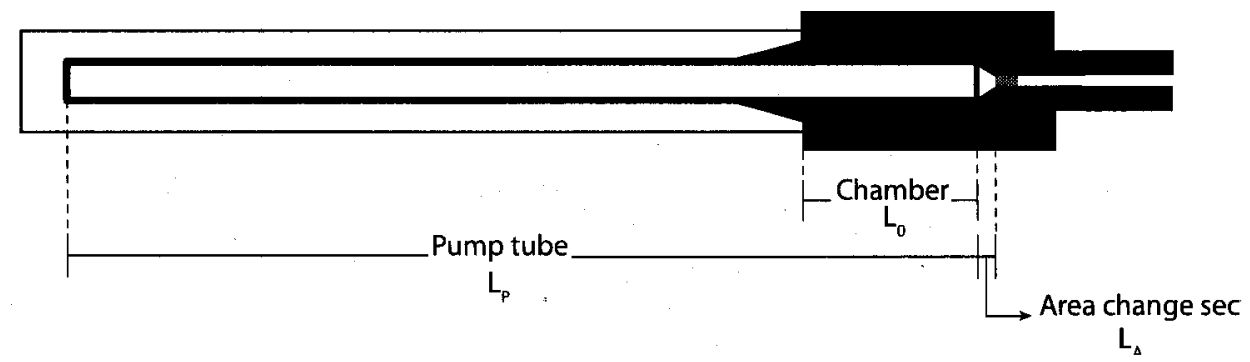

Figure 4-8: Definitions of launcher dimensions

A higher compression ratio leads to a higher projectile acceleration, but also a higher risk of damaging the projectile and the launcher. In particular, the pressure behind the reflected shock (the precursor reflected off the area change section or projectile, as discussed in section 1.2.2) is several times greater than behind the precursor shock. It is preferable then that the pump tube outside the chamber is not exposed to the reflected shock pressure. This means that the breech cone should be collapsed by the detonation before the shock can exit the chamber.

A series of simulations was done to determine the effects of chamber length. The dimensions given above were used for the launcher. The nominal chamber length was approximately calculated based on data from shot D016 so that the reflected shock would meet the detonation wave at the back end of the chamber. This length was determined to be about $13.5 \mathrm{~cm}$, and corresponds to a compression ratio of 8.9 . Euler code calculations were run for chamber lengths up to $6 \mathrm{~cm}$ longer and $6 \mathrm{~cm}$ shorter. This corresponds to compression ratios in the range of roughly 6 to 16 .

Figure 4-9 compares the computational $x-t$ schlieren diagrams obtained from the Euler code for two different compression ratios. Notice that as we increase the compression ratio (i.e. decrease the chamber length), the shock reflections become more numerous, thus raising the pressure of the driver gas. The timing of the interactions also changes. For example, the reflected shock encounters a rarefaction wave 


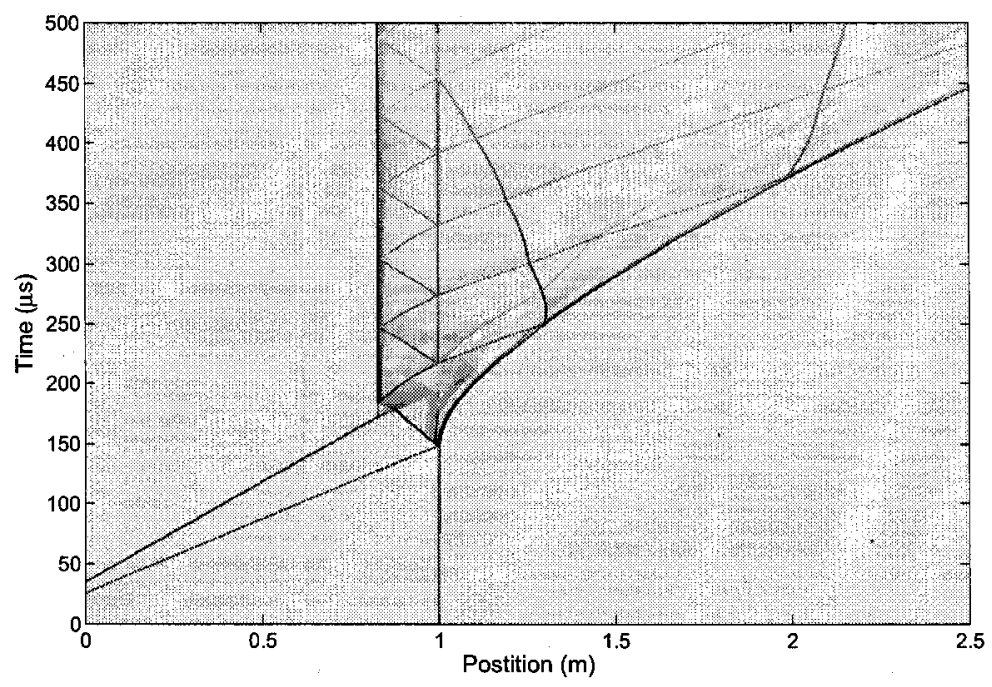

(a) $C R=7.3$

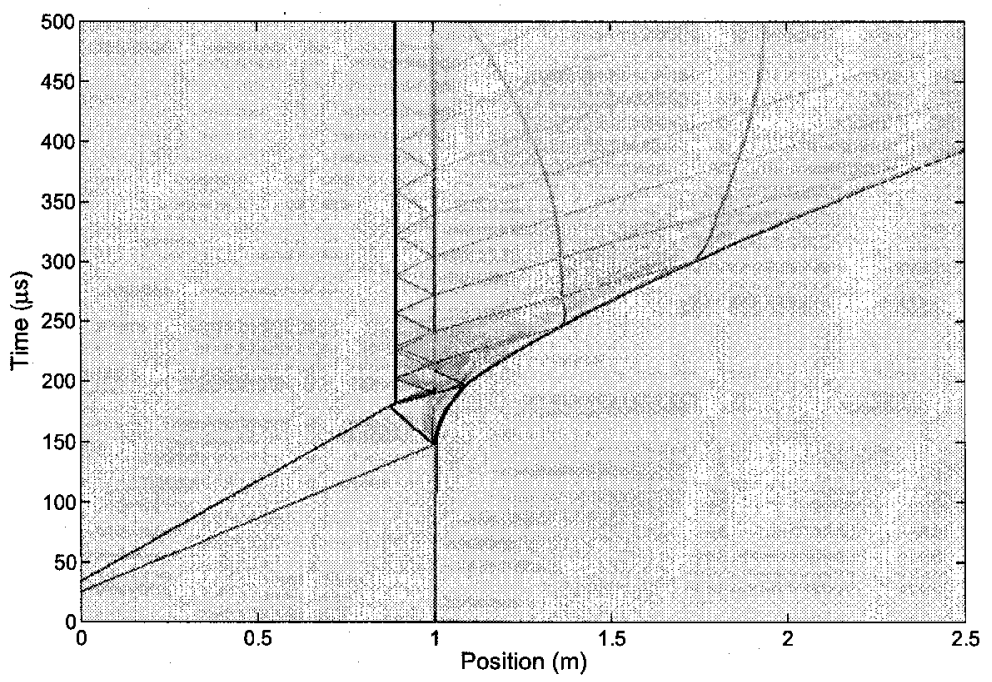

(b) $C R=11.5$

Figure 4-9: Computational $x-t$ schlieren from the Euler code for different compression ratios. Initial fill pressure is $30 \mathrm{~atm}$. 
before reflecting off a stationary piston in the $C R=7.3$ case. In the $C R=11.5$ case, the shock reflects directly off the traveling piston. The result is a much stronger re-reflected shock in the latter case. The limiting case is for $C R=8.9$, where the shock encounters the piston as it stop moving; it therefore hits a stationary piston, but without crossing a rarefaction first.

We can also obtain the velocity profile for the projectile as a function of distance traveled down the launch tube. Figure 4-10 shows such velocity profiles for various compression ratios. As shocks reflect off the projectile, they cause a sudden increase in pressure, which results in increased acceleration for a short time. This is what causes the "bumps" in the velocity profiles. The first shock reflection is the same in all cases, which explains why all the profiles are identical up to about $10 \mathrm{~cm}$ down the launch tube. The timing and strength of subsequent reflections depend on the chamber length. Notice that for the two highest compression ratios shown here, the second shock reflection appears identical. This is because the reflected shock reflects directly off the traveling piston in both cases, as explained above for the $C R=11.5$ case and shown in figure 4-9(b). The second shock hitting the projectile is therefore the same.

From these velocity profiles, we can sample the projectile velocity for a $0.9 \mathrm{~m}$ long launch tube. Velocities were obtained for compression ratios in the range of 6 to 16 for initial fill pressures from $20 \mathrm{~atm}$ to $50 \mathrm{~atm}$. The results are shown in graphical form in figure 4-11. The Euler code predicts that the projectile velocity increases with compression ratio and with pressure. Note that there is a kink in the curves at a compression ratio of about 10 . This is slightly above the point where the reflected shock goes from hitting a stationary piston after going through a rarefaction wave to hitting a traveling piston, as discussed above and depicted in figure 4-9. For 


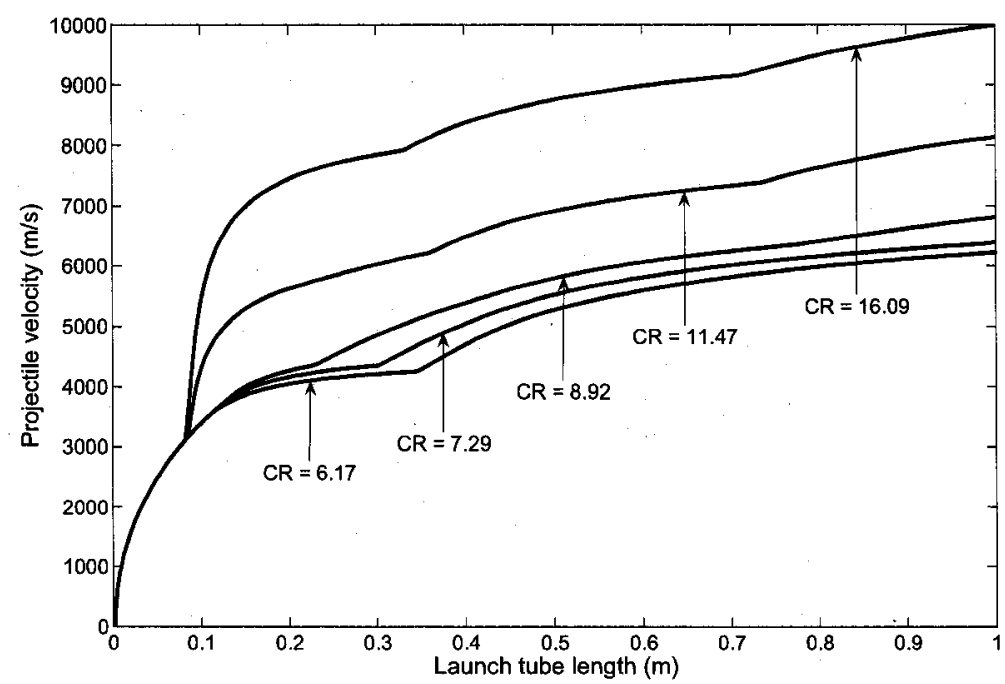

Figure 4-10: Projectile velocity profiles as a function of distance traveled down the launch tube for various compression ratios. Initial fill pressure is $30 \mathrm{~atm}$.

compression ratios above 10 , the shock has time to hit the projectile before the rarefaction generated by the stopped piston can attenuate it significantly.

It is important to remember that these velocity estimates are absolute upper bounds on the capabilities of the launcher. The Euler code assumes the implosion pinch acts as an ideal piston and it does not incorporate yielding, bursting, or ablation of the inner walls of the launcher from the extremely high pressure and temperature driver gas. In experiments, we do not expect the launcher performance to increase indefinitely with compression ratio and fill pressure. Instead, we expect there will be an optimum operating point beyond which the launcher and/or projectile will fail due to the extreme pressures reached.

We also expect to have difficulty properly sealing the chamber. In the above Euler code calculations, the piston remains in place once it reaches the chamber, forming a breech and preventing driver gas from exiting the chamber. In experiments, 


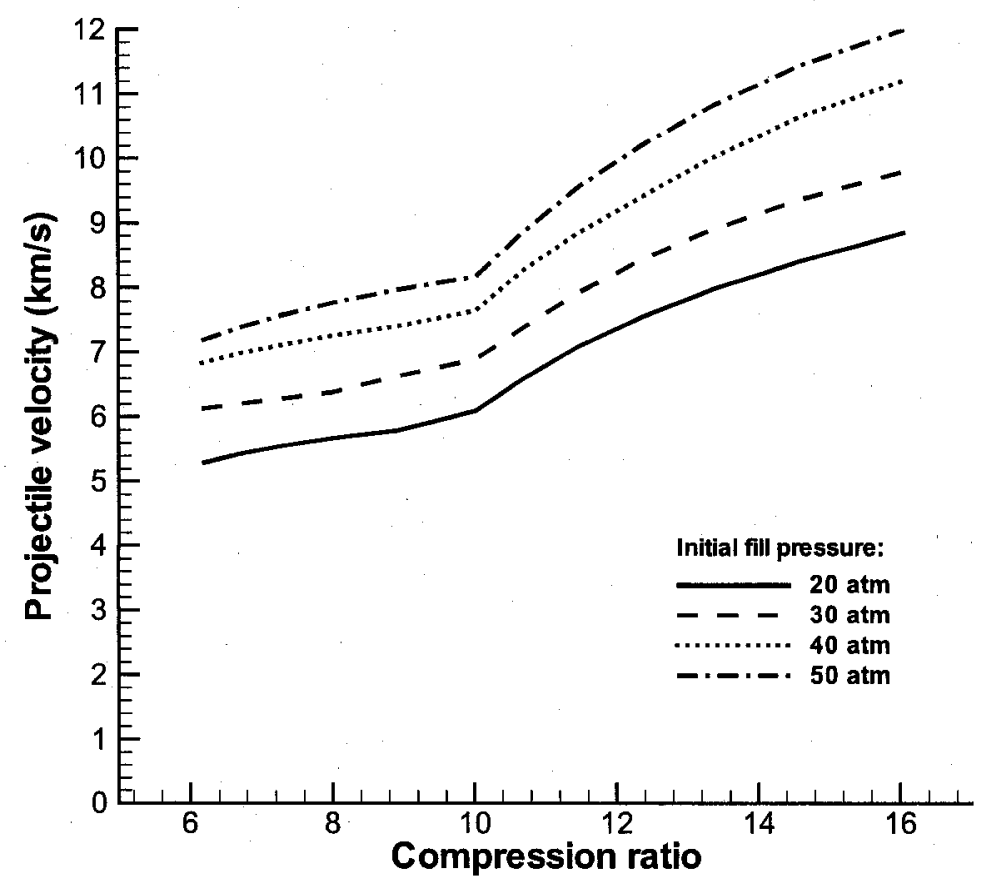

Figure 4-11: Projectile velocity as a function of compression ratio for various initial fill pressures. The piston seals the chamber once it reaches it. 
it is likely that the seal will be imperfect or absent. It is therefore relevant to estimate the effect of a venting chamber on the performance of the launcher.

Simulations were done where the piston, after reaching the chamber, moves away from the chamber at the detonation velocity of the explosive $(6 \mathrm{~km} / \mathrm{s})$ and allows the driver gas to expand out of the chamber. This simulates the destruction of the piston once it reaches the chamber. A piston retreating from the chamber at higher velocities does not influence the projectile trajectory significantly, as can be seen in figure 4-12. This is because the piston is close to the free expansion velocity of the gas. In figure 4-12(b), the piston exceeds the free expansion velocity of the gas, and we can see the leading edge of the free expansion in the $x-t$ graph. The space between the piston and the leading edge of the free expansion is under vacuum. Note that these are extreme cases. We do not expect the chamber to lose driver gas at the rates simulated here.

Again we see two distinct regimes in launcher operation which depend on the compression ratio, as we did in figure 4-9. For compression ratios under 10 , the projectile experiences only one shock reflection. The shock is then attenuated and disappears due to driver gas venting out of the chamber, as shown in figure 4-13(a). For compression ratios above 10 , the projectile experiences a second shock reflection. This is because the shock is allowed to reflect off the piston as it is still moving toward the chamber, as shown in figure $4-13(\mathrm{~b})$. The effect of the venting chamber is only felt after the shock has reflected off the projectile a second time. The second shock reflection imparts an extra increase in pressure and results in higher muzzle velocities, as shown in figure 4-13(c).

We can now plot the projectile muzzle velocity for compression ratios in the range of 6 to 16 and for initial fill pressures from 20 atm to 50 atm where the 


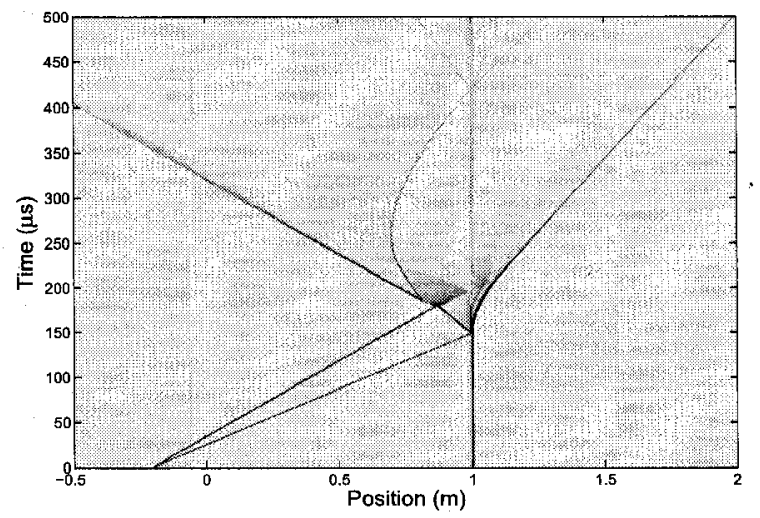

(a) Piston velocity of $6 \mathrm{~km} / \mathrm{s}$

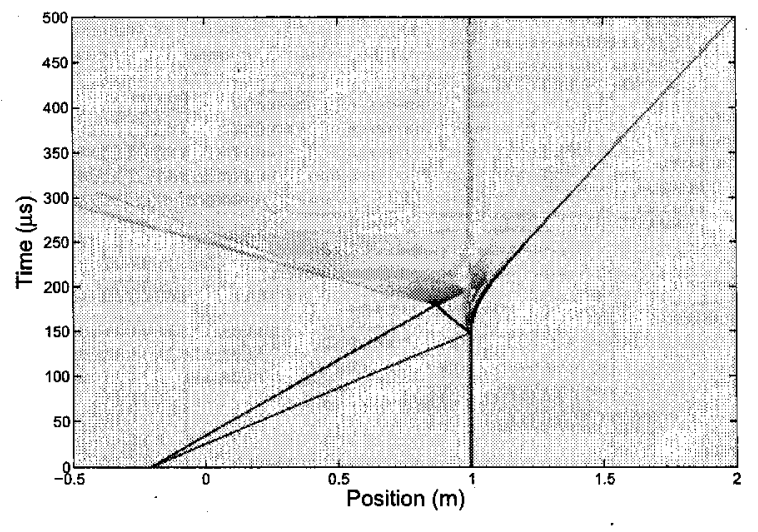

(b) Piston velocity of $12 \mathrm{~km} / \mathrm{s}$

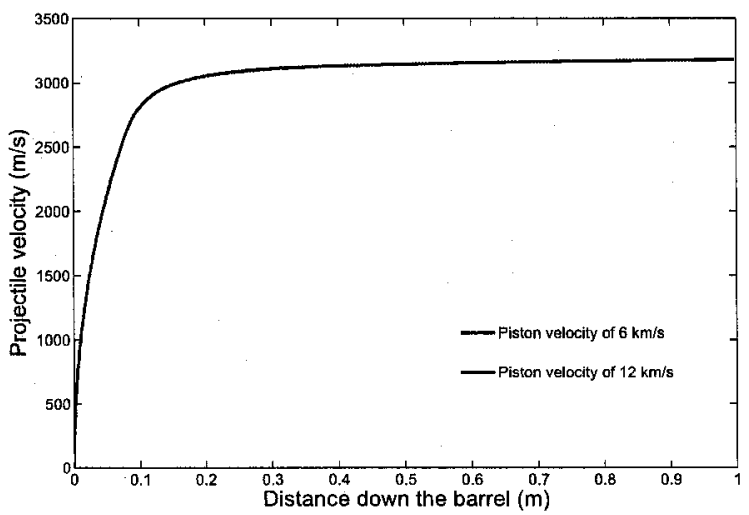

(c) $x-v$ history comparison

Figure 4-12: Computational $x-t$ and $x-v$ graphs from the Euler code for different retreating piston velocities. Initial fill pressure is $20 \mathrm{~atm}, C R=8.9$ 
piston does not seal the chamber and driver gas is allowed to vent out. As can be seen in the resulting graph (figure 4-14) the performance of the launcher does not increase significantly for compression ratios under 10 , then progressively increases for compression ratios above 10 .

Note that we said above that it is preferable that the pump tube outside the chamber is not exposed to the reflected shock pressure. This happens for compression ratios above 8.9. However, if the chamber is not sealed, we need a compression ratio above 10 to note a significant increase in driver performance. It is therefore worthwhile to perform launcher experiments to determine where the optimal launcher operation point is. 


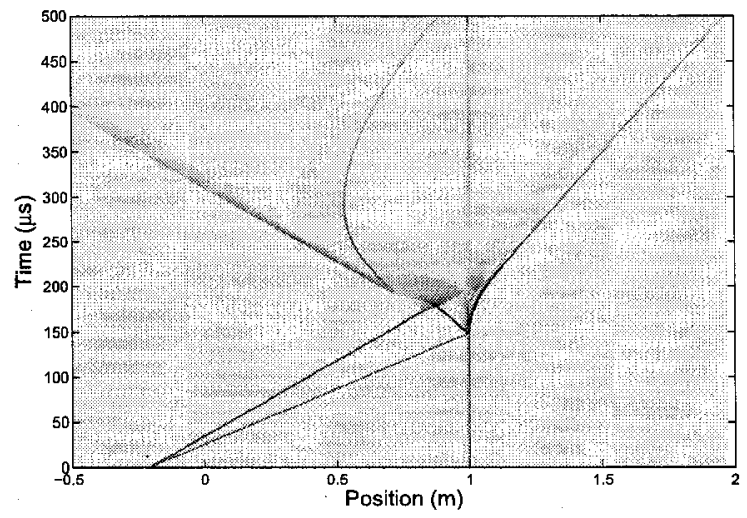

(a) $x-t$ schlieren for $C R=7.3$

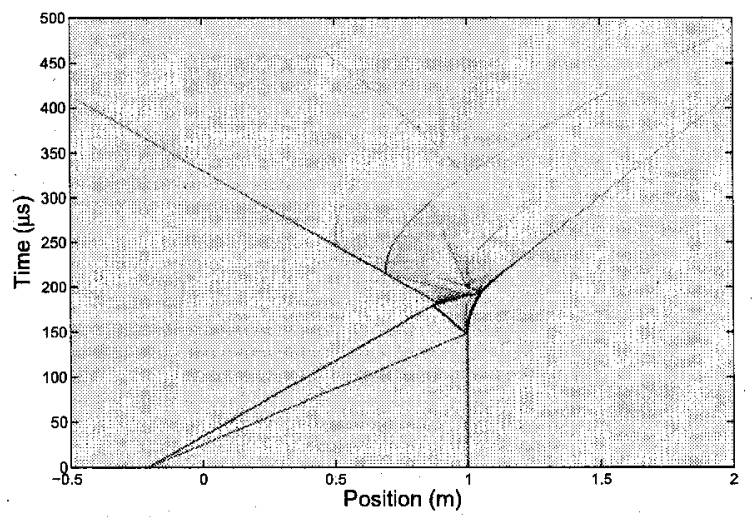

(b) $x-t$ schlieren for $C R=11.5$

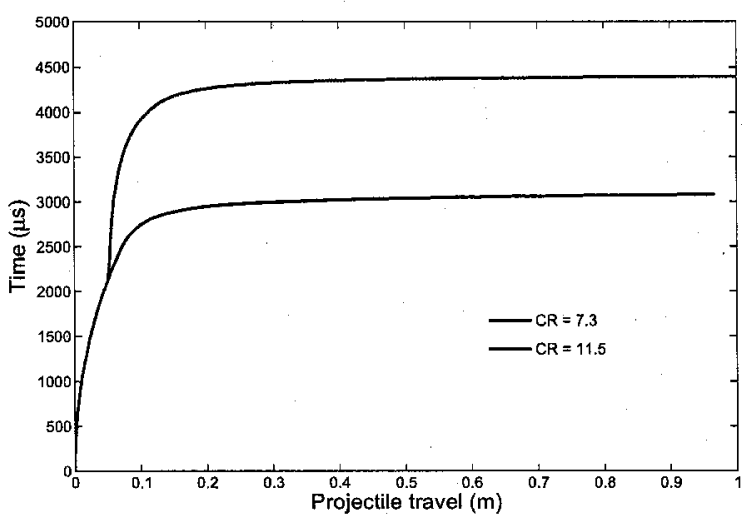

(c) $x-v$ history comparison

Figure 4-13: Computational $x-t$ and $x-v$ graphs from the Euler code for different compression ratios. Initial fill pressure is $20 \mathrm{~atm}$. 


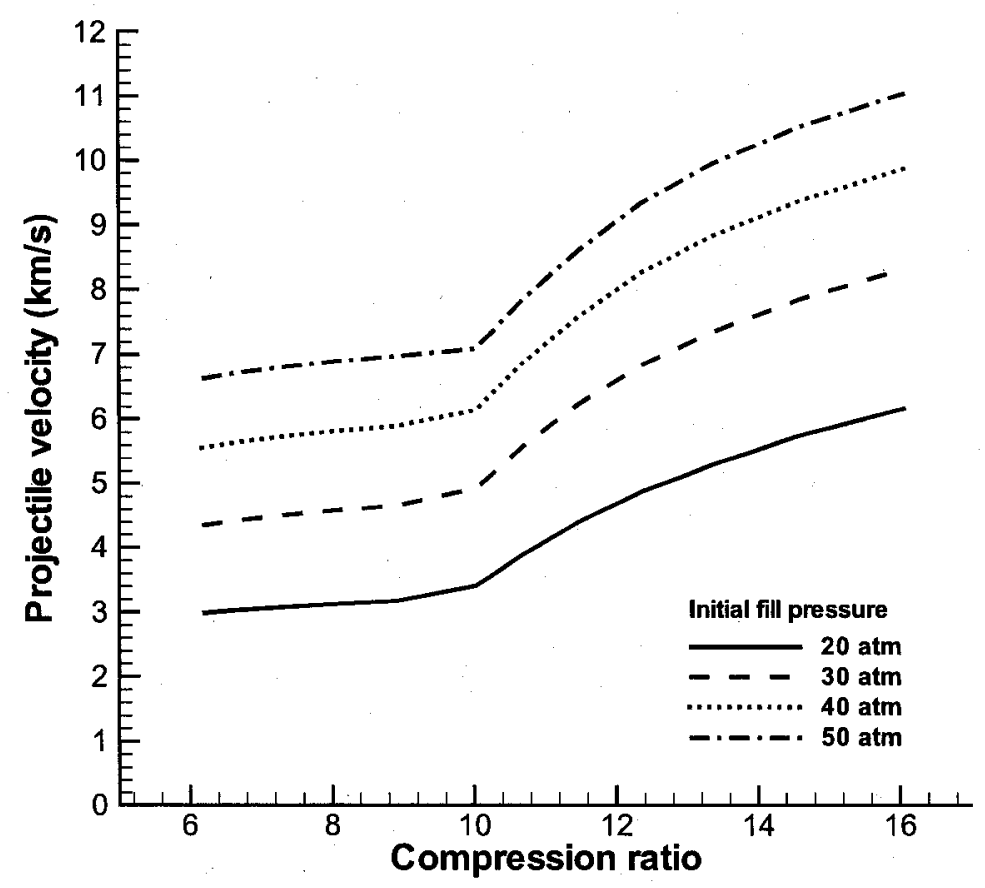

Figure 4-14: Projectile velocity as a function of compression ratio for various initial fill pressures. The piston reverses direction once it reaches the chamber to simulate its destruction. 


\section{CHAPTER 5 \\ Launcher Experiments}

Ideal numerical launcher simulations were done in the previous chapter using a quasi-one-dimensional Eulerian solver. These simulations predict an increase in launcher performance as we increase the compression ratio and initial driver gas fill pressure. However, the solver makes a number of ideal assumptions, so that the predicted performance of a given launcher is the maximum attainable experimental performance for that launcher. We also expect an optimum fill pressure and compression ratio beyond which increasing one or the other will not result in an equivalent increase in performance due to damage to the launcher and/or projectile.

\subsection{Launcher Design}

The initial launcher experiments were mostly aimed at gaining knowledge about how to design the launcher and measure projectile velocity. Much iteration was done on launcher design and diagnostics setup. For these early launcher experiments, it is most useful to concentrate on the acquired knowledge rather than on the individual shots, however the details can be found in tables $5-1$ and $5-2$.

\subsubsection{Launcher Chamber}

The linear explosive driver delivers the hot high-pressure driver gas it generates into the chamber of the launcher. The chamber then has to contain the driver gas and allow it to expand into the launch tube in order for it to propel the projectile. The pressure of the driver gas is transient during launch due to the shock reverberating between the virtual piston and the projectile. Chamber failure results in loss of driver gas and hence loss of performance. Therefore, the chamber walls should be 
Table 5-1: Summary of launcher experiments using $0.95 \mathrm{~cm}$ outer diameter, $1.65 \mathrm{~mm}$ wall thickness pump tubes

\begin{tabular}{|c|c|c|c|c|}
\hline $\begin{array}{l}\text { Shot } \\
\text { No. }\end{array}$ & $\begin{array}{l}\text { Fill } \\
\text { Pressure } \\
(\mathrm{atm})\end{array}$ & $\begin{array}{l}\text { Projectile } \\
\text { Velocity } \\
(\mathrm{km} / \mathrm{s}) \\
\end{array}$ & $\begin{array}{c}\text { Projectile } \\
\text { Mass } \\
(\mathrm{g})\end{array}$ & Comments \\
\hline L001 & 26 & - & 0.077 & $\begin{array}{l}\text { Pump tube length was } 60 \mathrm{~cm} \text {. The chamber was } \\
\text { a Swagelok VCO face seal. Projectile was made } \\
\text { of lexan. Projectile velocity measured with break } \\
\text { screens and twisted pairs within the launch tube. } \\
\text { Projectile was not recovered. }\end{array}$ \\
\hline $\mathrm{L} 002$ & 33 & - & 0.075 & $\begin{array}{l}\text { The chamber consisted of two flanges bolted to- } \\
\text { gether. The twisted pairs in the launch tube gave } \\
\text { signals out of sequence. Break screens were not used } \\
\text { since they are unreliable. Projectile was not recov- } \\
\text { ered. }\end{array}$ \\
\hline L003 & 34 & - & 0.077 & $\begin{array}{l}\text { Pump tube length increased to } 65 \mathrm{~cm} \text {. Projectile was } \\
\text { not recovered. }\end{array}$ \\
\hline L004 & 34 & $\overline{-}$ & 0.839 & $\begin{array}{l}\text { Twisted pairs in launch tube were not used since they } \\
\text { are recording a wave transmitted in the launch tube, } \\
\text { not the projectile. Used lasers to record projectile } \\
\text { speed. Changed projectile material from lexan to } \\
\text { aluminum to ensure projectile survival. Projectile } \\
\text { was recovered. Main purpose was to test lasers. }\end{array}$ \\
\hline $\mathrm{L} 005$ & 41 & 0.9 & 0.85 & $\begin{array}{l}\text { Projectile was recovered. Main purpose was to test } \\
\text { lasers. }\end{array}$ \\
\hline L006 & 41 & - & 0.84 & $\begin{array}{l}\text { Projectile was recovered. The chamber leaks driver } \\
\text { gas once the shock hits the diaphragm. Two lasers } \\
\text { are not enough: the beams are hard to hit, and if we } \\
\text { miss one, we obtain no velocity data. }\end{array}$ \\
\hline L007 & 34 & 1.56 & 0.83 & $\begin{array}{l}\text { The chamber was a thick tube welded to a nut, which } \\
\text { screwed onto a threaded rod. Two lasers were added. } \\
\text { Good velocity measurement for this shot. }\end{array}$ \\
\hline L008 & 34 & 1.58 & 0.538 & $\begin{array}{l}\text { Used a lighter projectile to gain velocity, but the } \\
\text { gain was minimal. Launch tube material changed } \\
\text { to chrome-moly steel. }\end{array}$ \\
\hline L009 & 34 & 2.7 & 0.535 & $\begin{array}{l}\text { First tamped launcher shot. Tamper was made of } \\
\text { two tubes; one slid into the other. Unreliable laser } \\
\text { data. The tamper made it hard to work with twisted } \\
\text { pairs; two of them shorted prior to the shot. }\end{array}$ \\
\hline L010 & 13 & 1.64 & 0.33 & $\begin{array}{l}\text { Chamber used a } 3 \text {-piece design with a nipple for a } \\
\text { better seal. } 5.08 \mathrm{~cm} \text { OD, } 2.54 \mathrm{~cm} \text { ID steel tube was } \\
\text { used as a tamper. It was lined on the inside with } \\
2.54 \mathrm{~cm} \text { OD, } 2.22 \mathrm{~cm} \text { ID PET tube. }\end{array}$ \\
\hline
\end{tabular}


Table 5-2: Summary of initial launcher experiments using tamped $1.27 \mathrm{~cm}$ outer diameter, $0.9 \mathrm{~mm}$ wall thickness pump tubes

\begin{tabular}{|c|c|c|c|c|}
\hline $\begin{array}{l}\text { Shot } \\
\text { No. }\end{array}$ & $\begin{array}{c}\text { Fill } \\
\text { Pressure } \\
\text { (atm) }\end{array}$ & $\begin{array}{c}\text { Projectile } \\
\text { Velocity } \\
(\mathrm{km} / \mathrm{s})\end{array}$ & $\begin{array}{c}\text { Projectile } \\
\text { Mass } \\
\text { (g) }\end{array}$ & Comments \\
\hline L011 & 20 & 3.8 & 0.33 & $\begin{array}{l}\text { Tamper was a } 5.08 \mathrm{~cm} \text { OD } 2.54 \mathrm{~cm} \text { ID steel tube. It } \\
\text { was lined on the inside with } 2.54 \mathrm{~cm} \text { OD, } 2.22 \mathrm{~cm} \text { ID } \\
\text { PET tube. Bow shock from the projectile measured } \\
\text { at about } 3.8 \mathrm{~km} / \mathrm{s} \text { using an array of shock pins. The } \\
\text { lasers gave no reliable signal. }\end{array}$ \\
\hline L012 & 15 & 2.53 & 0.47 & $\begin{array}{l}\text { Tamper was changed to a } 4.45 \mathrm{~cm} \text { OD } 1.91 \mathrm{~cm} \text { ID } \\
\text { steel tube. Bow shock from the projectile measured } \\
\text { at about } 2.6 \mathrm{~km} / \mathrm{s} \text {. An extra shock pin was added } \\
\text { the shock pin array. Projectile jammed inside the } \\
\text { launch tube upon insertion due to a deformation of } \\
\text { the launch tube caused by brazing it to the chamber. } \\
\text { An extra sleeve was added to prevent the launch tube } \\
\text { from bursting. }\end{array}$ \\
\hline L013 & 15 & $\begin{array}{l}3.1 \\
\end{array}$ & 0.45 & $\begin{array}{l}\text { Bow shock from the projectile measured at about } \\
3.1 \mathrm{~km} / \mathrm{s} \text {. A tapered bushing slid onto the pump } \\
\text { tube served as a makeshift breech cone. The cone } \\
\text { was collapsed, but was blown away from the chamber } \\
\text { with the pump tube. }\end{array}$ \\
\hline L014 & 20 & - & 0.16 & $\begin{array}{l}\text { Bow shock from the projectile measured at about } 4.0 \\
\text { to } 4.6 \mathrm{~km} / \mathrm{s} \text {. Used a magnesium projectile with a } \\
\text { lexan obturator of the same dimension as the pro- } \\
\text { jectile, with a Bridgman cup at one end; its mass } \\
\text { was } 0.10 \mathrm{~g} \text {. The projectile was placed closer to the } \\
\text { diaphragm than intended, but it could not be moved } \\
\text { back without being damaged. No projectile was re- } \\
\text { covered, and velocity readings were inconclusive. A } \\
\text { breech cone identical to that in shot L013 was used, } \\
\text { with similar results. }\end{array}$ \\
\hline
\end{tabular}


thick to contain the high-pressure driver gas. The chamber needs to seal the initial fill pressure on the pump tube side in order to prevent the projectile from accelerating due to this initial pressure, which is typically tens of atmospheres. This is done with a diaphragm and o-ring. There is an area change section included within the chamber where we transition from the pump tube diameter to the launch tube diameter. Finally, the chamber should be relatively simple and inexpensive to produce as it is not reusable.

Several attempts were made to produce a chamber which met all the above requirements. Shot L001 used a Swagelok VCO face seal as a chamber. This was an expensive option, and the part swelled due to the dynamic pressure generated during the launch cycle. Shots L002 to L006 used a simple two-flange chamber: one flange is brazed to the launch tube and the other to the pump tube. The flanges are then bolted together with the o-ring and diaphragm in between. This option was less expensive, but the flanges came apart during launch, resulting in loss of driver gas from the chamber.

For shots L007 to L009, the chamber consisted of a thick sleeve screwed onto a threaded rod: the pump tube is brazed to the sleeve and the launch tube to the threaded rod. A nut is welded onto the sleeve which allows it to be screwed onto the threaded rod. The weld did not resist well to the high pressures and cracked during launch, once again resulting in loss of driver gas. Moreover, the o-ring did not seal the fill pressure properly since it was being warped when tightening the rod and sleeve together during assembly.

This led to a three-piece chamber design: the pump tube is brazed onto a threaded piece called the pump tube insert, and the launch tube is brazed to a similar but unthreaded piece called the launch tube insert. The third piece is a 
threaded sleeve, called the chamber sleeve. The launch tube insert is inserted into the chamber sleeve, the o-ring and diaphragm are put into place, and the pump tube insert is screwed into the sleeve until it presses tightly against the launch tube insert. This type of chamber permits the chamber to be assembled without warping the o-ring since there is no rotational motion between the pump tube insert and the launch tube insert. The pump tube insert can also be made with a nipple which fits into the launch tube insert. This causes both pieces to seal against each other under high pressures. This type of chamber performed well and was used for all remaining launcher experiments. However, it was found that the pump tube breaks off the chamber during launch, and sometimes is completely blown out of the pump tube insert: This allows the high-pressure driver gas to expand out the back of the chamber rather than into the launch tube. A breech cone was then added to the pump tube insert. The purpose of this cone is to collapse onto itself and seal the chamber, effectively forming the breech of the launcher. The breech cone was used for all launcher shots starting from L015. As discussed below, it is not clear how effectively it sealed the driver gas into the chamber. A small recess can also be made into the nipple of the pump tube insert. This allows the pump tube to be flared into the insert in order to prevent it from being blown out of the insert upon launch. See Appendix B for detailed part drawings of the final version of the chamber as well as a sketch of the assembled chamber.

\subsubsection{Launch Tube}

The launch tube needs to be straight and rigid, and the inner wall needs to be smooth as possible. Stainless steel tubing was used until shot L007. Shots L008 onward used chrome-molybdenum mechanical tubing. Launch tubes for shots L020 onward were honed with a silicon carbide cylindrical ball hone to ensure a smooth, 
polished inner surface. Brazing the launch tube caused swelling of the inner wall of the tube. This led to problems when inserting the projectile into the launch tube. For shots L016 onward, the launch tube was threaded into the launch tube insert rather than brazed. For many of the higher pressure shots, the launch tube failed close to the chamber. An extra sleeve was added over the launch tube to help it contain the pressure as the projectile starts accelerating. If the projectile was initially positioned too near the diaphragm, the rupture of the diaphragm could cause damage to the projectile. This can be prevented by leaving a distance of about three projectile diameters between the back of the projectile and the diaphragm, as reported by Watson [17].

\subsubsection{Diagnostics}

Several different projectile velocity measurement methods were experimented with. Shots L001, L004, L005, and L006 used break screens. These are made of two thin sheets of brass foil separated by a thin polyester film. The two sheets are connected to a charged capacitor circuit and placed within the path of the projectile. When the projectile hits the screens, it breaks the polyester film and allows the brass sheets to touch, thus completing the circuit and sending a signal to the oscilloscope. However, it was found that break screens are not reliable, especially for projectiles traveling above $1 \mathrm{~km} / \mathrm{s}$. The screens do not always connect upon impact; it is also not clear that, when they do connect, they record the actual time of arrival of the projectile. Fragments from the impact with the first screen are ejected onto the second screen. Since these could travel faster than the projectile, it was unclear what the second break screen was recording. Finally, this is an intrusive velocity measurement technique since the projectile physically impacts the screens. An unintrusive method is preferable. 
Shots L001 to L003 had self-shorting twisted wire pairs inserted into the launch tube. The reasoning was that these would be shorted by the accelerating projectile. They recorded an unusually high acceleration near the muzzle of the launch tube for shot L001, and they gave signals out of sequence for shot L002. They recorded a velocity of nearly $5 \mathrm{~km} / \mathrm{s}$ for shot L003, which is above the expected projectile velocity and is roughly the speed of sound in steel. Since it was unclear what was being recorded by each twisted pair, this technique was found to be unreliable.

Shots L004 to L020 used lasers to record projectile velocity. A laser beam was expanded into a sheet using a cylindrical lens and directed into a second cylindrical lens, which focused the beam into a photodiode. When the projectile cut the laser sheet, the photodiode recorded a drop in light intensity, which can be seen on the oscilloscope. Very accurate aim was required in order for the projectile to cut through at least two laser sheets; four lasers were used for shots L007 onward for accurate velocity measurement. Using the equipment available at the time, this technique was found to work well for velocities up to about $1.5 \mathrm{~km} / \mathrm{s}$, beyond which it was difficult to determine a definite velocity based on the photodiode signals. Also, the projectile was often accompanied by a luminous muzzle blast, which could saturate the photodiodes.

An array of shock pins (Dynasen CA-1135) placed in the windowed chamber (see figure 5-2) served as a secondary velocity measurement diagnostic for shots L011 onward. While these shock pins are intended for measurements of shock waves in solids, it was found that they are sufficiently sensitive to record the bow shock wave resulting from the projectile in air. This array was refined from shot to shot. In the final version (used in shots L015 onward), two rows of three shock pins were glued to a piece of medium-density fiberboard. If the projectile is traveling parallel to the 
array, the bow shock speed corresponds to the bow shock speed. Recorded velocities agreed well with measurements made both with the laser apparatus described above and with high-speed framing camera measurements described below. Although measurements made with this array are sensitive to the projectile trajectory and the muzzle blast which often accompanied the projectile, it is a good secondary velocity measurement diagnostic. A three-dimensional array would be preferable so that the projectile trajectory could be reconstructed in three-dimensions and the true projectile velocity obtained. This diagnostic

The most reliable projectile velocity measurement method was found to be highspeed photography. A Cooke HSFC Pro high-speed framing camera was used to obtain photographs of the in-flight projectile for shots L015 onward. The camera has four different sets of intensifiers and CCDs; each set can record two frames. The camera can therefore record up to eight frames: the first four are each taken by a separate intensifier and CCD, the second four are the second images taken with each intensifier and CCD. The second set of frames often suffer from a "burn" on the intensifier, so that bright objects in the first four frames appear in second four frames. The projectile has to be captured in at least two frames in order to make a velocity measurement. This means the camera has to be timed correctly, which implies the approximate speed of the projectile has to be known. The camera was triggered by contact gauges placed on the muzzle brake (described below). Contact gauges are similar to break screens, but here the two sheets of brass foil are separated by a sheet of two-sided tape which has a hole in the center. Applying a small pressure on a contact gauge will cause it to short. By placing the contact gauges on the muzzle brake they are shorted by either the bow shock from the projectile or the muzzle blast. Note that the camera uses a strobe which saturates the photodiodes used with 
the laser apparatus, rendering them useless. Moreover, using lasers obstructs much of the windowed chamber. The camera and the lasers are therefore not compatible with each other and only one of the two should be used.

On the shots where the high-speed framing camera was initially used, a jet of bright gas could be seen in the windowed chamber. This jet could either be the gas initially in the launch tube which is shocked and pushed out of the launch tube by the projectile or the driver gas exiting the launch tube behind the projectile. It is impossible to determine the location of the projectile if it is inside the bright jet when the frames are recorded. Moving the launcher further away from the windowed chamber gives time for the jet to decelerate due to drag and for the projectile to emerge from it. A "muzzle brake" was also added to launchers L017 onward. This "muzzle brake" is intended to strip away as much gas as possible from the projectile to delay the jet and to allow the projectile to emerge from the jet earlier. It consists of a metal disc placed about $4 \mathrm{~cm}$ from the muzzle. The metal disc has a hole in its center which has a diameter about $1.5 \mathrm{~mm}$ larger than that of the projectile.

For the higher projectile velocities recorded, the high-speed images obtained with the camera showed a luminous trail in the wake of the projectile. This was probably caused by the projectile ablating as it traveled in the ambient atmosphere. The windowed chamber and launch tube of the launcher were flushed with helium for shots L023 onward. This eliminated the luminous projectile ablation. The setup for this is shown in figure $5-2$.

\subsection{Experimental Launcher Performance}

The final launcher design used is represented in figure 5-1. The measurements are reported in section 4.4 and the detail drawings are given in Appendix B. The setup of a launcher experiment, including testing facilities and diagnostics are shown 
in figure 5-2. The launcher was placed on a work table within the blast chamber. The projectile was fired into a windowed chamber attached to the blast chamber. A baffle was placed at the opening of the windowed chamber to limit the amount of debris projected into it. The launcher aim was set by aligning it with a laser level positioned in front of the windowed chamber. The high-speed framing camera was used to obtain photographs of the in-flight projectile within the windowed chamber. A strobe was used with the camera; it was positioned at an angle behind the windowed chamber such that the lighting element would not appear on the photographs. The camera was triggered by contact gauges on the muzzle brake of the launcher. The array of shock pins was placed inside the windowed chamber and recorded the bow shock of the projectile. For shots where the windowed chamber and the launch tube were flushed with helium, a flexible plastic tube was extended from the baffle over the chamber of the launcher; the windowed chamber and flexible tube were then flushed with helium.

Figure 5-3 shows a launcher before and after a shot. Note that even if the tamper was made thick enough to survive pump tube expansion, the post-detonation pressure was still many orders of magnitude greater than its bursting pressure; the tamper was therefore always destroyed. The pump tube was always recovered in one or several pieces separate from the chamber. Even if the chamber did not fail during launch, it was not reusable since the high internal pressures it experienced caused it to expand internally, sealing all pieces together and making it impossible to take it apart.

\subsubsection{Compression Ratio Variation}

The first systematic series of launcher experiments (shots L017 to L023) verified the relationship between projectile muzzle velocity and compression ratio, the details 


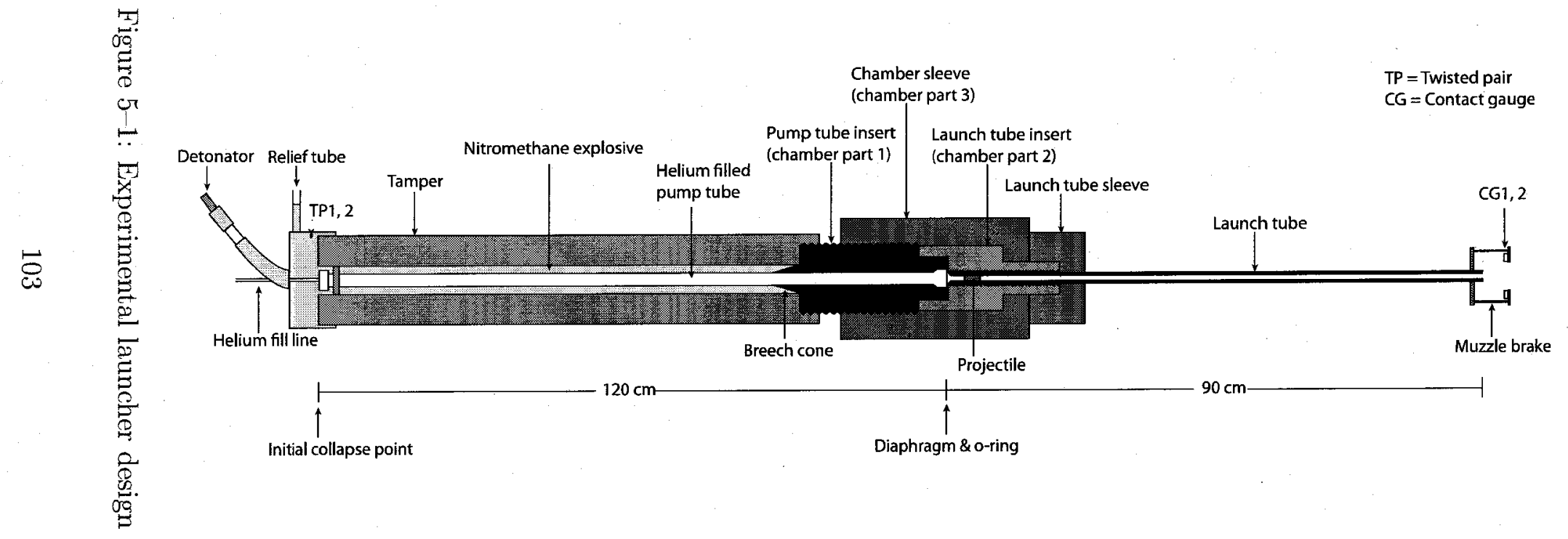




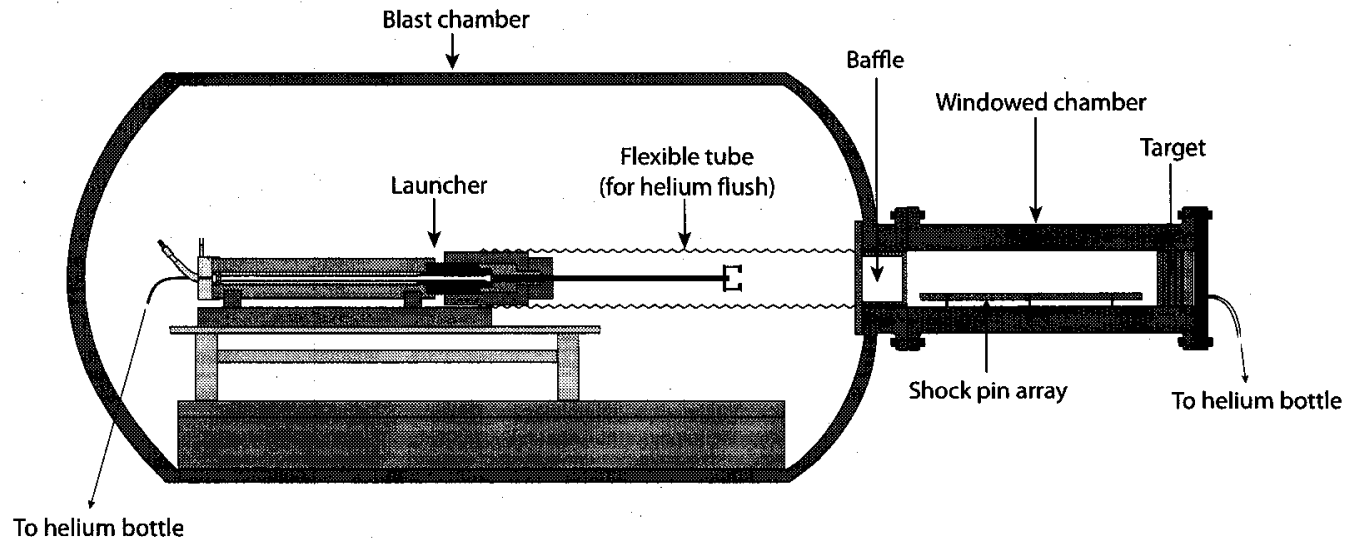

Figure 5-2: Experimental launcher setup, showing facilities and diagnostics

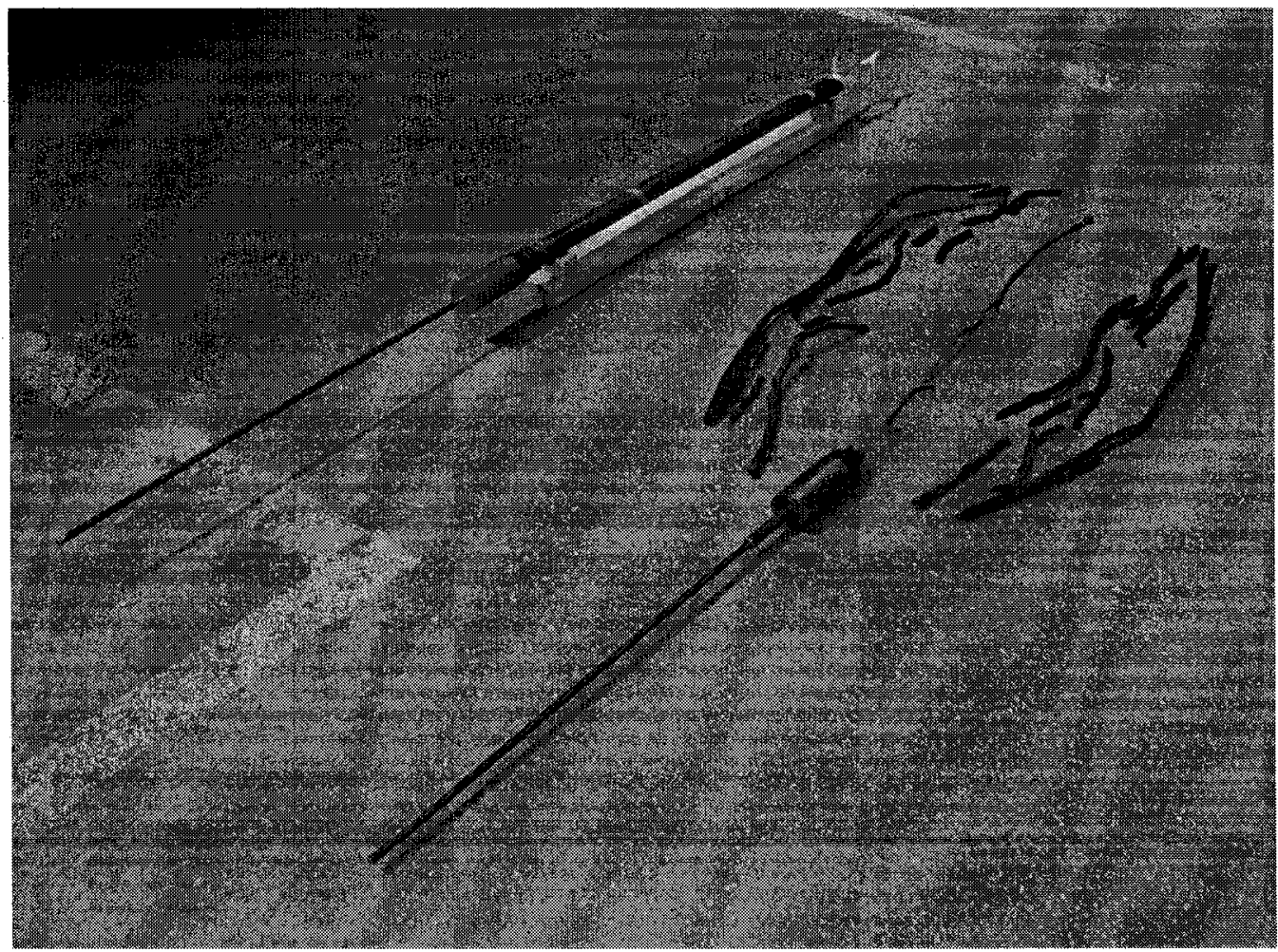

Figure 5-3: Picture of launchers, before and after firing 
of which can be found in table 5-3. These experiments were done at an initial helium fill pressure of $20 \mathrm{~atm}$. The experimental data is shown in figure 5-4. The symbols used for velocity represent the velocity as measured by analysis of the highspeed images. The error bars represent the possible range of projectile velocities as determined from both the error in analyzing images and in the recorded shock pin signals. Also shown are the calculated launcher performance for both a sealed chamber and a venting chamber as predicted by the Euler code, as discussed in Chapter 4 .

The experimental launcher performance is much closer to the venting chamber case than the sealed chamber case. The projectile velocity is around $3 \mathrm{~km} / \mathrm{s}$ and does not increase significantly for compression ratios less than 10 . Therefore, according to these results, the breech cone is not effective at containing the high-pressure driver gas within the chamber.

In the experimental data, we note an increase in projectile velocity for a compression ratio of 11.5. This is consistent with the discussion in section 4.4, where we see that for compression ratios above 10 , the shock hits the projectile a second time, which leads to higher projectile velocities. However, the launcher performance then decreases as we increase the compression ratio further. This is probably due to the reflected shock exiting the chamber, causing the pump tube to burst and disrupting the incoming implosion pinch. Increasing the compression ratio further results in occasional loss of the projectile, as seen in shot L019. Based on these results, a compression ratio of 11.5 is optimal for the present launcher.

\subsubsection{Initial Fill Pressure Variation}

For the optimal compression ratio found above, we can now increase the initial fill pressure until we see a decrease in launcher performance. Experiments were done 
Table 5-3: Summary of launcher experiments testing compression ratio variation. All shots done at 20 atm initial fill pressure.

\begin{tabular}{|c|c|c|c|c|}
\hline $\begin{array}{l}\text { Shot } \\
\text { No. }\end{array}$ & $\mathrm{CR}$ & $\begin{array}{l}\text { Projectile } \\
\text { Velocity } \\
(\mathrm{km} / \mathrm{s})\end{array}$ & $\begin{array}{l}\text { Projectile } \\
\text { mass } \\
(\mathrm{g})\end{array}$ & Comments \\
\hline L015 & 11.47 & 3.27 & 0.252 & $\begin{array}{l}\text { The pump tube was damaged when pressing it into } \\
\text { the chamber insert. The launch tube swelled when } \\
\text { welding it to the chamber. An undersized projectile } \\
\text { was used. No reliable signals from lasers. Pictures } \\
\text { lost while attempting to save them; camera operator } \\
\text { reported seeing intact projectile on 2nd frame and a } \\
\text { bright jet on all frames. }\end{array}$ \\
\hline L016 & 6.17 & - & 0.289 & $\begin{array}{l}\text { The launch tube was threaded onto the launch tube } \\
\text { insert. The launch tube failed. The projectile was } \\
\text { not recovered intact; fragments were found on the } \\
\text { impact plate. No reliable signals from lasers. No } \\
\text { projectile was visible on the pictures, only a bright } \\
\text { jet. }\end{array}$ \\
\hline L017 & 8.92 & 3.3 & 0.290 & $\begin{array}{l}\text { A muzzle break was added to help prevent the muzzle } \\
\text { jet. The projectile was recovered intact. No reliable } \\
\text { signals from lasers. The projectile was visible on two } \\
\text { pictures. The launch tube sleeve swelled. }\end{array}$ \\
\hline L018 & 11.47 & 4.1 & 0.290 & $\begin{array}{l}\text { The launch tube failed despite the sleeve. The pro- } \\
\text { jectile was not recovered; fragments were found on } \\
\text { the impact plate. No reliable signals from lasers. } \\
\text { The projectile was visible in the second frame; for } \\
\text { the camera settings used, this means it must have } \\
\text { been traveling upwards of } 4.1 \mathrm{~km} / \mathrm{s} \text {. }\end{array}$ \\
\hline L019 & 16.09 & 3.2 & 0.288 & $\begin{array}{l}\text { The projectile was not recovered, nothing was found } \\
\text { on the impact plate. No reliable signals from lasers. } \\
\text { Pictures indicate the projectile is decelerating very } \\
\text { fast. Suspect projectile was damaged or destroyed } \\
\text { upon launch. }\end{array}$ \\
\hline L020 & 7.29 & 3.45 & 0.292 & No reliable signals from lasers. \\
\hline $\mathrm{L} 021$ & 6.17 & 3.2 & 0.325 & $\begin{array}{l}\text { The lasers were not used since they are not compat- } \\
\text { ible with the strobe. This allows the entire window } \\
\text { to be in the field of view of the camera. }\end{array}$ \\
\hline L022 & 13.38 & 3.9 & 0.290 & $\begin{array}{l}\text { The windowed chamber and launch tube were flushed } \\
\text { with helium to prevent ablation of the projectile. } \\
\text { Data from the shock pins was inconclusive. }\end{array}$ \\
\hline L023 & 16.09 & 3.76 & 0.283 & $\begin{array}{l}\text { The windowed chamber and launch tube were flushed } \\
\text { with helium. The breech cone did not perform well. } \\
\text { The shock pin signals were not as steep as usual. }\end{array}$ \\
\hline
\end{tabular}




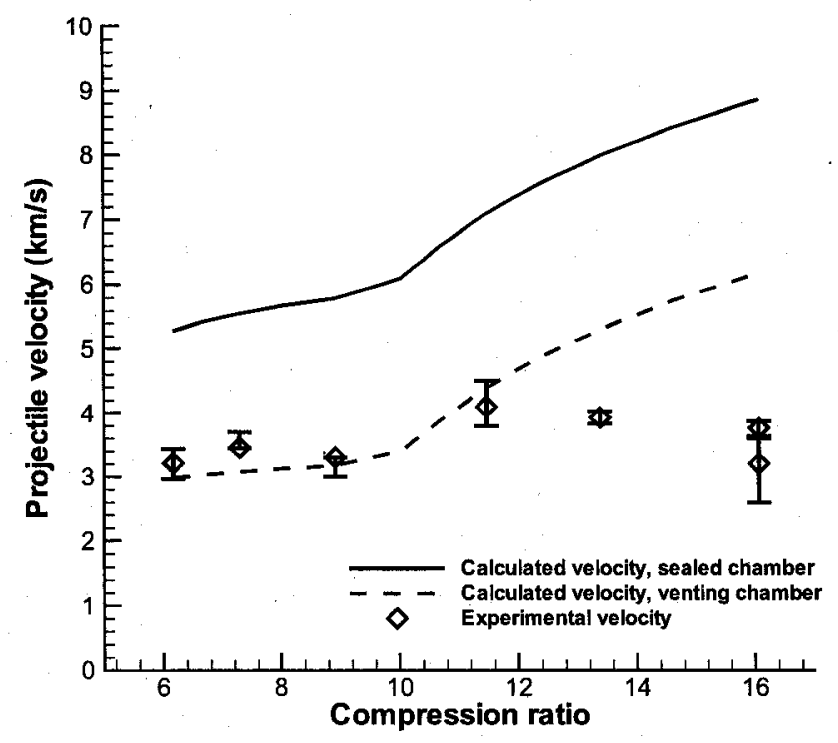

Figure 5-4: Projectile velocity for compression ratios from 6 to 16 for an initial fill pressure of $20 \mathrm{~atm}$

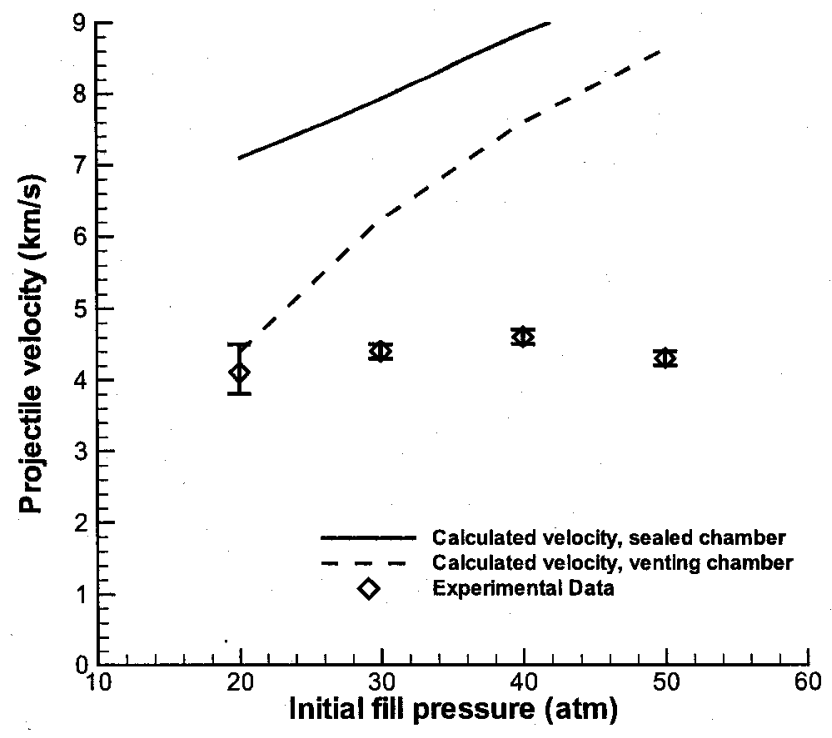

Figure 5-5: Projectile velocity for initial fill pressures from 20 to $50 \mathrm{~atm}$ for a compression ratio of 11.5 
Table 5-4: Summary of launcher experiments testing initial fill pressure variation. All shots done at a compression ratio of 11.47 .

\begin{tabular}{|c|c|c|c|l|}
\hline $\begin{array}{c}\text { Shot } \\
\text { No. }\end{array}$ & $\begin{array}{c}\text { Fill } \\
\text { Pressure } \\
(\mathrm{atm})\end{array}$ & $\begin{array}{c}\text { Projectile } \\
\text { Velocity } \\
(\mathrm{km} / \mathrm{s})\end{array}$ & $\begin{array}{c}\text { Projectile } \\
\text { mass } \\
(\mathrm{g})\end{array}$ & \multicolumn{1}{|c|}{ Comments } \\
\hline L024 & 30 & 4.4 & 0.287 & $\begin{array}{l}\text { The breech cone did not perform as well as for the } \\
\text { 20 atm shot for the same compression ratio (L018). }\end{array}$ \\
\hline L025 & 40 & 4.7 & 0.280 & $\begin{array}{l}\text { The pictures were lost due to a failure of the strobe, } \\
\text { but the shock pins gave reliable signals. The breech } \\
\text { cone did not perform as well as for both the 20 atm } \\
\text { and 30 atm shots for the same compression ratio } \\
\text { (L018 and L024). }\end{array}$ \\
\hline L026 & 50 & 4.25 & 0.290 & The breech cone was destroyed. \\
\hline
\end{tabular}

where the initial fill pressure was increased up to 50 atm for this compression ratio (shots L024 to L026). The results are shown in figure 5-5 and the details of the individual shots can be found in table 5-4. Also shown are the calculated launcher performance for both a sealed chamber and a venting chamber from the Euler code.

For an initial fill pressure of 20 atm, the experimental data agrees well with the venting chamber solution from the Euler code. However, as we increase the fill pressure, the experimental performance falls below even the venting chamber simulation results. The fact that the performance is even less than a complete absence of sealing at the back of the breech might be attributed to radial expansion of the breech as well, resulting in an addition mechanism in loss of driver pressure. Another possible explanation would be the observed decrease in driver performance with increasing initial fill pressure. Nevertheless, launcher performance does increase until a fill pressure of $40 \mathrm{~atm}$. Beyond this point, launcher performance decreases with increasing fill pressure. 


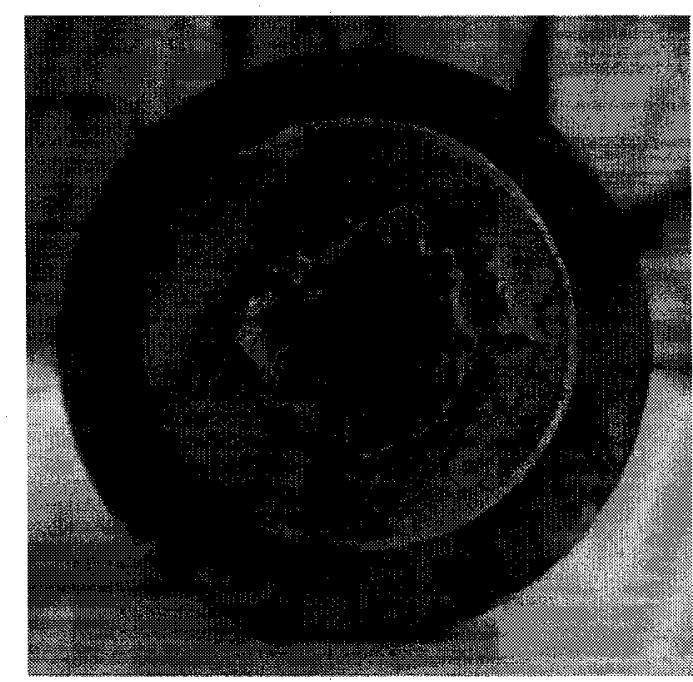

(a) $20 \mathrm{~atm}$ (L018)

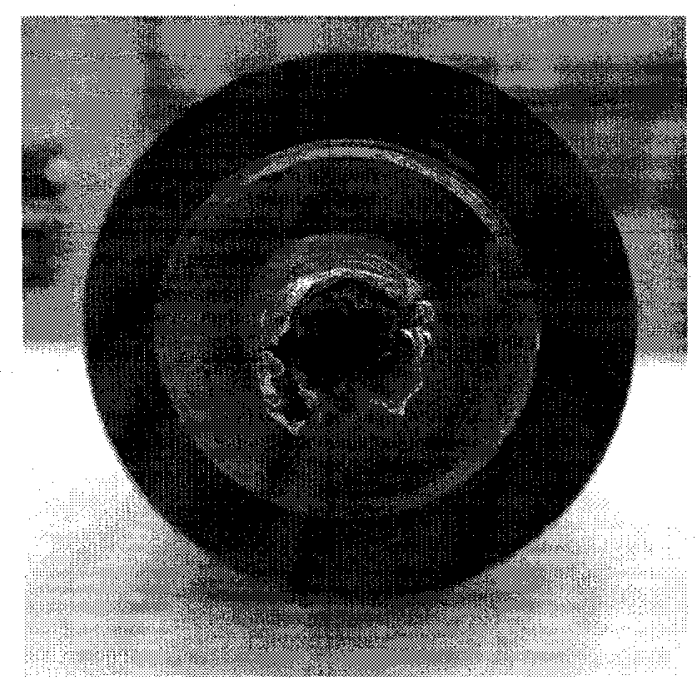

(c) $40 \mathrm{~atm}(\mathrm{~L} 025)$

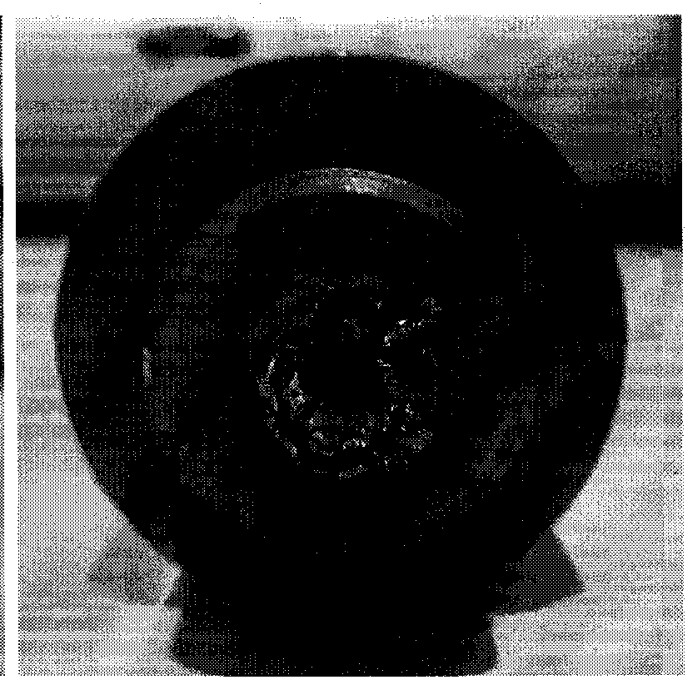

(b) $30 \mathrm{~atm}$ (L024)

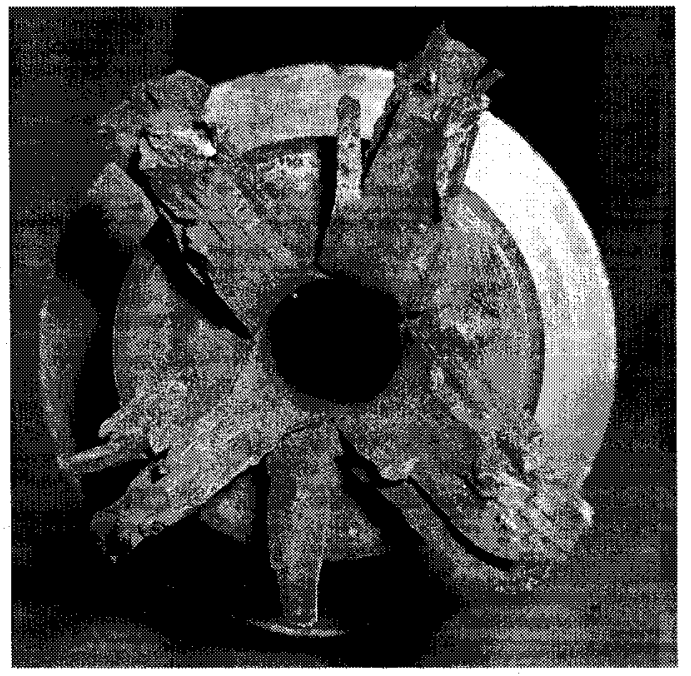

(d) $50 \mathrm{~atm}(\mathrm{~L} 026)$

Figure 5-6: Pictures of breech cones after firing for various initial fill pressures. All shots displayed are for a CR of 11.47. 


\section{CHAPTER 6 \\ Conclusions}

The behaviour of the linear explosive driver has been studied theoretically and experimentally. A simple analytic model was used to simulate pump tube expansion and its effect on the precursor shock. Through comparison of calculated and experimental results, it was confirmed that this is the main non-ideal effect affecting driver performance. Increasing the initial fill pressure of the driver gas leads to increased expansion of the pump tube which decreases the precursor shock velocity and the standoff distance between the shock and the virtual piston. The developed model indicates that surrounding the explosives with a tamper limits pump tube expansion and prevents or delays pump tube failure. However, experimental results did not indicate a significant difference in performance between tamped and untamped drivers. Using a tamper does nonetheless reduce the amount of explosives needed to properly collapse a given tube. Detonation velocities recorded in tamped driver experiments were above the usual detonation velocities of the explosives used. This is presumably due to the compression of the explosives between the tamper and the expanding pump tube, although the increase in detonation velocity is greater than predicted by theory for nitromethane explosive. The performance of the larger pump tubes experimented with surpassed that of the smaller tubes. Experiments also showed that the thinner pump tubes perform better.

The performance of the linear explosive driver as a hypervelocity launcher was then studied theoretically and experimentally. A quasi-one-dimensional Eulerian solver was used to calculate an upper bound on the capabilities of the launcher for 
various conditions. An important factor influencing performance was found to be the compression ratio of the launcher. Increasing the compression ratio increases both the frequency and the strength of the interactions of the shock with the projectile, increasing final projectile velocity. Simulations also show that, if the chamber is not sealed and vents driver gas, the performance of the launcher increases significantly only for compression ratios above 10 .

Launcher experiments were performed. It was found that a three-piece chamber design with a nipple performed well. High speed photography was found to be the most reliable way to measure projectile velocity; a shock pin array measuring the bow shock from the projectile served as an acceptable secondary diagnostic. Comparison of experimental launcher performance with simulated results indicates that the breech cone is not properly sealing the chamber. A maximum launcher performance was observed for a compression ratio of 11.5 and an initial fill pressure of $40 \mathrm{~atm}$. For these values, an intact $0.3 \mathrm{~g}$ projectile was successfully launched at $4.7 \mathrm{~km} / \mathrm{s}$. Increasing these values further results in a decrease in performance due to . damage to the launcher and increased pump tube expansion or pump tube failure; projectile integrity could also be compromised. 


\section{Appendix A - Expanding Tube Model Algorithm}

Define the following:

Dimensions; properties of the material, gas and explosive; pressure of the driver gas; distance of the examined cross section from the initial piston location; velocity of the piston; time-step size.

Calculate the standoff and volume of shocked gas in the rigid tube case.

Solve the differential equations:

Tube expansion:

RK4 step for pump tube dynamics (equation 2.3):

$$
\ddot{r}_{p t}=\left[\frac{\left(p_{\text {internal }}-p_{\text {external }}\left(r_{p t}, \dot{r}_{p t}, r_{o t}, \dot{r}_{o t}\right)\right) r}{r_{0} \tau_{0} \rho}-\frac{\sigma_{h}(r, \dot{r})}{r \rho}\right]_{p t}
$$

RK4 step for outer tube dynamics (equation 2.3):

$$
\ddot{r}_{o t}=\left[\frac{p_{\text {internal }}\left(r_{p t}, \dot{r}_{p t}, r_{o t}, \dot{r}_{o t}\right) r}{r_{0} \tau_{0} \rho}-\frac{\sigma_{h}(r)}{r \rho}\right]_{o t}
$$

\section{Pressure in the explosive layer:}

Pump Tube:

Pressure wave sent out due to change in wall speed (equation 2.8):

$$
\Delta p=\rho_{0} c_{0} \Delta u=\rho_{0} c_{0}(u(t)-u(t-\Delta t))
$$

Find the communication time $t_{c}$ iteratively:

$$
t_{c}=\frac{r_{o t}\left(t-t_{c}\right)-r_{p t}(t)}{c}
$$


Based on $t_{c}$, determine how many reflected waves hit the pump tube since the last time-step (find $\sum \Delta p_{\text {reflected }}$ ).

Calculate the pressure on the pump tube (equation 2.9):

$$
p_{\text {external }}(t)=p(t-\Delta t)+\Delta p(t)+2 \sum \Delta p_{\text {reflected }}
$$

Calculate the total strength of the pressure wave sent out from the pump tube at this time-step (equation 2.10):

$$
[\Delta p(t)]_{t o t}=\Delta p(t)+\sum \Delta p_{\text {reflected }}
$$

Outer Tube:

Pressure wave sent out due to change in wall speed (equation 2.8):

$$
\Delta p=\rho_{0} c_{0} \Delta u=\rho_{0} c_{0}(u(t)-u(t-\Delta t))
$$

Find the communication time $t_{c}$ iteratively:

$$
t_{c}=\frac{r_{o t}(t)-r_{p t}\left(t-t_{c}\right)}{c}
$$

Based on $t_{c}$, determine how many reflected waves hit the outer tube since the last time-step (find $\sum \Delta p_{\text {reflected }}$ ).

Calculate the pressure on the outer tube (equation 2.9):

$$
p_{\text {internal }}(t)=p(t-\Delta t)+\Delta p(t)+2 \sum \Delta p_{\text {reflected }}
$$

Calculate the total strength of the pressure wave sent out from the outer tube at this time-step (equation 2.10):

$$
[\Delta p(t)]_{t o t}=\Delta p(t)+\sum \Delta p_{\text {reflected }}
$$




\section{Stress-strain relations:}

Pump Tube:

Strain in the pump tube: $\varepsilon(t)=1-\frac{r(t)}{r_{0}}$

Total undergone deformation: $\varepsilon_{f}(t)=\varepsilon_{f}(t-\Delta t)+|\varepsilon(t)-\varepsilon(t-\Delta t)|$

Strain rate: $\dot{\varepsilon}^{*}=\dot{\varepsilon} / \dot{\varepsilon}_{0}$

Temperature change due to plastic heating (equation 2.5):

$$
\Delta T=\frac{\beta}{\rho C_{p}} \int_{0}^{\varepsilon_{f}} \sigma d \varepsilon
$$

Homologous temperature: $T^{*}=\frac{T-T_{\text {room }}}{T_{\text {melt }}-T_{\text {room }}}$

Apply Johnson-Cook strength model (equation 2.4):

$$
\sigma_{h}(t)=\left[A+B \varepsilon^{\eta}\right]\left[1+C \ln \dot{\varepsilon}^{*}\right]\left[1-T^{* m}\right]
$$

Outer Tube (if the outer tube is a tamper; if not, stress is zero):

Strain in the tamper: $\varepsilon(t)=1-\frac{r(t)}{r_{0}}$

Stress in the tamper:

Elastic regime: $\sigma_{h}(t)=E \varepsilon(t)$

Plastic regime: $\sigma_{h}(t)=\sigma_{y}$

\section{Standoff Calculation:}

Convert time to distance: $\Delta x=U_{s} \Delta t$

Calculate the volume of gas shocked during the current time-step, and add it to the total volume of shocked gas:

$$
V o l(t)=\operatorname{Vol}(t-\Delta t)+\pi\left(r_{p t}(t)\right)^{2} \Delta x
$$


Update the standoff: $x(t)=x(t-\Delta t)+\Delta x$

Once $V$ ol reaches the value of the rigid tube case, the piston has reached the cross section we are looking at. 


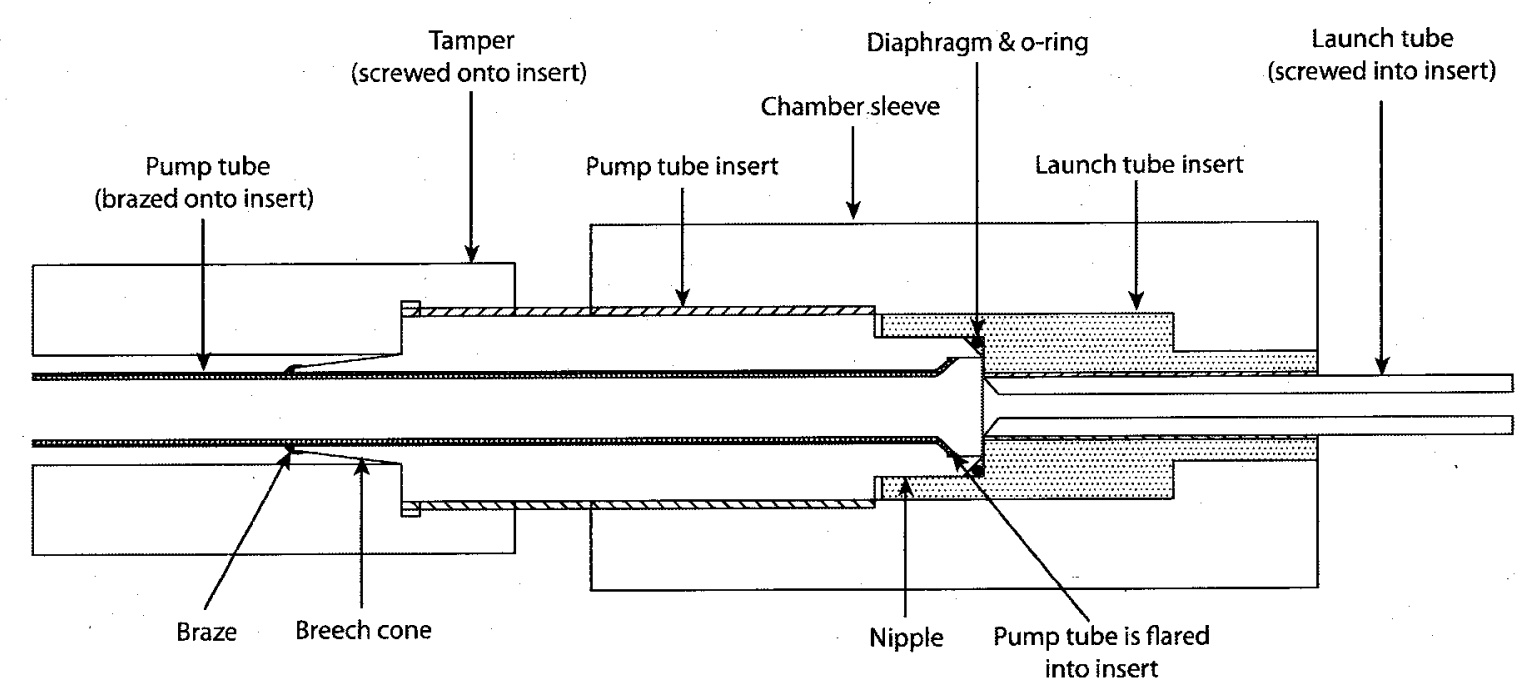




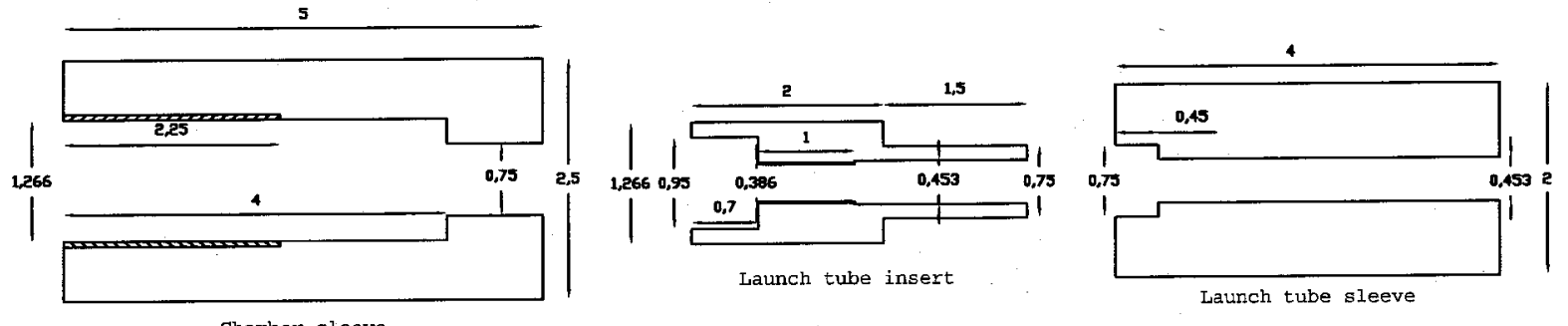

Chamber sleeve
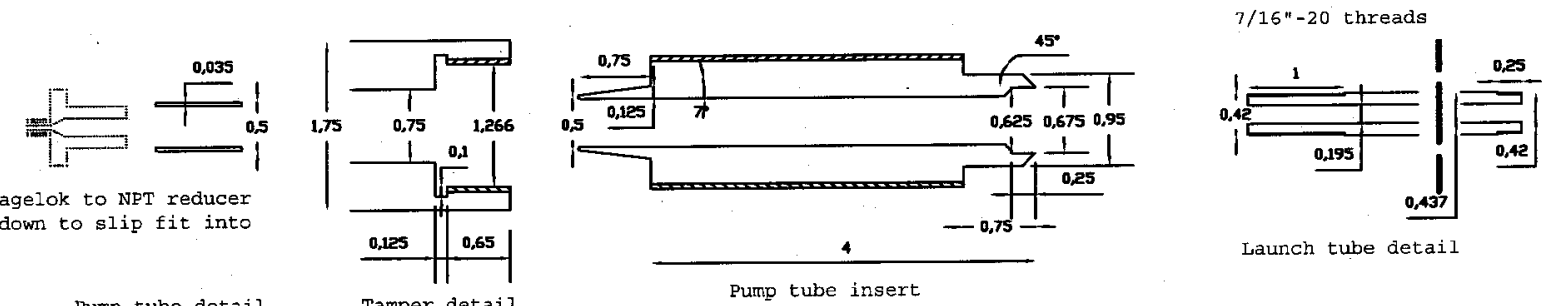

Plug is modified 1/8" swagelok to NPT reducer with NPT thre
pump tube.

Pump tube detail

Tamper detail

Pump tube insert

Launch tube aetail

Tap 1.5" disk center with 7/16"-20 threads.

Tap both hole arrays with 10-32 and attach

with 2" screws.

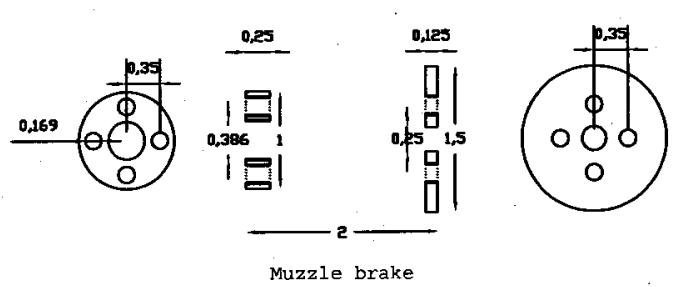

Ensure final machined dimensions permit good part it according to assembly.

Tamper is $1.75^{\prime \prime}$ oD $-0.75 "$ ID mechanical tubing, $44^{4}$ in length. Optionally cut shorter for longer pump tube inserts (lower compression ratios).

Pump tube is 0.5 " OD, 0.035 " wall, 47 " in length seamless stainless steel tubing.

Launch tube is $0.437 "$ OD (nominal), $0.125 "$ wall (nominal) chrome-moly tubing. 
References

[1] D. W. Baum. "Development of explosively driven launcher for meteoroid studies." NASA CR-2143, 1973.

[2] G. Birkhoff, D. P. MacDougall, E. M. Pugh, and G. Taylor. "Explosives with lined cavities." Journal of Applied Physics, vol. 19, June 1948, pp. 563-582.

[3] J. Carleone and R. Stefan. "A model for the collapse of explosively driven liners in spinning warheads." Propellants, Explosives, Pyrotechnics, vol. 18, 1993, pp. 299-306.

[4] J. K. Crosby and S. Gill. "Feasibility study of an explosive gun." NASA CR-709, 1967.

[5] G. R. Johnson and W. H. Cook. "A constitutive model and data for metals subjected to large strains, high strain rates and high temperatures." In Proceedings of the 7th International Symposium on Ballistics. The Hague, The Netherlands, April 1983, pp. 541-547.

[6] S. A. Kinelorskii. "Collapse of metallic pipes under the action of an explosive." Combustion, Explosion and Shock Waves (Fizika Goreniya $i$ Vzrya), vol. 16, no. 6,1980 , pp. $73-79$.

[7] S. A. Kinelovskii. "Collapse of metal tubes under the explosive loading at a finite thickness of an explosive charge." Combustion, Explosion and Shock Waves (Fizika Goreniya i Vzrya), vol. 10, no. 2, 1983, pp. 110-115.

[8] W. S. Koski, F. A. Lucy, R. G. Shreffler, and F. J. Willig. "Fast jets from collapsing cylinders." Journal of Applied Physics, vol. 23, no. 12, December 1952, pp. 1300-1305.

[9] H. Mirels. "Shock tube test time limitations due to turbulent-wall boudary layer." AIAA Journal, vol. 2, no. 1, January 1964, pp. 84-93.

[10] E. T. Moore. "Explosive hypervelocity launchers." NASA CR-982, 1968.

[11] O. E. Petel, V. Tanguay, A. J. Higgins, A. C. Yoshinaka, and F. Zhang. "Detonation propagation in shock-compressed liquid explosives." In Shock Compression of Condensed Matter - 2003: Proceedings of the Conference of the American 
Physical Society Topical Group on Shock Compression of Condensed Matter. AIP, 2004, pp. 883-886.

[12] N. F. Ponchaut. "Euler solver for rapidly deforming domains." unpublished, July 2006. Contact author at nicolas@alumni.caltech.edu.

[13] G. Rudinger. Nonsteady Duct Flow Wave Diagram Analysis. Dover Publications, Inc., 1969.

[14] A. E. Seigel. "The theory of high speed guns." AGARDograph 91, May 1965.

[15] E. F. Toro. Riemann Solvers and Numerical Methods for Fluid Dynamics - A Practical Introduction,. Springer-Verlag, 2nd edn., 2006.

[16] H. F. Waldron, E. T. Moore Jr., G. B. Steel, and C. S. Godfrey. "A mechanism for the conversion of the chemical energy of explosives to the kinetic and internal energy of a gas." AIAA 5th Aerospace Sciences Meeting, January 1967. AIAA paper number $67-178$.

[17] J. D. Watson. "High-velocity explosively driven guns." NASA CR-1533, 1970.

[18] J. M. Winey, G. E. Duvall, M. D. Knudson, and Y. M. Gupta. "Equation of state and temperature measurements for shocked nitromethane." Journal of Chemical Physics, vol. 113, 2000, pp. 7492-7501. 